\title{
Diagrammatic description of $c$-vectors and $d$-vectors of cluster algebras of finite type
}

To the memory of Andrei Zelevinsky

\author{
Tomoki Nakanishi \\ Graduate School of Mathematics \\ Nagoya University \\ Chikusa-ku, Nagoya, Japan \\ nakanisi@math.nagoya-u.ac.jp
}

\author{
Salvatore Stella* \\ Department of Mathematics \\ North Carolina State University \\ Raleigh, NC, USA \\ sstella@ncsu.edu
}

Submitted: Jan 31, 2013; Accepted: Dec 23, 2013; Published: Jan 12, 2014

Mathematics Subject Classifications: 13F60

\begin{abstract}
We provide an explicit Dynkin diagrammatic description of the $c$-vectors and the $d$-vectors (the denominator vectors) of any cluster algebra of finite type with principal coefficients and any initial exchange matrix. We use the surface realization of cluster algebras for types $A_{n}$ and $D_{n}$, then we apply the folding method to $D_{n+1}$ and $A_{2 n-1}$ to obtain types $B_{n}$ and $C_{n}$. Exceptional types are done by direct inspection with the help of a computer algebra software. We also propose a conjecture on the root property of $c$-vectors for a general cluster algebra.
\end{abstract}

\section{Introduction}

\section{$1.1 \quad$ Background}

For a given skew-symmetrizable integer matrix $B$, let $\mathcal{A}_{\bullet}(B)$ be the cluster algebra with principal coefficients whose initial exchange matrix is $B[26,28]$. Note that $\mathcal{A}_{\bullet}(B)$ depends on $B$ itself (not on its mutation equivalence class) due to the presence of principal coefficients. There are two important families of integer vectors associated with $\mathcal{A}_{\bullet}(B)$ : c-vectors and d-vectors. The former are the column vectors in the bottom half square matrices $(C$-matrices) of the extended exchange matrices of $\mathcal{A} \cdot(B)$. The latter are also called the denominator vectors; they are the tuples of the exponents in the denominators of the Laurent expansions of the cluster variables of $\mathcal{A}_{\bullet}(B)$ in terms of the initial

*Partially supported by A. Zelevinsky's NSF grant DMS-1103813 and Northeastern University 
cluster. An alternative way to introduce them is: $c$-vectors are the tropicalized versions of coefficients ( $y$-variables) and $d$-vectors are the tropicalized version of cluster variables ( $x$-variables), respectively. See Section 2.1 for details.

Fix an indexing set $I$. Following [27], to each skew-symmetrizable matrix $B=\left(b_{i j}\right)_{i, j \in I}$, we assign a symmetrizable matrix $A(B)=\left(a_{i j}\right)_{i, j \in I}$ called the Cartan counterpart of $B$, by setting

$$
a_{i j}= \begin{cases}2 & i=j \\ -\left|b_{i j}\right| & i \neq j .\end{cases}
$$

The matrix $A(B)$ is a symmetrizable (generalized) Cartan matrix in the sense of Kac [34]. It has been partially recognized and proved that, the $c$ - and $d$-vectors of $\mathcal{A}_{\bullet}(B)$ are roots of the root system of the Cartan matrix $A(B)$. When $B$ is skew-symmetric, thanks to Kac's theorem [32], it is enough to prove that the vectors (or their negatives) are identified with the dimension vectors of some indecomposable modules of the path algebra $k Q(B)$ for the quiver $Q(B)$ corresponding to $B$. In fact, this is a common method of proving many known cases. We are going to discuss this subject in more detail in Section 2 .

Cluster algebras of finite type, i.e., the ones with finitely many seeds, form one of the most basic and important classes of cluster algebras [27]. They have been intensively studied in particular in the cases when $B$ is skew-symmetric, i.e. when $\mathcal{A}_{\bullet}(B)$ is of one of the simply-laced types $A_{n}, D_{n}, E_{6}, E_{7}, E_{8}$ according to the classification of [27]. In these cases the cluster-tilted algebra $\Lambda(B)$, introduced in [7] as a certain quotient of the path algebra $k Q(B)$, plays a key role in the study of $\mathcal{A}_{\bullet}(B)[12,13,4,8,6]$. A $c$-vector is said to be positive if it is a nonzero vector and its components are all nonnegative. A $d$-vector is non-initial if it is the $d$-vector of a non-initial cluster variable. It was proved by $[13,7]$ that the set of all the non-initial $d$-vectors of $\mathcal{A}_{\bullet}(B)$ coincides with the set of the dimensions vectors of all the indecomposable $\Lambda(B)$-modules. Moreover, it was recently proved by $[42,40]$ that the set of all the positive $c$-vectors of $\mathcal{A}_{\bullet}(B)$ also coincides with the same set. See Theorems 2.5 and 2.6.

In spite of this beautiful and complete, representation-theoretic description of $c$ - and $d$-vectors for finite type, little is known about their explicit form, except for type $A_{n}$ $[12,44,53]$. The purpose of this paper is to fill this gap and to provide an explicit Dynkin diagrammatic description of the $c$ - and $d$-vectors of cluster algebras of any finite type with any initial exchange matrix.

It is our hope that the lists presented here will be useful for studying cluster algebras, as the appendix of [3] is for studying Lie algebras.

\section{$1.2 \quad$ Main results}

We present here the main results of the paper. Recall that, for a skew-symmetrizable matrix $B$, the cluster algebra $\mathcal{A}_{\bullet}(B)$ is of finite type if and only if $B$ is mutation equivalent to a matrix $B^{\prime}$ whose Cartan counterpart $A\left(B^{\prime}\right)$ is a Cartan matrix of finite type, $A_{n}, B_{n}$, $C_{n}, D_{n}, E_{6}, E_{7}, E_{8}, F_{4}, G_{2}[27]$. We say that such a skew-symmetrizable matrix $B$ is of cluster finite type, and also, more specifically, of cluster type $Z$, according to the type $Z$ 
of $A\left(B^{\prime}\right)$ above. For any skew-symmetrizable matrix $B$ of cluster finite type, we present the Cartan matrix $A(B)$ as a Dynkin diagram $X(B)$ in the usual way following [34]. Note that, in general, $X(B)$ is not a finite type Dynkin diagram.

For each finite type $Z$, we provide the following two lists explicitly:

- the list $\mathcal{X}(Z)$ of the Dynkin diagrams $X(B)$ of all the skew-symmetrizable matrices $B$ of cluster type $Z$ (for each $B$ the vertices of $X(B)$ are naturally identified with elements of $I$ ),

- the list $\mathcal{W}(Z)$ of the "templates" of positive $c$-vectors and non-initial $d$-vectors in the form of weighted Dynkin diagrams, namely, Dynkin diagrams with a positive integer attached to each vertex.

For a pair $X(B) \in \mathcal{X}(Z)$ and $W \in \mathcal{W}(Z)$, an embedding of the diagram part of $W$ into $X(B)$ as a full sub-diagram is denoted by $W \subset X(B)$. Such an embedding is not necessarily unique if it exists; we distinguish them up to isomorphism of $W$. To each embedding $W \subset X(B)$ corresponds an integer vector $v=\left(v_{i}\right)_{i \in I}$ : its $i$-th component $v_{i}$ is the weight of $W$ at $i$.

For each skew-symmetrizable matrix $B$ of cluster type $Z$, let us introduce the sets

$$
\begin{aligned}
\mathcal{V}(B) & :=\{W \subset X(B) \mid W \in \mathcal{W}(Z)\}, \\
\mathcal{C}(B) & :=\left\{\text { all } c \text {-vectors of } \mathcal{A}_{\bullet}(B)\right\} \\
\mathcal{C}_{+}(B) & :=\left\{\text { all positive } c \text {-vectors of } \mathcal{A}_{\bullet}(B)\right\} \\
\mathcal{D}(B) & :=\left\{\text { all non-initial } d \text {-vectors of } \mathcal{A}_{\bullet}(B)\right\} .
\end{aligned}
$$

For finite type cluster algebras, it turns out that

$$
\mathcal{C}(B)=\mathcal{C}_{+}(B) \sqcup\left(-\mathcal{C}_{+}(B)\right),
$$

therefore, we can concentrate on $\mathcal{C}_{+}(B)$. Our main result is stated as follows.

Theorem 1.1. Let $B$ be any skew-symmetrizable matrix of cluster finite type. Then, the sets $\mathcal{C}_{+}(B), \mathcal{D}(B)$, and $\mathcal{V}(B)$ coincide.

Let us illustrate the content of Theorem 1.1 by mean of a baby example; the reader can find slightly bigger examples at the end of Section 3.

Example 1.2. The matrix

$$
B=\left(\begin{array}{ccc}
0 & 1 & -1 \\
-1 & 0 & 1 \\
1 & -1 & 0
\end{array}\right)
$$

is of cluster type $A_{3}$ and the Dynkin diagram $X(B)$ corresponding to it is

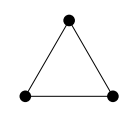

There are precisely three templates in $\mathcal{W}\left(A_{3}\right)$ : 
The first and second of them can be embedded as full sub-diagram into $X(B)$ in three different ways each while the third one can't be embedded into $X(B)$. We get therefore 6 vectors:

$$
\mathcal{V}(B)=\{(1,0,0),(0,1,0),(0,0,1),(1,1,0),(1,0,1),(0,1,1)\}
$$

they are both the positive $c$-vectors and the non-initial $d$-vectors of $\mathcal{A}_{\mathbf{0}}(B)$.

An immediate and important corollary of Theorem 1.1 is that, for simply laced types, the set $\mathcal{V}(B)$ also coincides with the set of the dimension vectors of all the indecomposable modules of the cluster-tilted algebra $\Lambda(B)$, thereby yielding a representation-theoretic result.

To prove Theorem 1.1 we use the surface realization of cluster algebras [23, 24, 25] for types $A_{n}$ and $D_{n}$. The case $A_{n}$ is easy, but the case $D_{n}$ is (much) more involved. Then we apply the folding method $[21,17]$ to types $D_{n+1}$ and $A_{2 n-1}$ to obtain types $B_{n}$ and $C_{n}$, respectively. Exceptional types are studied by direct inspection with the help of the software by Keller [35] and the cluster algebra package [38] of Sage [50] written by Musiker and Stump; we rely on Corollaries 2.7 and 2.11 to simplify computations in type $E_{8}$. In classical types our derivation is purely combinatorial and does not refer to any results from representation theory. On the one side, this may be unsatisfactory due to the lack of a direct representation-theoretic explanation; on the other side, this is the reason why we get the result easily. In particular, we obtain an alternative proof of the known equality $\mathcal{C}_{+}(B)=\mathcal{D}(B)$ for types $A_{n}$ and $D_{n}$, and also several results on non-simply laced types, for which the representation-theoretic method is not yet fully available.

From the explicit list of positive $c$-vectors and non-initial $d$-vectors provided by Theorem 1.1 we deduce the following result. The statements (1) and (3) generalize to all finite types properties known only for simply-laced types (cf. Corollaries 2.8 and 2.11).

Theorem 1.3. Let B be any skew-symmetrizable matrix of cluster finite type.

1. All c-vectors and d-vectors of $\mathcal{A}_{\mathbf{0}}(B)$ are roots of the root system of $A(B)$. For simply-laced types they are Schur roots.

2. A c-vector (d-vector) of $\mathcal{A}_{\bullet}(B)$ is a real root if and only if its support in $X(B)$ is a tree.

3. The cardinality $\left|\mathcal{C}_{+}(B)\right|=|\mathcal{D}(B)|$ depends only on the cluster type $Z$ of $B$ and it is equal to the number of positive roots in the root system of type $Z$. Explicitly it is equal to $n h / 2$, where $n$ and $h$ are the rank and the Coxeter number of type $Z$ (see Table 1.1).

4. The set $\mathcal{C}_{+}(B)=\mathcal{D}(B)$ only depends on $A(B)$, the Cartan counterpart of $B$.

While proving Theorem 1.1 we also obtain the following interesting result. A skewsymmetrizable integer matrix $B$ is said to be bipartite if the corresponding valued quiver has only sinks and sources; by extension a seed whose $B$-matrix is bipartite is also called bipartite. 
Table 1.1: Coxeter numbers and numbers of positive roots.

\begin{tabular}{|c||c|c|c|c|c|c|c|c|c|}
\hline Type & $A_{n}$ & $B_{n}$ & $C_{n}$ & $D_{n}$ & $E_{6}$ & $E_{7}$ & $E_{8}$ & $F_{4}$ & $G_{2}$ \\
\hline$h$ & $n+1$ & $2 n$ & $2 n$ & $2 n-2$ & 12 & 18 & 30 & 12 & 6 \\
\hline$n h / 2$ & $n(n+1) / 2$ & $n^{2}$ & $n^{2}$ & $n(n-1)$ & 36 & 63 & 120 & 24 & 6 \\
\hline
\end{tabular}

Theorem 1.4. Let $B$ be any skew-symmetrizable matrix of cluster finite type. Any cvector (d-vector) of $\mathcal{A}_{\mathbf{0}}(B)$ occurs in a bipartite seed.

This paper is structured as follows. In Section 2 we give more background and a short survey of the known results on $c$ - and $d$-vectors and their consequences in order to connect our result to representation theory of quivers. In Section 3 we describe the sets $\mathcal{X}(Z)$ and $\mathcal{W}(Z)$ for all the classical finite type $Z$ (i.e. for $A_{n}, B_{n}, C_{n}$ and $D_{n}$ ). We postpone the exceptional types to Appendix B due to their length.

The proofs of Theorems 1.1 and 1.4 for classical types are split into several Propositions and use different techniques. In Section 4 we use the surface realization $([23,24,25])$ of cluster algebras to prove the results for types $A_{n}$ and $D_{n}$. In Section 5 we extend the folding construction of [21] to deal with types $B_{n}$ and $C_{n}$.

The paper is concluded by Section 6 where we prove Theorem 1.3. In Appendix A we add the complete analysis needed in the proof of Propositions 4.10 and 4.11.

\section{More background}

Let us give more background and a short survey of the known results on $c$ - and $d$-vectors and their consequences in order to connect our result to representation theory of quivers. We also propose a conjecture on the root property of $c$-vectors.

\section{$2.1 \quad c$-vectors and $d$-vectors}

We quickly recall the definitions and the basic properties of $c$-vectors and $d$-vectors, which are the main subject of this paper. All the formulas are taken from [28].

Let $\mathbb{Q}(x)$ be the rational function field of algebraically independent variables $x=$ $\left\{x_{i}\right\}_{i \in I}$ over $\mathbb{Q}$, and let $\mathbb{Q}_{+}(x)$ be the subset of $\mathbb{Q}(x)$ which consists of the functions having subtraction-free expressions. The set $\mathbb{Q}_{+}(x)$ is a semifield, and it is called the universal semifield of $x$. We also introduce the tropical semifield $\mathbb{P}_{\text {trop }}(x)$ of $x$ as the multiplicative free abelian group generated by $x$ with the addition $\oplus$ defined by

$$
\prod_{i \in I} x_{i}^{a_{i}} \oplus \prod_{i \in I} x_{i}^{b_{i}}:=\prod_{i \in I} x_{i}^{\min \left(a_{i}, b_{i}\right)} .
$$

Let $\pi_{\text {trop }}: \mathbb{Q}_{+}(x) \rightarrow \mathbb{P}_{\text {trop }}(x)$ be the canonical homomorphism, $x_{i} \mapsto x_{i}, c \mapsto 1\left(c \in \mathbb{Q}_{+}\right)$.

We first describe the $d$-vectors. Since the presence of coefficients is irrelevant, for simplicity, we describe them for a cluster algebra with trivial coefficients. As usual, we start from the initial seed $(B, x)$ with a given skew-symmetrizable integer matrix $B$ and a 
tuple of algebraically independent variables $x=\left\{x_{i}\right\}_{i \in I}$ called the initial cluster variables. We obtain a new seed $\left(B^{\prime}, x^{\prime}\right)$ by the mutation at $k$,

$$
\begin{aligned}
& b_{i j}^{\prime}= \begin{cases}-b_{i j} & i=k \text { or } j=k \\
b_{i j}+b_{i k}\left[b_{k j}\right]_{+}+\left[-b_{i k}\right]_{+} b_{k j} & i, j \neq k,\end{cases} \\
& x_{i}^{\prime}= \begin{cases}x_{k}^{-1}\left(\prod_{j \in I} x_{j}^{\left[b_{j k}\right]_{+}}+\prod_{j \in I} x_{j}^{\left[-b_{j k}\right]_{+}}\right) & i=k \\
x_{i} & i \neq k,\end{cases}
\end{aligned}
$$

where $[a]_{+}=a$ for $a>0$ and 0 otherwise. The elements obtained by sequences of mutations from $x$ are called cluster variables. They are in $\mathbb{Q}_{+}(x)$ since the right hand side of (2.3) is subtraction-free. For any cluster variable $x_{j}^{\prime}$ in some cluster $x^{\prime}=\left\{x_{i}^{\prime}\right\}_{i \in I}$, we define the corresponding $d$-vector $d_{j}^{\prime}=\left(d_{i j}^{\prime}\right)_{i \in I}$ by

$$
\pi_{\text {trop }}\left(x_{j}^{\prime}\right)=\prod_{i \in I} x_{i}^{-d_{i j}^{\prime}} .
$$

The matrix $D^{\prime}=\left(d_{i j}^{\prime}\right)_{i, j \in I}$ is called the $D$-matrix of $x^{\prime}$. This definition of the $d$-vector $d_{j}^{\prime}$ agrees with an alternative and more familiar definition as the tuple of the exponents of the "denominator" of the Laurent polynomial expression of $x_{j}^{\prime}$,

$$
x_{j}^{\prime}=\frac{P(x)}{\prod_{i \in I} x_{i}^{d_{i j}^{\prime}}},
$$

where $P(x)$ is a polynomial in $x=\left\{x_{i}\right\}_{i \in I}$ not divisible by any $x_{i}$. (Note that the celebrated Laurent phenomenon [26] does not necessarily imply that the components of the $d$-vector for a non-initial cluster variable are all nonnegative.) For cluster variables $x_{j}^{\prime \prime}$ and $x_{j}^{\prime}$ which are connected by a mutation $\left(B^{\prime \prime}, x^{\prime \prime}\right)=\mu_{k}\left(B^{\prime}, x^{\prime}\right)$, we have a recursion relation for the corresponding $d$-vectors, which is the tropicalization of (2.3),

$$
d_{i j}^{\prime \prime}= \begin{cases}-d_{i k}^{\prime}+\max \left(\sum_{\ell \in I} d_{i \ell}^{\prime}\left[b_{\ell k}^{\prime}\right]_{+}, \sum_{\ell \in I} d_{i \ell}^{\prime}\left[-b_{\ell k}^{\prime}\right]_{+}\right) & j=k \\ d_{i j}^{\prime} & j \neq k .\end{cases}
$$

Next we describe the $c$-vectors. We need another tuple of algebraically independent variables $y=\left(y_{i}\right)_{i \in I}$ called the initial coefficients. They mutate, along with the mutation of the exchange matrix $B$, with the exchange relation at $k$ given by

$$
y_{i}^{\prime}= \begin{cases}y_{i}^{-1} & i=k \\ y_{i} \frac{\left(1+y_{k}\right)^{\left[-b_{k i}\right]_{+}}}{\left(1+y_{k}^{-1}\right)^{\left[b_{k i}\right]_{+}}} & i \neq k .\end{cases}
$$


The elements of $\mathbb{Q}_{+}(y)$ obtained by successive mutations are called coefficients. For any coefficient $y_{j}^{\prime}$ in a coefficient tuple $y^{\prime}=\left(y_{i}^{\prime}\right)_{i \in I}$, we define the corresponding $c$-vector $c_{j}^{\prime}=\left(c_{i j}^{\prime}\right)_{i \in I}$ by

$$
\pi_{\text {trop }}\left(y_{j}^{\prime}\right)=\prod_{i \in I} y_{i}^{c_{i j}^{\prime}}
$$

The matrix $C^{\prime}=\left(c_{i j}^{\prime}\right)_{i, j \in I}$ is called the $C$-matrix of $y^{\prime}$.

For coefficients $y_{j}^{\prime \prime}$ and $y_{j}^{\prime}$ which are connected by a mutation $\left(B^{\prime \prime}, y^{\prime \prime}\right)=\mu_{k}\left(B^{\prime}, y^{\prime}\right)$, we have a recursion relation for the corresponding $c$-vectors, which is the tropicalization of $(2.7)$,

$$
c_{i j}^{\prime \prime}= \begin{cases}-c_{i j}^{\prime} & j=k \\ c_{i j}^{\prime}+c_{i k}^{\prime}\left[b_{k j}^{\prime}\right]_{+}+\left[-c_{i k}^{\prime}\right]_{+} b_{k j}^{\prime} & j \neq k .\end{cases}
$$

This definition of $c$-vectors agrees with an alternative and more familiar definition as column vectors of the bottom half square matrix of the extended exchange matrices of $\mathcal{A}_{\bullet}(B)$ (cf. (2.2)).

\subsection{Sign-coherence Conjecture}

Fomin and Zelevinsky made the following fundamental conjecture on $c$ - and $d$-vectors, which plays an important role in the structure theory of cluster algebras (e.g., [28, 43]).

Conjecture 2.1 (Sign-coherence Conjecture). Let $B$ be any skew-symmetrizable matrix.

(i) $\left[28\right.$, Conjecture $5.5 \&$ Proposition 5.6] Any $c$-vector of $\mathcal{A}_{\bullet}(B)$ is a nonzero vector, and its components are either all non-negative or all non-positive.

(ii) $[28$, Conjectures $7.4 \& 7.5]$ Any non-initial $d$-vector of $\mathcal{A}_{\bullet}(B)$ is a nonzero vector, and its components are all nonnegative.

The first part of the conjecture is equivalent to the fact that the constant term of any $F$-polynomial of $\mathcal{A}_{\mathbf{0}}(B)$ is one [28], which is proved for any skew-symmetric matrix $B$ $[19,39,45]$, and also for a large class of skew-symmetrizable matrices [16], in particular, for any skew-symmetrizable matrix which is mutation equivalent to an acyclic one.

The second part of the conjecture is proved, for example, for any skew-symmetric matrix $B$ arising from a surface [24], and more cases follow from the results in the rest of this section.

\subsection{Root Conjecture}

Recall that a skew-symmetric matrix $B=\left(b_{i j}\right)_{i, j \in I}$ can be identified with a quiver $Q(B)$ without loops and 2-cycles by attaching $b_{i j}$ arrows from vertex $i$ to vertex $j$ if $b_{i j}>0$. This correspondence can be extended to the one between skew-symmetrizable matrices and valued quivers (see [20]). 
Let $\Delta(A)$ be the root system associated with a symmetrizable Cartan matrix $A$, and let $\left\{\alpha_{i}\right\}_{i \in I}$ be its simple roots [34]. A root $\alpha=\sum_{i \in I} c_{i} \alpha_{i}$ of $\Delta(A)$ is naturally identified with, either all nonnegative or all non-positive, nonzero integer vector $\left(c_{i}\right)_{i \in I}$. It is said to be real if there is an element $w$ of the Weyl group of $\Delta(A)$ such that $w(\alpha)$ is a simple root; otherwise it is said to be imaginary. It is known that a root $\alpha$ is real if and only if $(\alpha, \alpha)_{T A}={ }^{t} \alpha T A \alpha>0$, where $T$ is any diagonal matrix with positive diagonal entries such that $T A$ is symmetric. See [34] for details.

In the study of cluster algebras, it becomes more and more apparent that there is some intimate interplay among three kinds of algebras, namely, cluster algebras, path algebras, and (quantized) Kac-Moody algebras. Naturally, root systems provide the common underlying structure. The starting point of the interplay is Kac's theorem, which generalizes celebrated Gabriel's theorem. Let $k$ be an algebraically closed field below.

Theorem 2.2 (Kac's Theorem [32,33]). Let B be any skew-symmetric matrix. Then, there exists an indecomposable module of the path algebra $k Q(B)$ with dimension vector $\alpha$ if and only if $\alpha$ is a positive root of $\Delta(A(B))$.

In the above correspondence, if a positive root is the dimension vector of some indecomposable $k Q(B)$-module $M$ such that $\operatorname{End}_{k Q(B)}(M)=k$, then it is called a Schur root. We use this notion later.

In view of cluster algebras, the extension of Theorem 2.2 to the valued quivers is desired and expected. Unfortunately, it is not fully achieved yet [31, 18]. Nevertheless, the perspective presented above guides us to the following natural refinement of Conjecture 2.1, jointly proposed with Andrei Zelevinsky.

Conjecture 2.3 (Root Conjecture). For any skew-symmetrizable matrix $B$ any $c$-vector of $\mathcal{A}_{\bullet}(B)$ is a root of $\Delta(A(B))$.

As for $d$-vectors, they also satisfy the same root property in many known cases. However Marsh and Reiten recently found, in cluster affine type $A$, an example of a $d$-vector which is not a root of $\Delta(A(B))$ [36]. We thank Robert Marsh and Idun Reiten for sharing with us this counterexample.

\subsection{Results for finite type}

Cluster algebras of finite type were studied in detail by various authors. Here we collect some of the known properties of their $c$ - and $d$-vectors along with some consequences which are relevant to the present paper. For simplicity, we assume that a skew-symmetrizable matrix $B$ is indecomposable in this section.

The connection between the $d$-vectors and the root systems of finite type was first discovered by Fomin and Zelevinsky [27]. Recall that a skew-symmetrizable integer matrix $B$ is said to be bipartite if the corresponding valued quiver has only sinks and sources.

Theorem 2.4 ([27, Theorem 1.9]). For any skew-symmetrizable bipartite matrix $B$ whose Cartan counterpart $A(B)$ is of finite type, the set $\mathcal{D}(B)$ coincides with the set of all the positive roots of $\Delta(A(B))$. 
The requirement of $B$ being bipartite was lifted later on in [55]. In particular, in the skew-symmetric case, combining the above result with Gabriel's theorem, we get that the set $\mathcal{D}(B)$ also coincides with the set of all the dimension vectors of the path algebra $k Q(B)$. This result triggered the intensive representation-theoretic study of cluster algebras in the past decade.

For a skew-symmetric matrix $B$ of cluster finite type, let $\Lambda(B)$ be the corresponding cluster-tilted algebra, which is the path algebra of the quiver $Q(B)$ modulo the relations described by [6, Theorem 4.2]. Note that any indecomposable $\Lambda(B)$-module can also be regarded as an indecomposable $k Q(B)$-module. Let $\operatorname{Dim}(\Lambda(B))$ be the set of the dimension vectors of all the indecomposable $\Lambda(B)$-modules.

The following theorem by Caldero, Chapoton, and Schiffler [13], and by Buan, Marsh, and Reiten [7], extended Theorem 2.4 to any skew-symmetric matrix $B$ of cluster finite type.

Theorem 2.5 ([13, Theorem 4.4 \& Remark 4.5], [7, Theorem 2.2]). For any skewsymmetric matrix $B$ of cluster finite type, the sets $\mathcal{D}(B)$ and $\operatorname{Dim}(\Lambda(B)$ ) coincide.

On the other hand, Nájera Chávez recently proved a parallel theorem for $c$-vectors.

Theorem 2.6 ([42, Theorem 4],[40]). For any skew-symmetric matrix $B$ of cluster finite type, the sets $\mathcal{C}_{+}(B)$ and $\operatorname{Dim}(\Lambda(B))$ coincide.

The inclusion $\mathcal{C}_{+}(B) \subset \operatorname{Dim}(\Lambda(B))$ is a special case of [42, Theorem 4] (see Theorem 2.16), while the opposite inclusion is due to a yet unpublished result communicated to us by Alfredo Nájera Chávez [40].

We have the following immediate corollary of Theorems 2.5 and 2.6.

Corollary 2.7. For any skew-symmetric matrix $B$ of cluster finite type, the sets $\mathcal{C}_{+}(B)$ and $\mathcal{D}(B)$ coincide.

It is known that, for any indecomposable $\Lambda(B)$-module $M, \operatorname{End}_{\Lambda(B)}(M)=k$ holds (and therefore $\operatorname{End}_{k Q(B)}(M)=k$ ) [4, Section 8]. Thus, we have another corollary of Theorems 2.5 and 2.6.

Corollary 2.8. For any skew-symmetric matrix $B$ of cluster finite type, all positive cvectors and all non-initial d-vectors are Schur roots of $\Delta(A(B))$.

For any skew-symmetric matrix $B$ of cluster finite type, let us introduce the set

$$
\operatorname{Ind}(\Lambda(B))=\{\text { all indecomposable } \Lambda(B) \text {-modules }\} \text {. }
$$

The following remarkable fact holds.

Theorem 2.9 ([7, Corollary 2.4]). For any skew-symmetric matrix $B$ of cluster finite type, the cardinality $|\operatorname{Ind}(\Lambda(B))|$ only depends on the cluster type $Z$ of $B$; it is equal to the number of positive roots of the root system of type $Z$. 
The dimension map

$$
\underline{\operatorname{dim}}: \operatorname{Ind}(\Lambda(B)) \rightarrow \operatorname{Dim}(\Lambda(B))
$$

is surjective by definition. Actually, it is bijective by the following theorem.

Theorem 2.10. [47, Theorem 1] For any skew-symmetric matrix B of cluster finite type, the map dim in (2.11) is injective.

We have an immediate corollary of Theorems 2.5, 2.6, 2.9, and 2.10.

Corollary 2.11. For any skew-symmetric matrix $B$ of cluster finite type, the cardinality $\left|\mathcal{C}_{+}(B)\right|=|\mathcal{D}(B)|$ only depends on the cluster type $Z$ of $B$, and it is equal to the number of positive roots of the root system of type $Z$.

\subsection{More general results}

For completeness, we summarize some general results on $c$ - and $d$-vectors beyond finite type and also give some examples, though we do not use them in the rest of the paper.

A skew-symmetrizable matrix $B$ is acyclic if the corresponding valued quiver $Q(B)$ is acyclic, i.e., without oriented cycles. Let us first discuss the case of an acyclic skewsymmetric matrix $B$. Under this hypothesis, the cluster tilted algebra $\Lambda(B)$ is the path algebra $k Q(B)$ itself because there is no relation to be imposed. A $k Q(B)$-module $M$ is said to be rigid if $\operatorname{Ext}_{k Q(B)}^{1}(M, M)=0$.

The following two theorems completely describe the $c$ - and $d$-vectors in this case:

Theorem 2.12 ([14, Theorem 4], [10, Theorem 2.3]). For any acyclic skew-symmetric matrix $B$, the set $\mathcal{D}(B)$ coincides with the set of the dimension vectors of all the rigid indecomposable $k Q(B)$-modules.

Theorem 2.13 ([42, Theorem 1]). For any acyclic skew-symmetric matrix $B$, the set $\mathcal{C}_{+}(B)$ coincides with the set of the dimension vectors of all the rigid indecomposable $k Q(B)$-modules.

Recall that, when $Q(B)$ is acyclic, the following formula holds [1]:

$$
\frac{1}{2}(\underline{\operatorname{dim}} M, \underline{\operatorname{dim}} M)_{A(B)}=\operatorname{dim} \operatorname{End}_{k Q(B)}(M)-\operatorname{dim}_{\operatorname{Ext}_{k Q(B)}^{1}}(M, M)
$$

It follows that $\alpha$ is the dimension vector of a rigid indecomposable $k Q(B)$-module if and only if it is a real Schur root. Therefore, we have an alternative form of Theorems 2.12 and 2.13 .

Corollary 2.14 ([42, Theorem 1]). For any acyclic skew-symmetric matrix $B$, both the sets $\mathcal{D}(B)$ and $\mathcal{C}_{+}(B)$ coincide with the set of all the real Schur roots of $\Delta(A(B))$.

Both Theorem 2.13 and Corollary 2.14 are partially extended to the acyclic skewsymmetrizable matrices. (The sign-coherence of $c$-vectors is covered by [16].) 
Theorem 2.15 ([46, Theorem 1.1], [49, Theorem 1]). For any acyclic skew-symmetrizable matrix $B$, any positive c-vector is a real positive root of $\Delta(A(B))$; moreover, it is the dimension vector of a rigid indecomposable representation of the valued quiver $Q(B)$.

Finally, beyond finite type and the acyclic case, the following result is so far the most general result on $c$-vectors; in particular, it ensures and strengthens Conjecture 2.3 for any skew-symmetric matrix $B$.

Theorem 2.16 ([42, Theorem 4]). For any skew-symmetric matrix B, any positive cvector of $\mathcal{A}_{\mathbf{0}}(B)$ is the dimension vector of some rigid indecomposable module $M$ of the Jacobian algebra $J(Q(B), W)$ of the quiver $Q(B)$ with generic potential $W$ such that $\operatorname{End}_{J(Q(B), W)}(M)=k$. In particular, any positive c-vector of $\mathcal{A}_{\bullet}(B)$ is a Schur root of $\Delta(A(B))$.

On the other hand the behavior of the $d$-vectors is rather complicated as studied in $[9,5,36]$. What was observed therein is a deficiency phenomenon: in some situations the $d$-vector of a cluster variable $x_{i}^{\prime}$ is smaller than the dimension vector of the rigid indecomposable $\Lambda(B)$-module associated with $x_{i}^{\prime}$.

We conclude this short survey by presenting two illuminating examples beyond finite type and the acyclic case.

Example 2.17. Type $A_{2}^{(1)}$. Consider the skew-symmetric matrix $B$ corresponding the following non-acyclic quiver:

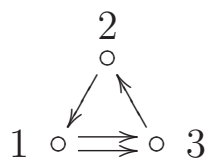

It is mutation equivalent to the following acyclic quiver whose Cartan counterpart is the Cartan matrix of affine type $A_{2}^{(1)}$.

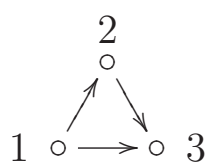

This cluster algebra $\mathcal{A}_{\bullet}(B)$ is studied in detail by [15]. In particular, the non-initial $d$-vectors of $\mathcal{A}_{\bullet}(B)$ are given by [15, Lemma 3.3]:

$$
(0,1,0),(1,1,1),(a, 0, a+1),(a+1,0, a),(a, 1, a+1),(a+1,1, a), \quad a \geqslant 0 .
$$

Moreover, it is not difficult to show that the positive $c$-vectors of $\mathcal{A}_{\bullet}(B)$ are also given by the same list. Therefore, in this case $\mathcal{C}_{+}(B)=\mathcal{D}(B)$ holds, even though $B$ is not acyclic. Thus, any non-initial $d$-vector is a Schur root of $\Delta(A(B))$ by Theorem 2.16. Note that the $d$-vector $(1,1,1)$ in $(2.13)$ is the simplest example which shows the deficiency phenomenon [9, Example 7.2], where the dimension vector of the corresponding representation is $(1,2,1)$. Nevertheless, the $d$-vector $(1,1,1)$ is still a Schur root. We also note that among the vectors in (2.13), the last two are imaginary roots for $a \geqslant 1$. 
Example 2.18. Markov quiver. We consider the skew-symmetric matrix $B$ such that the corresponding quiver is the following non-acyclic one.

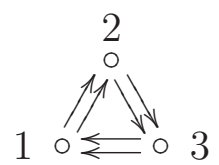

This is known as the Markov quiver, and the positive $c$-vectors of $\mathcal{A}_{\bullet}(B)$ are given by the permutations of the following vectors [41, Theorem 3.1.2]:

$$
(1,2,2),(a+1, b+1, a+b+1),(a-1, b-1, a+b-1),
$$

where $1 \leqslant a \leqslant b$, and $a$ and $b$ are coprime. The cluster algebra $\mathcal{A}_{\bullet}(B)$ has a surface realization by a once-punctured torus. Using the same technique as in Section 4, it can be shown that the non-initial $d$-vectors are given by the permutations of the vectors in (2.14) of the form

$$
(a-1, b-1, a+b-1) .
$$

So this gives the first example in which the sets $\mathcal{D}(B)$ and $\mathcal{C}_{+}(B)$ do not coincide. Nevertheless, $\mathcal{D}(B) \subset \mathcal{C}_{+}(B)$ so any non-initial $d$-vector is still a Schur root of $\Delta(A(B))$ by Theorem 2.16.

The above examples may suggest that the property $\mathcal{D}(B) \subset \mathcal{C}_{+}(B)$ holds in general but this is not true due to the counterexample of [36].

\section{$3 \quad$ The sets $\mathcal{X}(Z)$ and $\mathcal{W}(Z)$ for classical types}

Let $Z$ be any type in one of the four infinite families (i.e. $Z$ is one of $A_{n}, B_{n}, C_{n}$, or $D_{n}$ for some positive integer $n$ ). In this section we provide a description of all the diagrams in $\mathcal{X}(Z)$ and define the list $\mathcal{W}(Z)$ of allowed weighted diagram for each type required by Theorem 1.1. The analogous sets for the remaining finite types will be presented in Appendix B.

\subsection{Type $A_{n}$}

The following is a direct consequence of Proposition 2.4 in [11].

Proposition 3.1. A diagram $X$ is in $\mathcal{X}\left(A_{n}\right)$ if and only if the following conditions are satisfied:

- $X$ has $n$ vertices, is simply laced and connected;

- every cycle in $X$ is a triangle;

- each vertex in $X$ has at most four neighbours; 
- if a vertex has three neighbours then exactly two of them are adjacent;

- if a vertex has four neighbours then they can be partitioned into two disjoint sets, containing two elements each, and such that the two neighbouring vertices $i$ and $j$ are adjacent if and only if $\{i, j\}$ is one of those sets.

An example of Dynkin diagram in $\mathcal{X}\left(A_{n}\right)$ is presented in Figure 3.1 to illustrate its "quasi-tree" nature.

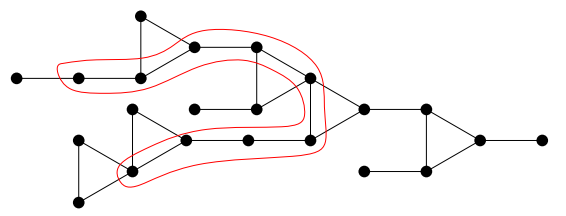

Figure 3.1: A typical element $X$ in $\mathcal{X}\left(A_{22}\right)$. The highlighted part is an element of $\mathcal{W}\left(A_{22}\right)$ embedded in $X$.

The set $\mathcal{W}\left(A_{n}\right)$ consists of type $A$ Dynkin diagrams (strings) with at most $n$ vertices. All the multiplicities are 1 . Elements of $\mathcal{W}\left(A_{n}\right)$ are pictorially presented as follows.

An example of an embedding of such a string in a diagram of $\mathcal{X}\left(A_{n}\right)$ is highlighted in Figure 3.1. Note that, as explained in the introduction, an embedding of an element of $\mathcal{W}\left(A_{n}\right)$ in a diagram $X$ is given by a full sub-diagram; therefore at most two vertices of each triangle of $X$ can belong to it. It follows that an embedding of a string is uniquely determined by the positions of its endpoints [44]. Note that the equality $\mathcal{D}(B)=\mathcal{V}(B)$ is known in this case by $[12,44,53]$.

The building block of Dynkin diagrams for classical types is given by diagrams of type $A_{n}$. While stating the analogous results for other types we will use the convention $\mathcal{X}\left(A_{0}\right)=\emptyset$.

\subsection{Type $B_{n}$}

As usual for Dynkin diagrams we put $a_{i j} a_{j i}$ edges between $i$ and $j$ and the inequality sign on the edges refers to the relation among the lengths of the corresponding simple roots. This convention agrees with [34] and it is the opposite to the convention used in [3]. To make it more explicit the Cartan matrix

$$
\left(\begin{array}{cc}
2 & -1 \\
-2 & 2
\end{array}\right)
$$

corresponds in this paper to the following Dynkin diagram (labels correspond to the rows of $B)$. 


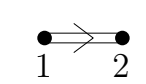

Proposition 3.2. A diagram with $n$ vertices $(n \geqslant 2)$ is in $\mathcal{X}\left(B_{n}\right)$ if and only if it is one of the two in Figure 3.2 where $X^{(i)}$ is any diagram in $\mathcal{X}\left(A_{m}\right)$ for a suitable $m \geqslant 0$.

We postpone the proof to Section 5.2.

The weighted diagrams in $\mathcal{W}\left(B_{n}\right)$ are those in Figure 3.3. As we will see they are obtained from (some of) those in $\mathcal{W}\left(D_{n+1}\right)$ by folding.

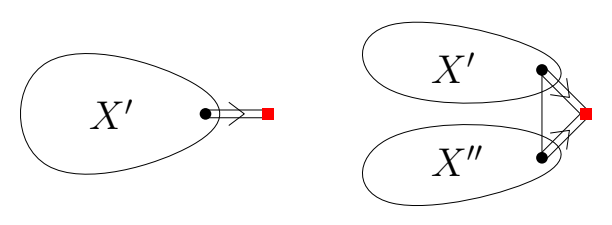

Figure 3.2: Elements of $\mathcal{X}\left(B_{n}\right)$ for $n \geqslant 2 ; X^{(i)}$ is any diagram in $\mathcal{X}\left(A_{m}\right)$ for a suitable $m \geqslant 0$. The nodes marked as red squares are the images of those permuted by $\sigma$ in Proposition 5.4.
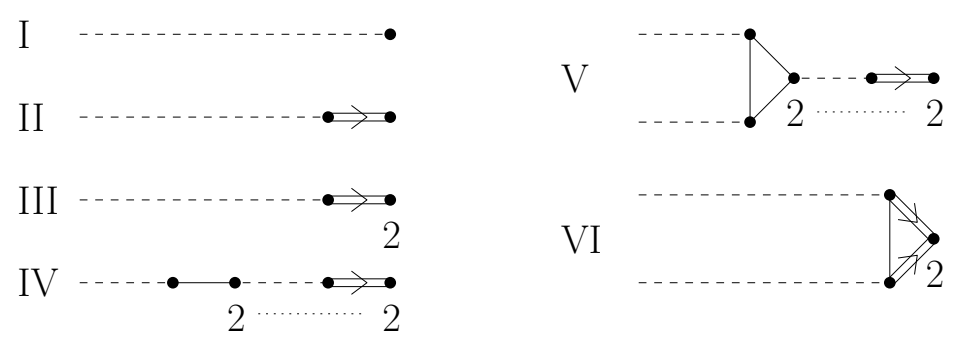

Figure 3.3: The set $\mathcal{W}\left(B_{n}\right)$. Dotted lines are strings of any length; multiplicity of all the nodes of each such string are the same as their ending points. Solid lines can't be omitted. We will use the above drawing conventions thorough the rest of the paper.

\subsection{Type $C_{n}$}

Proposition 3.3. A diagram with $n$ vertices $(n \geqslant 2)$ is in $\mathcal{X}\left(C_{n}\right)$ if and only if it is one of the two in Figure 3.4 where $X^{(i)}$ is any diagram in $\mathcal{X}\left(A_{m}\right)$ for a suitable $m \geqslant 0$.

We postpone the proof to Section 5.2.

Remark 3.4. The results of Propositions 3.2 and 3.3 were claimed in [38] and encoded in the cluster algebra package of Sage. The details will appear in [51]. The same result also appeared in [30].

The weighted diagrams in $\mathcal{W}\left(C_{n}\right)$ are those in Figure 3.5; as we will see they are all the weighted diagrams that can be obtained by folding a string embedded on a diagram in $\mathcal{X}\left(A_{2 n-1}\right)$. 

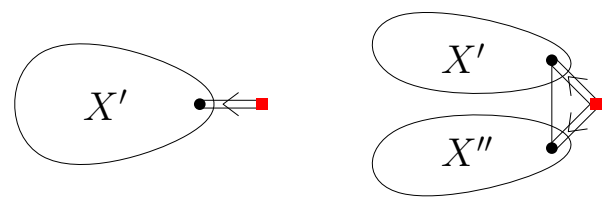

Figure 3.4: Elements of $\mathcal{X}\left(C_{n}\right)$ for $n \geqslant 2 ; X^{(i)}$ is any diagram in $\mathcal{X}\left(A_{m}\right)$ for a suitable $m \geqslant 0$. The nodes marked as red squares are the images of the fixed point under the action of $\sigma$ in Proposition 5.4.
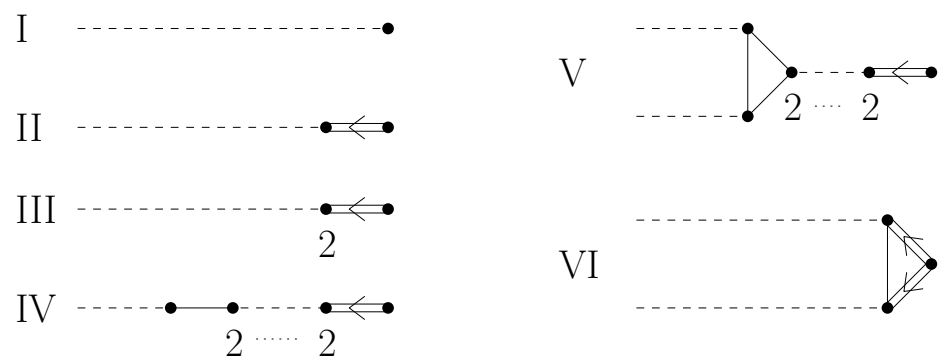

Figure 3.5: The set $\mathcal{W}\left(C_{n}\right)$. We use the same drawing conventions of Figure 3.3.

\subsection{Type $D_{n}$}

From Theorem 3.1 in [54] together with Proposition 3.1 we get the following description of $\mathcal{X}\left(D_{n}\right)$. Note that the same result can also be obtained easily from the surface realization we use in Section 4.

Proposition 3.5. A diagram with $n$ vertices $(n \geqslant 4)$ is in $\mathcal{X}\left(D_{n}\right)$ if and only if it is one of the four in Figure 3.6 where $X^{(i)}$ is any diagram in $\mathcal{X}\left(A_{m}\right)$ for a suitable $m \geqslant 0$.

(a)

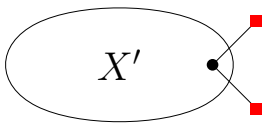

(b)

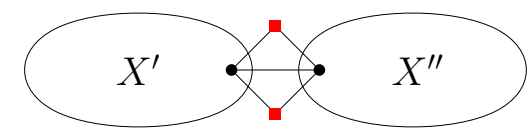

(c)

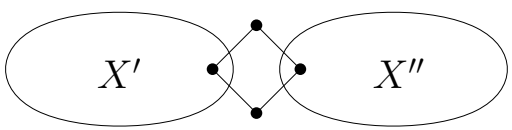

(d)

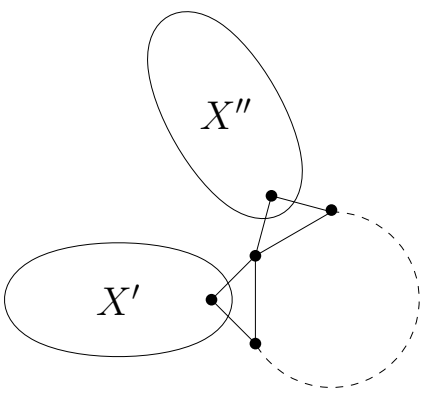

Figure 3.6: Elements of $\mathcal{X}\left(D_{n}\right)$ for $n \geqslant 4 ; X^{(i)}$ is any diagram in $\mathcal{X}\left(A_{m}\right)$ for a suitable $m \geqslant 0$. The nodes marked as red squares are the one permuted by $\sigma$ in Remark 5.4. Case (d) consists of a central cycle with, possibly, type- $A$ components attached to its sides.

The set $\mathcal{W}\left(D_{n}\right)$ consists of all the weighted diagrams in Figure 3.7. 


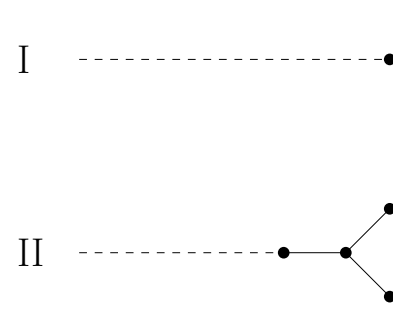

V
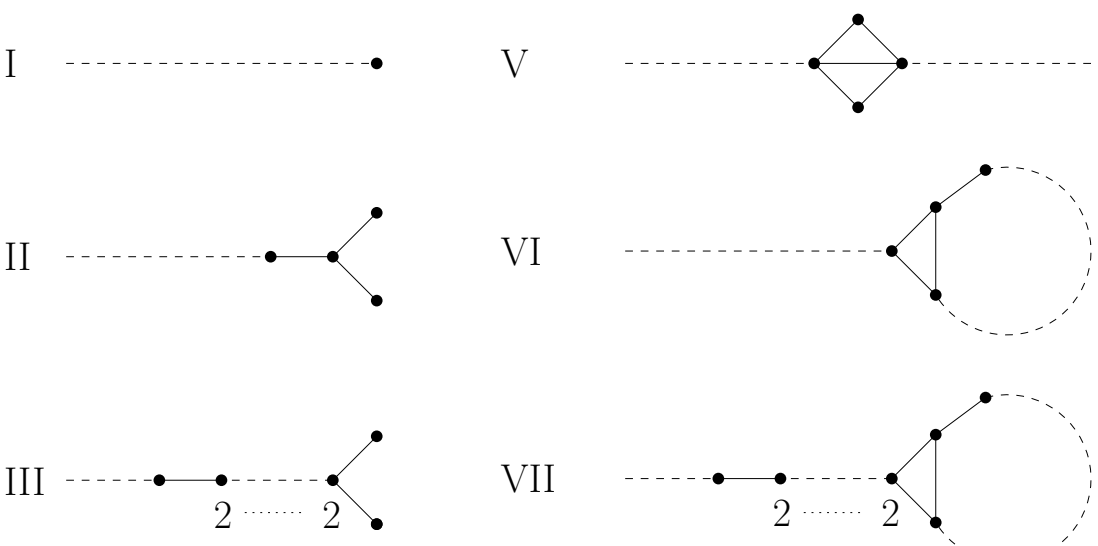

VII
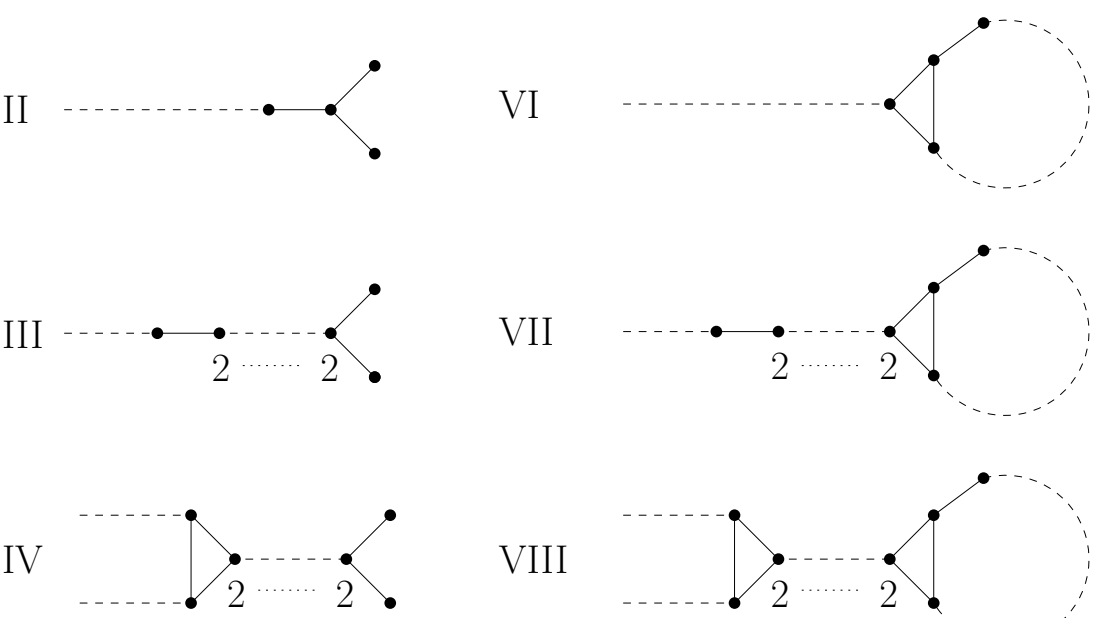

VIII

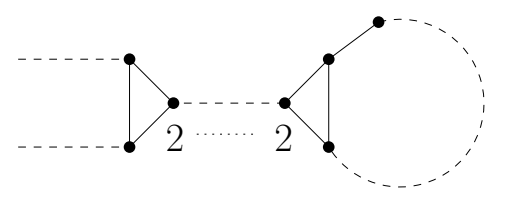

Figure 3.7: The set $\mathcal{W}\left(D_{n}\right)$. We use the same drawing conventions of Figure 3.3.

\subsection{Examples}

To illustrate how to read the data presented in this section let us consider two examples.

Example 3.6. Let $B$ be the matrix

$$
\left(\begin{array}{ccccc}
0 & 1 & 0 & 0 & 0 \\
-1 & 0 & 1 & -1 & 0 \\
0 & -1 & 0 & 1 & -1 \\
0 & 1 & -1 & 0 & 1 \\
0 & 0 & 1 & -1 & 0
\end{array}\right)
$$

of cluster type $D_{5}$. The diagram $X(B)$ and the set of positive $c$-vectors (and non-initial $d$-vectors) of $\mathcal{A}_{\mathbf{e}}(B)$ are shown in Figures 3.8 and 3.9 respectively. Note that any skewsymmetric matrix of cluster type $D_{5}$ whose entries are the same as the entries of $B$ in absolute value produces the same $X(B)$ and $\mathcal{V}(B)$.

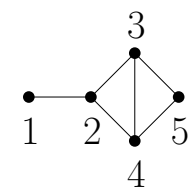

Figure 3.8: $X(B)$ for Example 3.6. Labels refer to the rows of $B$. 


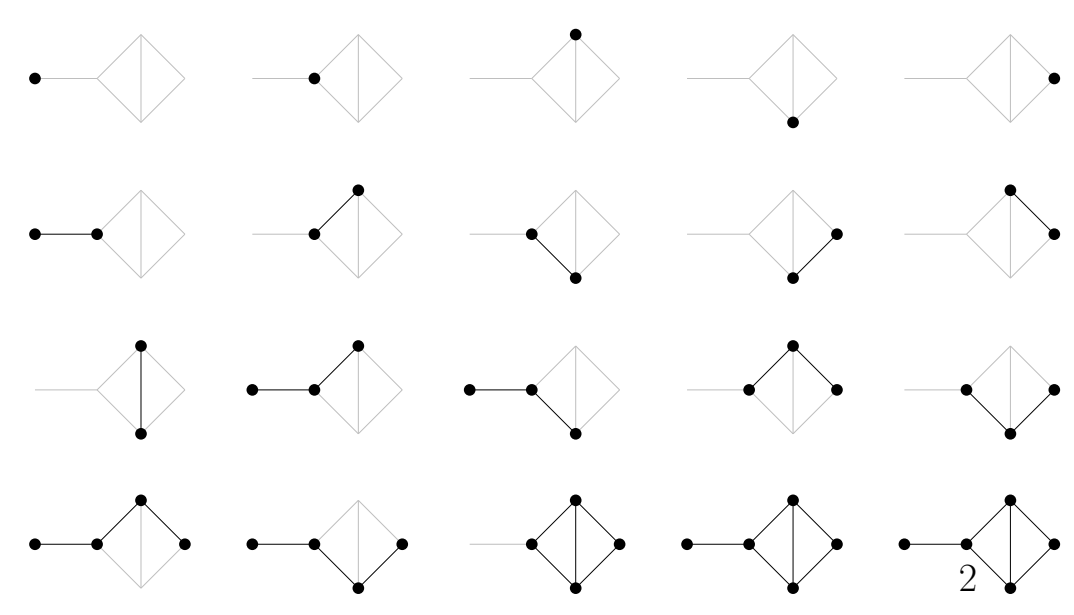

Figure 3.9: $\mathcal{V}(B)$ for Example 3.6.

Example 3.7. Let $B$ be the matrix

$$
\left(\begin{array}{rrrrr}
2 & -1 & 0 & 0 & 0 \\
-1 & 2 & -1 & 0 & 0 \\
0 & -1 & 2 & -2 & -1 \\
0 & 0 & -1 & 2 & -1 \\
0 & 0 & -1 & -2 & 2
\end{array}\right)
$$

of cluster type $C_{5}$. The diagram $X(B)$ and the set of positive $c$-vectors (and non-initial $d$-vectors) of $\mathcal{A}_{\bullet}(B)$ are shown in Figures 3.10 and 3.11 respectively.

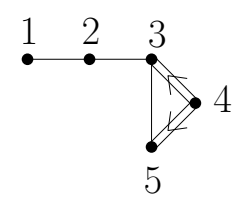

Figure 3.10: $X(B)$ for Example 3.7. Labels refer to the rows of $B$.

\section{Types $A_{n}$ and $D_{n}$ : the surface method}

In this section we prove Theorem 1.1 for types $A_{n}$ and $D_{n}$.

\subsection{The surface method for types $A_{n}$ and $D_{n}$.}

To describe $c$-vectors and $d$-vectors in types $A_{n}$ and $D_{n}$ we do not need to use the construction of [24] in its full generality so we can slightly simplify the definitions; the reader interested in the general theory can find a comprehensive review in [37]. 


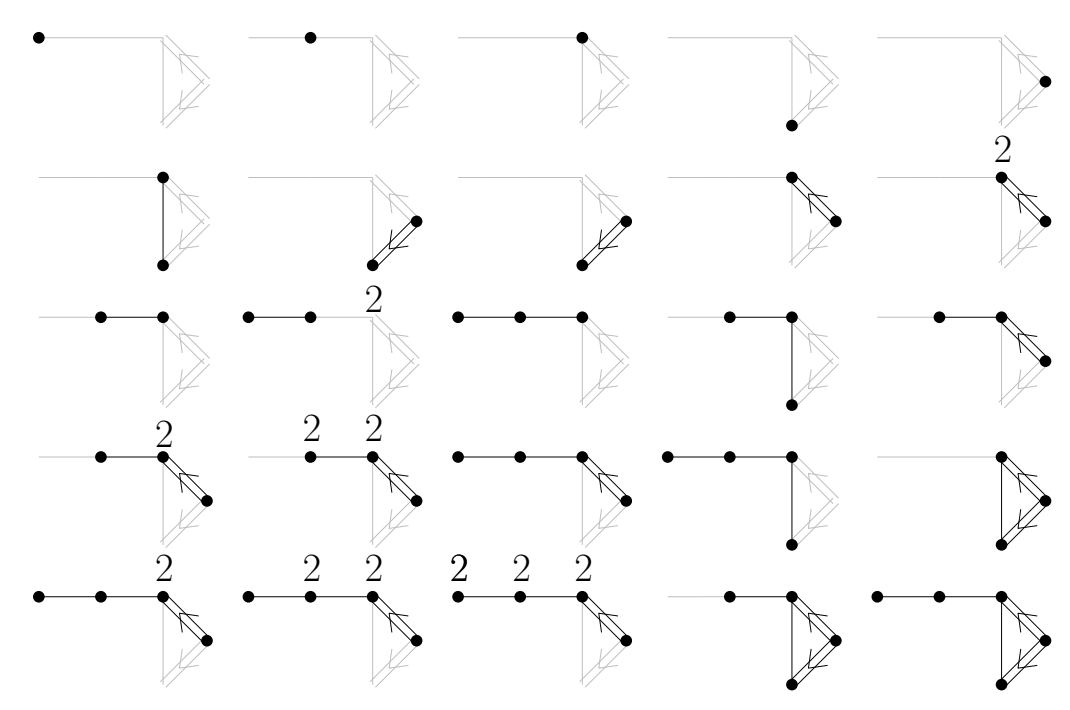

Figure 3.11: $\mathcal{V}(B)$ for Example 3.7.

Unless otherwise specified, by surface $S$ we mean one of the following:

- (type $\left.A_{n}\right)$ a disk with $n+3$ marked points on its boundary $(n \geqslant 1)$;

- (type $\left.D_{n}\right)$ a disk with $n$ marked points on the boundary $(n \geqslant 4)$ and one, the puncture, in its interior.

We denote the set of marked points by $M$.

Definition 4.1. A (tagged) arc is an homotopy class of curves $\gamma$ in the interior of $S \backslash M$ having no self intersections, connecting two distinct points of $M$, and not cutting out (together with a boundary component of $S$ ) an unpunctured bigon. Due to the limitations imposed on the kinds of surfaces we consider there are only two possible types of arcs: chords, connecting two marked point on the boundary of $S$, and radii, connecting a point on the boundary with the puncture. Radii comes in two flavours: plain and notched; to distinguish them in figures we will put a cross on notched arcs.

Remark 4.2. Note that this is not the usual definition of tagged arcs, in particular for general surfaces there is a tagging attached to each endpoint of any $\gamma$. Another difference from the general case is that we are not allowing loops (arcs with coinciding endpoints).

We need not consider ideal arcs as defined by [24] so we can drop the adjective "tagged" without generating confusion. To any pair of arcs $\gamma$ and $\delta$ we can associate an integer as follows.

Definition 4.3 ([24, Definition 8.4]). The intersection pairing of $\gamma$ and $\delta$ is the integer $(\gamma \mid \delta)$ defined according to these rules:

1. if $\gamma$ and $\delta$ coincide then $(\gamma \mid \delta)=-1$; 
2. if $\gamma$ and $\delta$ are homotopic radii with different tagging then $(\gamma \mid \delta)=0$;

3. if $\gamma$ and $\delta$ are non-homotopic radii then $(\gamma \mid \delta)=0$ if they are tagged in the same way and $(\gamma \mid \delta)=1$ if their tagging is different;

4. in any other case, set $(\gamma \mid \delta)$ to be the minimal number of intersections between $\gamma$ and $\delta$.

Two arcs $\gamma$ and $\delta$ are said to be compatible if their intersection pairing is non-positive. A triangulation $\Gamma$ of $S$ is a maximal (by inclusion) set of pairwise compatible arcs.

Remark 4.4. Definition 4.3 is symmetric; this is not the case for a general surface where loops are allowed (see [24, Example 8.5]).

In view of [24, Theorem 7.9] each triangulation of $S$ has $n$ arcs in it and given a triangulation $\Gamma$ and one of its arcs $\gamma$, there is a unique other arc $\gamma^{\prime}$ such that

$$
\Gamma^{\prime}=(\Gamma \backslash\{\gamma\}) \cup\left\{\gamma^{\prime}\right\}
$$

is again a triangulation of $S$. The operation of replacing $\gamma$ with $\gamma^{\prime}$ is called a flip.

To any triangulation $\Gamma$ associate a skew-symmetric matrix $B(\Gamma)=\left(b_{\gamma \delta}^{\Gamma}\right)_{\gamma, \delta \in \Gamma}$ setting

$$
b_{\gamma \delta}^{\Gamma}:= \begin{cases}1 & \text { if } \gamma \text { rotates counterclockwise to } \delta \\ -1 & \text { if } \gamma \text { rotates clockwise to } \delta \\ 0 & \text { if both or none of the previous conditions hold }\end{cases}
$$

where $\gamma$ is said to rotate counterclockwise (resp. clockwise) to $\delta$ if they are not homotopic, they share an endpoint and, in a neighbourhood of this point, $\gamma$ can be deformed counterclockwise (resp. clockwise), without crossing any other arc of $\Gamma$, to coincide with $\delta$.

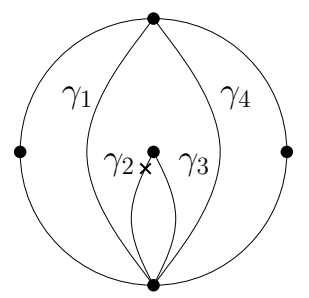

Figure 4.1: In this triangulation $b_{21}=b_{31}=b_{14}=b_{42}=b_{43}=1$ while $b_{23}=0$.

By [24, Theorem 7.11] the above assignment produces a bijection between triangulations of a type $A_{n}$ (resp. $D_{n}$ ) surface and unlabeled seeds of the coefficient-free cluster algebra of the same type. In particular cluster variables are in bijection with arcs and if two seeds are obtained from one another exchanging the cluster variables $x_{\gamma}$ and $x_{\gamma^{\prime}}$ then the corresponding triangulations are related by the flip of $\gamma$ into $\gamma^{\prime}$.

To keep track of principal coefficients we use laminations as explained in [25]. For each marked point $p$ on the boundary of $S$ fix a neighbouring point $p^{\prime}$ obtained sliding $p$ clockwise on the boundary. 
Definition 4.5. (see Figure 4.2) The elementary lamination $\lambda_{\gamma}$ corresponding to an arc $\gamma$ is the homotopy class of curves, contained in a neighbourhood of $\gamma$, defined as follows:

- if $\gamma$ is a chord connecting $p$ and $q$ then $\lambda_{\gamma}$ connects $p^{\prime}$ and $q^{\prime}$;

- if $\gamma$ is a radius tagged plain (resp. notched) starting from $p$ then $\lambda_{\gamma}$ starts from $p^{\prime}$ and winds counterclockwise (resp. clockwise) infinitely many times around the puncture.

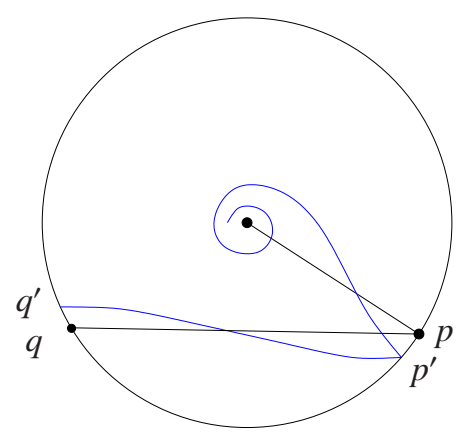

Figure 4.2: Examples of elementary laminations.

The shear coordinates of an elementary lamination $\lambda$ with respect to a triangulation $\Gamma$ are the integers in the $n$-tuple $\left(b_{\lambda, \gamma}^{\Gamma}\right)_{\gamma \in \Gamma}$ defined in terms of intersections between $\lambda$ and the unique quadrilateral in $\Gamma$ of which $\gamma$ is the diagonal.

More precisely assume, at first, that $\Gamma$ contains at most one notched radius; each segment of $\lambda$ cutting through the quadrilateral enclosing $\gamma$ as in Figure 4.3 contributes either +1 or -1 to $b_{\lambda, \gamma}^{\Gamma}$. All other crossings do not contribute. Note in particular that, if $\gamma$ is a radius of a digon then, to have have nonzero shear coordinate, a lamination as to "go around the puncture". When the digon has two non homotopic radii this means that the lamination has to intersect both of them; in the other case the lamination has to cross the dotted line joining the puncture to the boundary. We will continue to draw this dotted line whenever we have a digon with homotopic radii. Note also that flipping $\gamma$ interchanges positive and negative crossings.

To extend the definition to all possible triangulations it suffices to impose that, if $\Gamma^{\vee}$ is obtained from $\Gamma$ by changing all the tags at the puncture and $\lambda^{\vee}$ is obtained from $\lambda$ inverting its winding direction (if any), then for any $\gamma \in \Gamma$

$$
b_{\lambda \vee, \gamma \vee}^{\Gamma^{\vee}}=b_{\lambda, \gamma}^{\Gamma} .
$$

Given a triangulation $\Gamma$ let $\Lambda(\Gamma)=\left\{\lambda_{\gamma}\right\}_{\gamma \in \Gamma}$ be the multilamination associated to it, i.e. the collection of the elementary laminations corresponding to the arcs of $\Gamma$. Let $\widetilde{B}_{\Gamma}\left(\Gamma^{\prime}\right)$ be the extended $B$-matrix having top part $B\left(\Gamma^{\prime}\right)$ defined by $(4.1)$ and bottom part given by the shear coordinates of $\Lambda(\Gamma)$ with respect to $\Gamma^{\prime}$. 

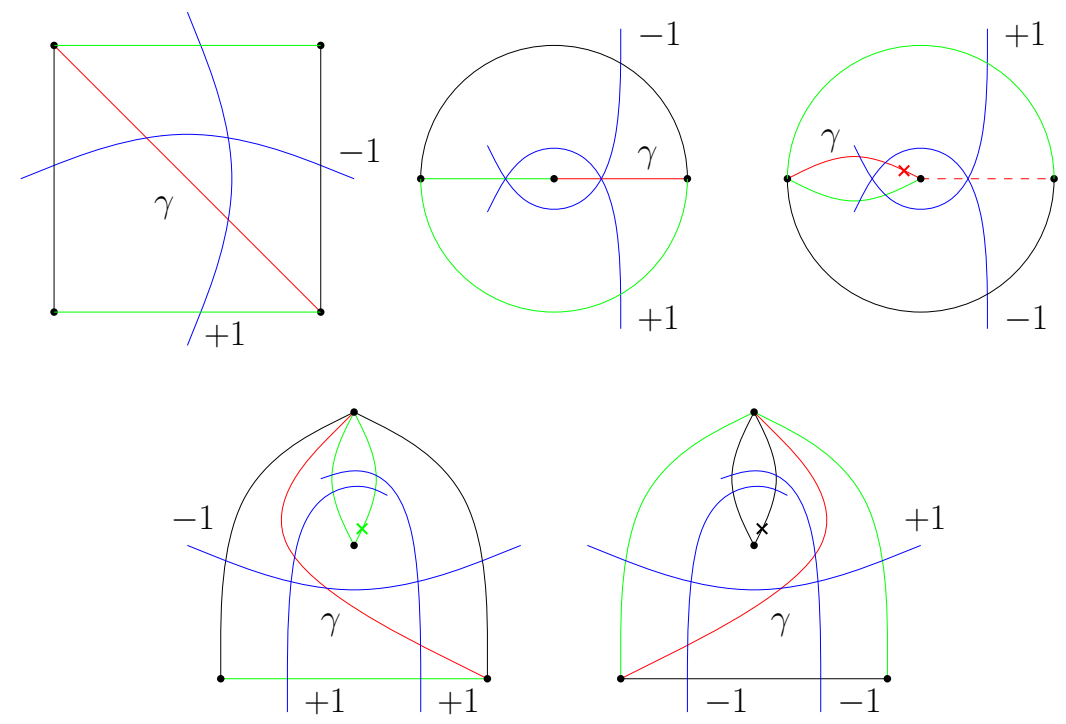

Figure 4.3: Intersections giving non-zero shear coordinates. The highlighted edges are those crossed by laminations $\lambda$ giving positive coordinates $b_{\lambda, \gamma}^{\Gamma}$.

Proposition 4.6 ([25, Proposition 16.3]). In the principal-coefficients cluster algebra $\mathcal{A}_{\mathbf{0}}(B(\Gamma))$ the extended exchange matrix corresponding to the triangulation $\Gamma^{\prime}$ is given by the above $\widetilde{B}_{\Gamma}\left(\Gamma^{\prime}\right)$.

We can now describe the sets $\mathcal{C}(B)$ and $\mathcal{D}(B)$. For the rest of this section fix a skewsymmetric integer matrix $B$ of type $A_{n}$ or $D_{n}$. Let $\Lambda_{0}=\left\{\lambda_{i}\right\}_{i \in I}$ be the multilamination corresponding to a triangulation $\Gamma_{0}=\left\{\gamma_{i}\right\}_{i \in I}$ of $S$ realizing $B$. In view of the last Proposition the set of $c$-vectors of the principal-coefficients cluster algebra $\mathcal{A}_{\bullet}(B)$ is

$$
\mathcal{C}(B)=\left\{c_{\gamma, \Gamma}:=\left(b_{\lambda_{i}, \gamma}^{\Gamma}\right)_{i \in I}\right\}
$$

as $\Gamma$ runs over all possible triangulations of $S$ and $\gamma$ is an $\operatorname{arc}$ in $\Gamma$. The parametrization of $\mathcal{C}(B)$ by pairs of arcs and triangulations is not one to one; indeed for any given $c$-vector there are in general many pairs $\gamma, \Gamma$ realizing it. We will see that $\Gamma$ can always be chosen to be bipartite (see Proposition 4.11).

As we already noted, in a cluster algebra coming from a surface, cluster variables are in bijection with tagged arcs. Their denominator vectors can be read directly from the surface: they are given in terms of their intersection pairing with the arcs of the initial triangulation.

Theorem 4.7 ([24, Theorem 8.6],[29, Theorem 3.4]). Let $\mathcal{A}_{\bullet}(B)$ be any cluster algebra of type $A_{n}$ or $D_{n}$ and let $\Gamma_{0}=\left\{\gamma_{i}\right\}_{i \in I}$ be a triangulation corresponding to $B$.

If $x_{\gamma}$ is the cluster variable corresponding to the tagged arc $\gamma$ then its d-vector is

$$
d_{\gamma}=\left(\left(\gamma_{i} \mid \gamma\right)\right)_{i \in I} .
$$


The set of non-initial $d$-vectors of $\mathcal{A}_{\bullet}(B)$ is therefore

$$
\mathcal{D}(B)=\left\{d_{\gamma}=\left(\left(\gamma_{i} \mid \gamma\right)\right)_{i \in I}\right\}
$$

as $\gamma$ runs over all arcs of $S$ not in $\Gamma_{0}$.

\subsection{Proof of Theorem 1.1}

We begin by providing an alternative and immediate proof of (1.3) for types $A_{n}$ and $D_{n}$.

Lemma 4.8. All the vectors in $\mathcal{C}(B)$ are sign-coherent.

Proof. By contradiction let $c_{\gamma, \Gamma}$ be a $c$-vector that is not sign-coherent i.e. there are two elementary laminations in $\Lambda_{0}$, say $\lambda_{i}$ and $\lambda_{j}$ such that $b_{\lambda_{i}, \gamma}^{\Gamma}>0$ and $b_{\lambda_{j}, \gamma}^{\Gamma}<0$.

Assume at first that $\Gamma$ contains at most one notched arc. From Figure 4.3 it is clear that $\lambda_{i}$ and $\lambda_{j}$ intersect and if they both spiral to the puncture then they do not come from homotopic radii. This is in contradiction with the hypothesis that $\Lambda_{0}$ came from a triangulation of $S$ : the intersection pairing of the arcs corresponding to $\lambda_{i}$ and $\lambda_{j}$ is positive.

The results extends immediately to all the possible triangulation if we observe that changing the windings of all the laminations spiraling to the puncture does not affect the intersection relations among elements of $\Lambda_{0}$.

Note that, if $c_{\gamma, \Gamma}$ is a $c$-vector and $\Gamma^{\prime}$ is the triangulation obtained from $\Gamma$ by flipping $\gamma$ into $\gamma^{\prime}$, then

$$
c_{\gamma, \Gamma}=-c_{\gamma^{\prime}, \Gamma^{\prime}}
$$

From now on we concentrate on the set $\mathcal{C}_{+}(B)$ of positive $c$-vectors of $\mathcal{A}_{\bullet}(B)$.

Lemma 4.9. The weighted diagram of any positive c-vector in $\mathcal{A}_{\bullet}(B)$ is connected.

Proof. By contradiction assume that the weighted diagram of $c_{\gamma, \Gamma}$ has two disjoint components. Let $i$ be a node in one of them and $j$ a node in the other such that they are at minimal distance in $X(B)$. By hypothesis $i$ and $j$ are not adjacent. Let $\lambda_{i}$ and $\lambda_{j}$ be the corresponding elementary laminations in $\Lambda_{0}$.

Three cases are possible (in type $A_{n}$ only the last one occurs).

1. If $\lambda_{i}$ and $\lambda_{j}$ have two endpoints in common then they spiral to the puncture in opposite directions. In this case, since both $b_{\lambda_{i}, \gamma}^{\Gamma} \neq 0$ and $b_{\lambda_{j}, \gamma}^{\Gamma} \neq 0$, the arc $\gamma$ cannot be incident to the puncture. The multilamination $\Lambda_{0}$ contains then a bigon enclosing $\lambda_{i}$ and $\lambda_{j}$; at least one side of this bigon (say $\lambda_{k}$ ) crosses positively the quadrilateral enclosing $\gamma$.

2. If $\lambda_{i}$ and $\lambda_{j}$ share exactly one endpoint, since $i$ and $j$ are not adjacent, there are two possible configurations. If there is no other lamination sharing that endpoint then they both spiral to the puncture and they are enclosed in a bigon; at least one side of this bigon (again say $\lambda_{k}$ ) intersects positively the quadrilateral enclosing $\gamma$. Otherwise at least one lamination $\lambda_{k}$ among those sharing the same endpoint is such that $b_{\lambda_{k}, \gamma}^{\Gamma}>0$. 
3. Finally if $\lambda_{i}$ and $\lambda_{j}$ do not share any endpoint then there is at least one lamination $\lambda_{k}$ starting from one of those four points, lying in between $\lambda_{i}$ and $\lambda_{j}$ and crossing positively the quadrilateral that encloses $\gamma$ (otherwise such an elementary lamination could be added to $\Lambda_{0}$ in contradiction to the assumption that the multilamination corresponds to a triangulation).

In all of the cases there is a vertex $k$ in between $i$ and $j$ such that the $k$-th component of $c_{\gamma, \Gamma}$ is non-zero in contradiction with the assumption of minimal distance between $i$ and $j$.

Proposition 4.10. In types $A_{n}$ and $D_{n}$ we have

$$
\mathcal{C}_{+}(B) \subset \mathcal{V}(B)
$$

Proof. We deal first with type $A_{n}$. It is clear that, having no puncture, any lamination $\lambda \in \Lambda_{0}$ can intersect any given arc $\gamma$ at most once so $b_{\lambda, \gamma}^{\Gamma} \in\{0,1\}$. In view of Proposition 3.1 it suffices to show that no $c$-vector can have a triangle in its weighted diagram. But this follows directly from the fact that, since $S$ has no puncture, at least one of the sides of each triangle in $\Lambda_{0}$ does not intersect any given arc $\gamma$.

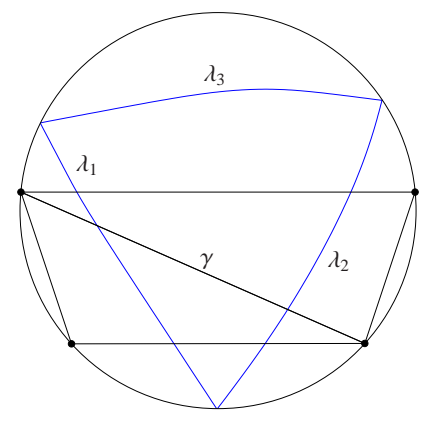

Figure 4.4: Any triangle in a lamination of a surface of type $A_{n}$ intersects at most twice any $\operatorname{arc} \gamma$.

For type $D_{n}$ the proof proceeds by case analysis. We need first some considerations. In view of condition (4.2) we can assume that the quadrilateral enclosing $\gamma$ is one of those in Figure 4.3.

Note that, given a multilamination $\Lambda_{0}$ coming from a triangulation, a once punctured disk can be decomposed into pieces: it will contain exactly one piece in which all the elementary laminations spiral to the puncture (one of the five in Figure 4.5); all the other pieces, if any, will contain only elementary laminations corresponding to chords. Any such piece can only be glued to the one containing the puncture as shown in Figure 4.6.

Any elementary lamination of $\Lambda_{0}$ not corresponding to a glued edge will be contained, up to a small neighbourhood of one endpoint, in exactly one piece in this decomposition. This implies that any given piece must contain at least a section of $\gamma$ and of two opposite 


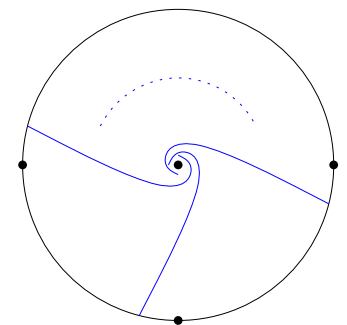

Plain $n$-gon $(n \geq 3)$

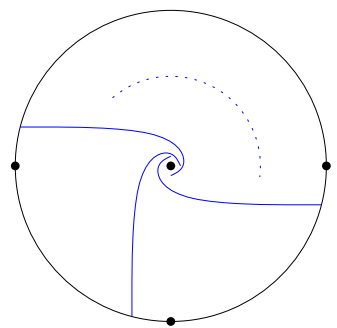

Notched $n$-gon $(n \geq 3)$

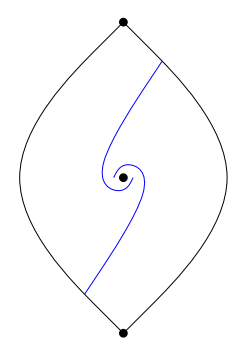

Plain digon

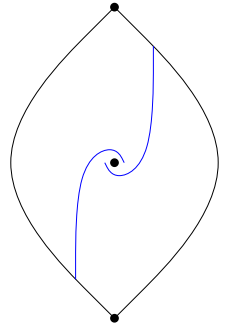

Notched digon

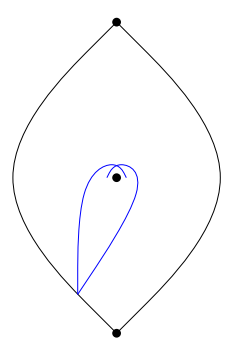

Folded digon

Figure 4.5: Multilaminations with all elementary laminations spiralling to the puncture.

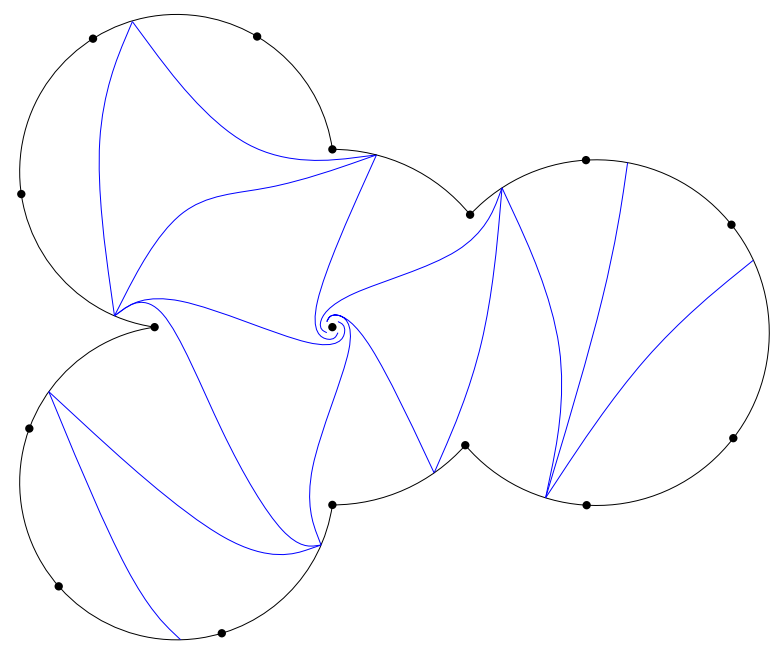

Figure 4.6: Example of a decomposition of a surface of type $D_{n}$ according to a multilamination. 
sides of the quadrilateral enclosing $\gamma$ in order for any of the laminations it contains to give rise to a positive coordinate. In particular a quadrilateral of a triangulation can intersect non trivially at most three pieces in this decomposition.

We need therefore to consider all the possible ways a quadrilateral from Figure 4.3 can be fitted into a surface with at most three pieces. This is a straightforward but tedious check; a complete analysis of the various cases (87 nontrivial cases in total) is contained in Appendix A.

To connect $\mathcal{C}_{+}(B)$ with $\mathcal{D}(B)$ let us improve on the parametrization of $c$-vectors of $\mathcal{A}_{\bullet}(B)$. A triangulation $\Gamma$ of $S$ is said to be bipartite if every node of the corresponding quiver is either a sink or a source. Note that, since in finite type any chordless cycle must be oriented ([2] Theorem 1.2), bipartite triangulations correspond to bipartite orientations of the Dynkin diagram of the given type.

Not every quadrilateral can appear in a bipartite triangulation; indeed it is clear from the assignment (4.1) that the only allowed one are those in Figure 4.7. Moreover, given any such quadrilateral, there exists a unique bipartite triangulation in which it appears.
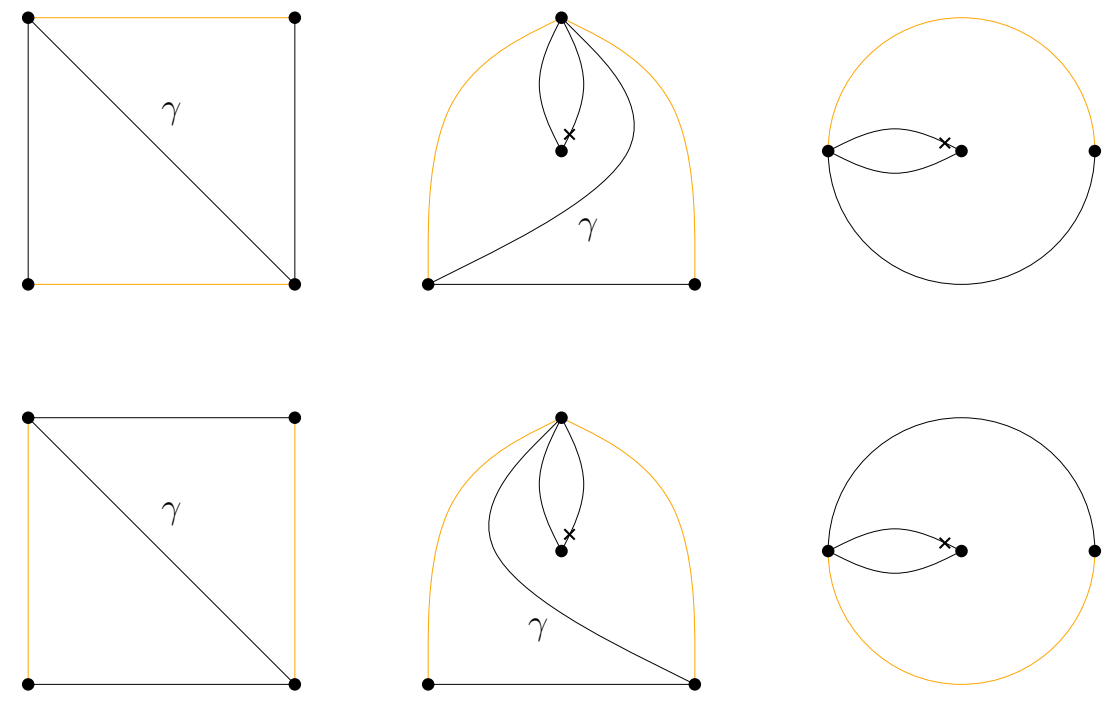

Figure 4.7: The only quadrilaterals that can appear in a bipartite triangulation of a surface $S$. The edges on the boundary of $S$ are highlighted. When the quadrilateral is a digon any of the radii can be the diagonal $\gamma$.

Let $\mathcal{C}_{+}^{b}(B)$ be the subset of $\mathcal{C}_{+}(B)$ consisting of $c$-vectors $c_{\gamma, \Gamma}$ such that $\Gamma$ is bipartite.

Proposition 4.11. In types $A_{n}$ and $D_{n}$

$$
\mathcal{C}_{+}^{b}(B)=\mathcal{C}_{+}(B)
$$


Proof. Let $c_{\gamma, \Gamma}$ be any element of $\mathcal{C}_{+}(B)$; we need to show that there exists a bipartite triangulation $\Gamma^{\prime}$ and an arc $\gamma^{\prime} \in \Gamma^{\prime}$ such that $c_{\gamma, \Gamma}=c_{\gamma^{\prime}, \Gamma^{\prime}}$.

Let $\Lambda_{0}$ be the multilamination associated to $B$. In view of the observation we just made we only need to construct a quadrilateral like those in Figure 4.7 having the same intersections with $\Lambda_{0}$ that $\Gamma$ does: this will automatically determine the bipartite triangulation we are after.

We concentrate first on type $A_{n}$. The idea is simple: pick a leaf in the support of $c_{\gamma, \Gamma}$ and let $\lambda$ be the corresponding elementary lamination in $\Lambda_{0}$. Since $\lambda$ is the "last" lamination intersecting the quadrilateral enclosing $\gamma$ positively it must belong to a triangle in $\Lambda_{0}$ such that the other two lamination composing it do not give rise to positive shear coordinates. Let $p^{\prime}$ be the only vertex of the triangle that is not incident to $\lambda$. We can replace the original quadrilateral with one having the two marked points closest to $p^{\prime}$ as vertices: all the shear coordinates will be unchanged. We can than conclude by applying the same procedure to the other leaf (cf. Figure 4.8).

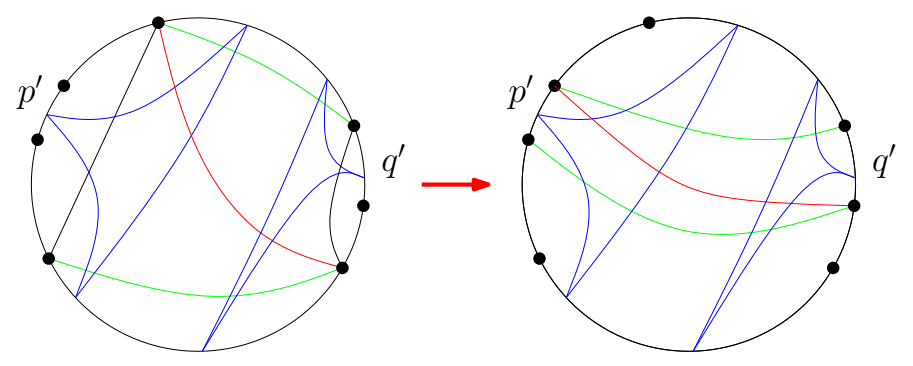

Figure 4.8: The reduction of a quadrilateral to a bipartite quadrilateral in type $A_{n}$. The quadrilateral on the right give raise to the same shear coordinates produced by the quadrilateral on the left and determines uniquely a bipartite triangulation.

This is sufficient in type $A_{n}$ but not in general in type $D_{n}$ : we need to deal with folded quadrilaterals as well. The replacement to be performed depends both on $\Lambda_{0}$ and $\gamma$ but it is straightforward from the pictures. The general procedure is shown in Figure 4.9. The reduction is in two steps: first we apply the same strategy of type $A_{n}$ to have the correct amount of edges of the quadrilateral on the boundary of the surface. Then, if needed, we replace the quadrilateral we obtain with one from Figure 4.7. The precise case analysis is again in Appendix A; there we provide, for each possible quadrilateral and for each multilamination an explicit replacement.

In analogy with the definition above let $\mathcal{D}^{b}(B)$ be the subset of all the non-initial $d$-vectors corresponding to cluster variables appearing in bipartite seeds of $\mathcal{A}_{\bullet}(B)$. Since any arc on $S$ appears in a bipartite triangulation, in types $A_{n}$ and $D_{n}$ we have

$$
\mathcal{D}^{b}(B)=\mathcal{D}(B)
$$

Remark 4.12. The above equality, together with Proposition 4.11, prove Theorem 1.4 for cluster algebras of types $A_{n}$ and $D_{n}$. 


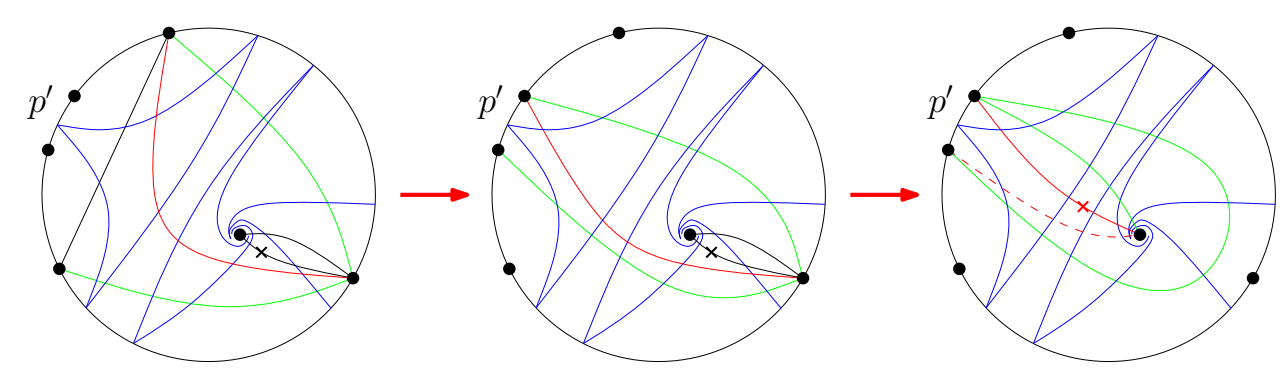

Figure 4.9: An example of the reduction of a quadrilateral to a bipartite quadrilateral in type $D_{n}$.

Proposition 4.13. In types $A_{n}$ and $D_{n}$

$$
\mathcal{C}_{+}(B)=\mathcal{D}(B)
$$

Proof. In view of the above reductions it suffices to show that

$$
\mathcal{C}_{+}^{b}(B)=\mathcal{D}^{b}(B)
$$

As before let $\Gamma_{0}=\left\{\gamma_{i}\right\}_{i \in I}$ be the triangulation corresponding to $B$ and $\Lambda_{0}=\left\{\lambda_{i}\right\}_{i \in I}$ the associated multilamination. In view of Theorem 4.7 and Definition 4.3 all the vectors in $\mathcal{D}^{b}(B)$ have non-negative components.

Let $\gamma$ be any arc not in $\Gamma_{0}$ and consider the $d$-vector $d_{\gamma}$; we need to distinguish three cases (cf. Figure 4.10) depending on the endpoints of $\gamma$ (call them $p$ and $q$ ).

- If both $p$ and $q$ are on the boundary of $S$ and they are not adjacent then there are two other marked points $r$ and $s$ such that $p^{\prime}$ is contained on the boundary segment $p r$ and $q^{\prime}$ is contained in the boundary segment $q s$. Let $\gamma^{\prime}$ be the diagonal $r s$ of the quadrilateral prqs and complete the quadrilateral to a bipartite triangulation $\Gamma^{\prime}$. We have $d_{\gamma}=c_{\gamma^{\prime}, \Gamma^{\prime}}$. Note that if $S$ is of type $A_{n}$ this is the only possible case.

- It both $p$ and $q$ are on the boundary of $S$ and they are adjacent then we can assume (up to relabeling) that $q^{\prime}$ lies on the boundary segment $q p$. Let $r$ be such that $p^{\prime}$ lies on the boundary segment $p r$. Let $\gamma^{\prime}$ be the diagonal $p r$ of the folded quadrilateral having vertices $q, p, r$, and the puncture and having two homotopic radii starting at $p$; Let $\Gamma^{\prime}$ be the bipartite triangulation containing this quadrilateral. We have again $d_{\gamma}=c_{\gamma^{\prime}, \Gamma^{\prime}}$.

- If one of the endpoints of $\gamma$ (say $q$ to fix ideas) is the puncture then let $r$ be the marked point such that $p^{\prime}$ lies between $p$ and $r$. Let $\Gamma^{\prime}$ be the bipartite triangulation containing the digon with vertices $p$ and $r$, enclosing the puncture, and such that its radii both start from $r$. If $\gamma^{\prime}$ is the radius with tagging opposite to the tagging of $\gamma$ then $d_{\gamma}=c_{\gamma^{\prime}, \Gamma^{\prime}}$. 

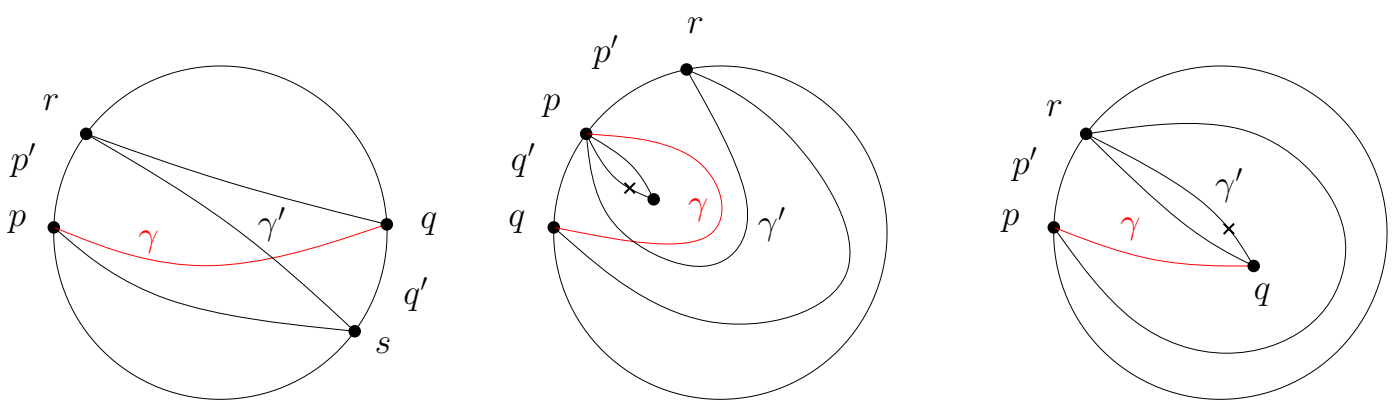

Figure 4.10: The three possible cases of Proposition 4.13.

Conversely let $c_{\gamma^{\prime}, \Gamma^{\prime}}$ in $\mathcal{C}_{+}^{b}(B)$. The quadrilateral of $\Gamma^{\prime}$ enclosing $\gamma^{\prime}$ will be exactly one of those constructed above (they are all bipartite). Choosing $\gamma$ to be the corresponding arc we get $d_{\gamma}=c_{\gamma^{\prime}, \Gamma^{\prime}}$.

We thank Andrei Zelevinsky for providing the idea of using the "bipartite belt" in the above proof.

The following Proposition concludes the proof of Theorem 1.1 for types $A_{n}$ and $D_{n}$.

Proposition 4.14. In types $A_{n}$ and $D_{n}$ we have

$$
\mathcal{V}(B) \subset \mathcal{D}(B)
$$

Proof. Let $\Gamma_{0}=\left\{\gamma_{i}\right\}_{i \in I}$ be a triangulation realizing $B$ and let $v=\left(v_{i}\right)_{i \in I}$ be any element in $\mathcal{V}(B)$.

In type $A_{n}$ it is clear how to construct an arc $\gamma$ crossing exactly one time all the arcs $\gamma_{j}$ such that $v_{j} \neq 0$ : suppose $i$ is a leaf in the weighted diagram; the arc $\gamma_{i}$ corresponding to it belongs to two triangles. One of them is such that the nodes corresponding to the other two arcs forming it do not belong to the support of the weighted diagram. The arc $\gamma$ we are looking for starts from the vertex of this triangle opposed to $\gamma_{i}$. It crosses then in sequence all the $\operatorname{arcs} \gamma_{j}$ such that $v_{j} \neq 0$ and terminates in the vertex opposite to the arc corresponding to the other leaf.

In type $D_{n}$ the procedure is slightly more involved and depends on the initial triangulation $\Gamma_{0}$ but follows the same basic idea. Suppose at first that $\Gamma_{0}$ does not contain a digon with two homotopic radii. The same procedure described for type $A_{n}$ works verbatim for diagrams I, IV, and VIII in Figure 3.7: the only thing to note is that instead of a leaf we might have to take one of the nodes in the left triangle (for IV we cannot use the two rightmost leaves). For diagrams II and VI we need a small fix: $\gamma$ starts from the vertex opposite to the arc corresponding to the leftmost leaf and ends at the puncture; its tagging is the opposite of the tagging of the radii in $\Gamma_{0}$. For diagrams III and VII we repeat the same argument using the leftmost leaf and the leftmost node with multiplicity 2. Diagrams like $\mathrm{V}$ cannot be embedded in a $X(B)$ if $\Gamma_{0}$ does not have a digon with two homotopic radii in it. 
If $\Gamma_{0}$ contains a digon with two homotopic radii then the only diagrams that can arise are I, II, III, IV, and V. For V the procedure is the same as the one for type $A_{n}$, we just need to cross both the radii of the digon. For diagrams III and IV the procedure is identical to the above. For diagrams like II $\gamma$ starts from the vertex opposite to the arc corresponding to the leftmost leaf and ends in the vertex of the digon not adjacent to the radii. For diagrams like I we need to distinguish two cases: if one of the leaves corresponds to a radius then the corresponding endpoint of $\gamma$ is the puncture and its tagging is the opposite of the one of that radius. Otherwise we proceed as in type $A_{n}$.

\section{Types $B_{n}$ and $C_{n}$ : the folding method}

Building on the results of last section we will now prove Theorem 1.1 for types $B_{n}$ and $C_{n}$. In order to do so we will realize any principal coefficients cluster algebra of type $B_{n}$ (respectively $C_{n}$ ) as a subquotient of an appropriate cluster algebra of type $D_{n+1}$ (respectively $A_{2 n-1}$ ) with principal coefficients.

\subsection{Folding of cluster algebras with trivial coefficients}

The construction, for the coefficient-free case, was explained in [21]. Since we need to generalize it to work with principal coefficients later on let us begin by recalling in some details its main features.

Let $B=\left(b_{i j}\right)_{i, j \in I}$ be a skew-symmetrizable integer matrix and $\sigma$ a permutation of $I$.

Definition 5.1. A permutation $\sigma$ is an automorphism of $B$ if, for any $i$ and $j$ in $I$,

$$
b_{\sigma(i) \sigma(j)}=b_{i j} .
$$

An automorphism of $B$ is said to be admissible if, for any $i_{1}$ and $i_{2}$ in the same $\sigma$-orbit $\bar{\imath}$ and for any $j$ in $I$,

$$
\begin{gathered}
b_{i_{1}, j} b_{i_{2} j} \geqslant 0 \\
b_{i_{1}, i_{2}}=0 .
\end{gathered}
$$

An easy computation shows that, if $\sigma$ is an admissible automorphism of $B$ and $k_{1}$ and $k_{2}$ are two points in the same $\sigma$-orbit $\bar{k}$, the mutations $\mu_{k_{1}}$ and $\mu_{k_{2}}$ commute; that is

$$
\mu_{k_{1}} \circ \mu_{k_{2}}(B)=\mu_{k_{2}} \circ \mu_{k_{1}}(B) \text {. }
$$

Indeed $\mu_{k_{2}}\left(\mu_{k_{1}}\left(b_{i j}\right)\right)$ is either $-b_{i j}$, if at least one among $i$ and $j$ is in $\left\{k_{1}, k_{2}\right\}$, or

$$
b_{i j}+b_{i k_{1}}\left[b_{k_{1} j}\right]_{+}+\left[-b_{i k_{1}}\right]_{+} b_{k_{1} j}+b_{i k_{2}}\left[b_{k_{2} j}\right]_{+}+\left[-b_{i k_{2}}\right]_{+} b_{k_{2} j}
$$

otherwise. Those expressions are clearly independent on the order in which $\mu_{k_{1}}$ and $\mu_{k_{2}}$ are applied. It makes therefore sense to define orbit-mutations as the compositions

$$
\mu_{\bar{k}}^{\sigma}:=\prod_{t \in \bar{k}} \mu_{t}
$$

THE ELECTRONiC JOURnAl OF COMBinAtorics 21(1) (2014), \#P1.3 
Repeating the same reasoning we get

$$
\mu_{\bar{k}}^{\sigma}\left(b_{i j}\right)= \begin{cases}-b_{i j} & \text { if } i \text { or } j \in \bar{k} \\ b_{i j}+\sum_{t \in \bar{k}}\left(b_{i t}\left[b_{t j}\right]_{+}+\left[-b_{i t}\right]_{+} b_{t j}\right) & \text { otherwise }\end{cases}
$$

Note that, given a $\sigma$-orbit $\bar{k}$, the permutation $\sigma$ is always an automorphism of $\mu_{\bar{k}}^{\sigma}(B)$ but it need not be admissible; in particular condition (5.2) may be violated.

Definition 5.2. An admissible automorphism $\sigma$ of $B$ is said to be stable if, for any finite sequence of $\sigma$-orbits $\overline{k_{1}}, \ldots \overline{k_{\ell}}$, it is an admissible automorphism of

$$
\mu \frac{\sigma}{k_{\ell}} \circ \cdots \circ \mu \frac{\sigma}{k_{1}}(B)
$$

Proposition 5.3 ([21, Proposition 2.22]). If the Cartan counterpart of B is a simply-laced finite type then any admissible automorphism of $B$ is stable.

Remark 5.4. We will need the following incarnations of Proposition 5.3:

1. $B$ has Cartan counterpart of type $A_{2 n-1}$ and, using the standard labeling of the nodes of the associated Dynkin diagram,

$$
\sigma:=\prod_{i=1}^{n}(i, 2 n-i)
$$

is an admissible automorphism of $B$.

2. $B$ has Cartan counterpart of type $D_{n+1}$ and, again in the standard labeling,

$$
\sigma=(n, n+1)
$$

is an admissible automorphism of $B$.

Given a skew-symmetrizable integer matrix $B$ and a (stable) admissible automorphism $\sigma$ we can define a folded matrix $\pi(B):=\bar{B}=\left(b_{\bar{\imath} \jmath}\right)$, as $\bar{\imath}$ and $\bar{\jmath}$ vary over all the $\sigma$-orbits, by setting

$$
b_{\bar{\imath}}:=\sum_{s \in \bar{\imath}} b_{s j} .
$$

In view of condition (5.1) the value of $b_{\bar{\imath} \jmath}$ does not depend on the choice of a representative of $\bar{\jmath}$. The folded matrix $\pi(B)$ is itself skew-symmetrizable (see [21, Lemma 2.5]).

The key point here is this: if $\sigma$ is a stable admissible automorphism of $B$ then for any $\sigma$-orbit $\bar{k}$

$$
\pi\left(\mu_{\bar{k}}^{\sigma}(B)\right)=\mu_{\bar{k}}(\pi(B))
$$

thanks to condition (5.2) (see [21, Theorem 2.24]).

We will use the following obvious converse stating the existence of "unfolding" for the matrices we are interested into. 
Proposition 5.5. Let $\bar{B}^{\prime}$ be any matrix in the same mutation class of a matrix $\bar{B}$ obtained by folding from a skew-symmetrizable matrix $B$ with a stable admissible automorphism $\sigma$. There exist a matrix $B^{\prime}$ and a sequence of $\sigma$-orbits $\overline{k_{1}}, \ldots, \overline{k_{\ell}}$ such that

1. $\mu_{\overline{k_{\ell}}}^{\sigma} \circ \cdots \circ \mu_{\overline{k_{1}}}^{\sigma}(B)=B^{\prime}$

2. $\bar{B}^{\prime}=\overline{B^{\prime}}$.

The folding map can be extended to a morphism of algebras as follows. Fix an initial $B$-matrix $B$ and a stable admissible automorphism $\sigma$. Let

$$
\left(B,\left\{x_{i}\right\}_{i \in I}\right)
$$

be the initial cluster of the coefficient-free cluster algebra $\mathcal{A}_{0}(B)$. Write $\mathcal{A}_{0}^{\sigma}(B)$ for the subalgebra of $\mathcal{A}_{0}(B)$ generated by all the clusters reachable from the initial one by a sequence of orbit mutations.

Let $\mathcal{A}_{0}(\bar{B})$ be the coefficient-free cluster algebra with initial $B$-matrix $\pi(B)=\bar{B}$ and initial cluster variables $\left\{x_{\bar{\imath}}\right\}_{\bar{\imath} \in I / \sigma}$. The assignment

$$
\pi\left(x_{i}\right):=x_{\bar{\imath}}
$$

extends to a surjective map

$$
\pi: \mathcal{A}_{0}^{\sigma}(B) \longrightarrow \mathcal{A}_{0}(\bar{B}) .
$$

The algebra $\mathcal{A}_{0}(\bar{B})$ is the quotient of $\mathcal{A}_{0}^{\sigma}(B)$ by the ideal generated by the relations

$$
x_{i}=x_{\sigma(i)} .
$$

Moreover, and this is the key point in the construction, the map $\pi$ preserves the cluster structure: seeds of $\mathcal{A}_{0}^{\sigma}(B)$ are mapped to seeds of $\mathcal{A}_{0}(\bar{B})$.

Combining the above observation with Remark 5.4 we get the following statement.

Proposition 5.6. Any matrix of cluster type $B_{n}$ (respectively $C_{n}$ ) is the image $\pi(B)$ of a matrix $B$ of cluster type $D_{n+1}$ (respectively $A_{2 n-1}$ ) with automorphism $\sigma$ from Remark 5.4. The coefficient-free cluster algebra $\mathcal{A}_{0}(\bar{B})$ is the quotient of a subalgebra of $\mathcal{A}_{0}(B)$ by an ideal preserving the cluster structure. In particular any exchange matrix of $\mathcal{A}_{0}(\bar{B})$ is the folding of some exchange matrix of $\mathcal{A}_{0}(B)$.

\subsection{Proof of Propositions 3.2 and 3.3.}

The results just summarized are enough to describe the sets $\mathcal{X}\left(B_{n}\right)$ and $\mathcal{X}\left(C_{n}\right)$.

Proof of Proposition 3.2. In view of Proposition 5.6 any element of $\mathcal{X}\left(B_{n}\right)$ can be obtained by folding an element of $\mathcal{X}\left(D_{n+1}\right)$. On the other hand not every diagram from Figure 3.6 can be folded: we know that any chordless cycle in such a diagram corresponds to a cyclically oriented chordless cycle in the quiver $Q(B)$ associated to it (see [27, 2]). By 
definition of admissible automorphism all the vertices in the only non-trivial orbit of $\sigma$ must be not adjacent and must be connected to all the other adjacent vertices in the same way. This forces us to conclude that diagrams (c) and (d) cannot be folded.

The diagrams of Figure 3.2 are thus the folding of diagrams (a) and (b) from Figure 3.6.

Proof of Proposition 3.3. In view of Proposition 5.6 diagrams in $\mathcal{X}\left(C_{n}\right)$ are obtained by folding elements of $\mathcal{X}\left(A_{2 n-1}\right)$. The only requirement a diagram must satisfy to be folded is to be symmetric with respect to the only fixed point of $\sigma$ from Remark 5.4.

\subsection{Folding of $c$-vectors}

In order to consider $c$-vectors we need to extend the above construction to cluster algebras with principal coefficients. We take inspiration from the following example.

Example 5.7. Let $\mathcal{A}_{\bullet}(B)$ be the cluster algebra of type $D_{4}$ with principal coefficients at the initial cluster given by

$$
B=\left(\begin{array}{cccc}
0 & -1 & 0 & 0 \\
1 & 0 & -1 & -1 \\
0 & 1 & 0 & 0 \\
0 & 1 & 0 & 0
\end{array}\right)
$$

$B$ is invariant under permutation $\sigma=(34)$ and has $b_{34}=0$. Moreover the mutations in directions 3 and 4 commute; that is

$$
\mu_{3} \circ \mu_{4}(B)=\mu_{4} \circ \mu_{3}(B) .
$$

Let $\mathcal{A}_{\bullet}^{\sigma}(B)$ be the subalgebra of all the clusters reachable from the initial one by any sequence of the mutations $\mu_{1}, \mu_{2}$, and $\mu_{3} \circ \mu_{4}$. All the $B$-matrices in it have $b_{34}=0$.

The permutation $\sigma$ acts on the set of clusters of $\mathcal{A}_{\bullet}(B)$ by relabeling:

$$
\sigma\left(x_{i}\right):=x_{\sigma(i)} \quad \text { and } \quad \sigma\left(y_{i}\right):=y_{\sigma(i)}
$$

Let $\mathcal{I}$ be the ring ideal of $\mathcal{A}_{\bullet}^{\sigma}(B)$ generated by the relations

$$
x_{3}=x_{4} \quad \text { and } \quad y_{3}=y_{4}
$$

The quotient $\mathcal{A}_{\bullet}^{\sigma}(B) / \mathcal{I}$ is a cluster algebra of type $B_{3}$ with principal coefficients at the initial cluster given by $\pi(B)$. Under the projection map clusters of $\mathcal{A}_{\bullet}^{\sigma}(B)$ are mapped to clusters of $\mathcal{A}_{\bullet}^{\sigma}(B) / \mathcal{I}$. Moreover exchange relations in the quotient come from exchange relations of $\mathcal{A}_{\bullet}(B)$.

For any skew-symmetrizable integer matrix $B$ endowed with a stable admissible automorphism $\sigma$ let $\mathcal{A}_{\bullet}(B)$ and $\mathcal{A}_{\bullet}(\bar{B})$ be the cluster algebras with principal coefficients respectively at $B$ and $\bar{B}=\pi(B)$. Let $\mathcal{A}_{\bullet}^{\sigma}(B)$ be the subalgebra of $\mathcal{A}_{\bullet}(B)$ generated by all clusters reachable from the initial one using orbit-mutations. 
In view of the above example it is natural to define folding for a $c$-vector $c=\left(c_{i}\right)_{i \in I}$ of $\mathcal{A}_{\bullet}^{\sigma}(B)$ componentwise as follows:

$$
c_{\bar{\imath}}:=\sum_{s \in \bar{\imath}} c_{s}
$$

However the correctness of this definition is not so obvious because the tropicalization map (2.8) and folding are not compatible in general. Let us clarify the condition required to guarantee that (5.6) is well-posed.

Note that if $C$ and $C^{\prime}$ are two coefficient matrices of $\mathcal{A}_{\bullet}^{\sigma}(B)$ connected by a single orbit-mutation $\mu_{\bar{k}}$ then it follows directly from having assumed $\sigma$ to be a stable admissible automorphism of $B$ that:

$$
c_{i j}^{\prime}= \begin{cases}-c_{i j} & \text { if } j \in \bar{k} \\ c_{i j}+\sum_{t \in \bar{k}}\left(c_{i t}\left[b_{t j}\right]_{+}+\left[-c_{i t}\right]_{+} b_{t j}\right) & \text { otherwise. }\end{cases}
$$

From (5.7) we get an important observation: all the $C$-matrices in $\mathcal{A}_{\bullet}^{\sigma}(B)$ are such that

$$
c_{\sigma(i) \sigma(j)}=c_{i j} .
$$

Indeed the property holds for the initial $C$-matrix and we can use the admissibility of $\sigma$ to propagate it.

We introduce the folded $C$-matrix $\bar{C}$ for a $C$-matrix $C=\left(c_{i j}\right)_{i j \in I}$ of $\mathcal{A}_{\bullet}^{\sigma}(B)$ as

$$
c_{\bar{\imath}}:=\sum_{s \in \bar{\imath}} c_{s j}
$$

Note that (5.9) is independent of the choice of a representative of $\bar{\jmath}$ due to the symmetry (5.8).

Proposition 5.8. Let $B$ be any skew-symmetrizable integer matrix and let $\sigma$ be a stable admissible automorphism of $B$. The matrix $\bar{C}$ satisfies the recursion relation

$$
c_{\overline{\imath \jmath}}^{\prime}= \begin{cases}-c_{\overline{\imath \jmath}} & \text { if } \bar{\jmath}=\bar{k} \\ c_{\bar{\imath} \bar{\jmath}}+c_{\bar{\imath} \bar{k}}\left[b_{\bar{k} \bar{\jmath}}\right]_{+}+\left[-c_{\bar{\imath} \bar{k}}\right]_{+} b_{\bar{k} \bar{\jmath}} & \text { otherwise }\end{cases}
$$

if and only if the following condition holds: for any $i$ and $j$ the sign of $c_{s j}$ is independent of the choice of representative $s \in \bar{\imath}$.

Proof. It suffices to establish the proposition for a single mutation; if $\bar{\jmath}=\bar{k}$ our claim is trivial so we can assume $j \notin \bar{k}$. On the one hand we can rewrite (5.10) as

$$
c_{\bar{\imath} \bar{l}}^{\prime}=c_{\bar{\imath} \bar{\jmath}}+c_{\bar{\imath} \bar{k}}\left[b_{\bar{k} \bar{\jmath}}\right]_{+}+\left[-c_{\bar{\imath} \bar{k}}\right]_{+} b_{\bar{k} \bar{\jmath}}=\sum_{s \in \bar{\imath}} c_{s j}+\sum_{s \in \bar{\imath}} c_{s k}\left[\sum_{t \in \bar{k}} b_{t j}\right]_{+}+\left[-\sum_{s \in \bar{\imath}} c_{s k}\right]_{+} \sum_{t \in \bar{k}} b_{t j} .
$$


On the other hand

$$
c_{\overline{\imath \jmath}}^{\prime}=\sum_{s \in \bar{\imath}} c_{s j}^{\prime}=\sum_{s \in \bar{\imath}}\left(c_{s j}+\sum_{t \in \bar{k}}\left(c_{s t}\left[b_{t j}\right]_{+}+\left[-c_{s t}\right]_{+} b_{t j}\right)\right) .
$$

Therefore the recursion 5.10 is satisfied if and only if

$$
\sum_{s \in \bar{\imath}} c_{s k}\left[\sum_{t \in \bar{k}} b_{t j}\right]_{+}=\sum_{s \in \bar{\imath}} \sum_{t \in \bar{k}} c_{s t}\left[b_{t j}\right]_{+}
$$

and

$$
\left[-\sum_{s \in \bar{\imath}} c_{s k}\right]_{+} \sum_{t \in \bar{k}} b_{t j}=\sum_{s \in \bar{\imath}} \sum_{t \in \bar{k}}\left[-c_{s t}\right]_{+} b_{t j} .
$$

The first condition is guaranteed by the admissibility of $\sigma$; indeed we get

$$
\sum_{s \in \bar{\imath}} \sum_{t \in \bar{k}} c_{s k}\left[b_{t j}\right]_{+}=\sum_{s \in \bar{\imath}} \sum_{t \in \bar{k}} c_{s t}\left[b_{t j}\right]_{+}
$$

which is true by a simple change of summation index using (5.8).

Similarly the second condition is equivalent to

$$
\sum_{s \in \bar{\imath}} \sum_{t \in \bar{k}}\left[-c_{s k}\right]_{+} b_{t j}=\sum_{s \in \bar{\imath}} \sum_{t \in \bar{k}}\left[-c_{s t}\right]_{+} b_{t j} .
$$

if and only if the sign of $c_{s k}$ is independent on the choice of representative $s \in \bar{l}$.

In our situation the condition of Proposition 5.8 is satisfied by the sign-coherence property of $c$-vectors established in Lemma 4.8 or, more generally for skew-symmetric $B$-matrices, explained in Section 2. Thus definition (5.6) is well-posed in our case.

It is worth noticing at this point that the folding map (5.6) sends the identity matrix to the identity matrix: as one might expect the image of the initial cluster of $\mathcal{A}_{\bullet}(B)$ is the initial cluster of $\mathcal{A}_{\bullet}(\bar{B})$.

Corollary 5.9. Let $\bar{B}$ be any skew-symmetrizable matrix of cluster type $B_{n}$ (respectively $C_{n}$ ). There exists a matrix $B$ of cluster type $D_{n+1}$ (respectively $A_{2 n-1}$ ) such that the cluster algebra with principal coefficients $\mathcal{A}_{\bullet}(\bar{B})$ is a subquotient of the cluster algebra with principal coefficients $\mathcal{A}_{\bullet}(B)$. In particular any c-vector of $\mathcal{A}_{\bullet}(\bar{B})$ is the folding of some c-vector of $\mathcal{A}_{\bullet}(B)$.

\subsection{Folding of $d$-vectors}

Our next goal is to produce a folding rule for $d$-vectors. From the above example it is natural to fold the vector $d=\left(d_{i}\right)_{i \in I}$ componentwise in this way:

$$
d_{\bar{\imath}}:=\sum_{s \in \bar{\imath}} d_{s}
$$

THE ELECTRONIC JOURNAL OF COMBINATORICS 21(1) (2014), \#P1.3 
Once again the correctness of the above definition is not obvious because, in general, folding is not compatible with the tropicalization map (2.4).

Recall the definition of $D$-matrix given in Section 2.1 .

Lemma 5.10. If $\sigma$ is a stable admissible automorphism of $B$ then the entries in any $D$-matrix of $\mathcal{A}^{\sigma}(B)$ satisfy

$$
d_{\sigma(i) \sigma(j)}=d_{i j} .
$$

Proof. The property holds for the $D$-matrix of the initial cluster. Suppose that $D$ and $D^{\prime}$ correspond to clusters obtained from one another by a single orbit mutation $\mu_{\bar{k}}$ and that the property holds for $D$. The only non trivial case we need to consider is when $j$ is in $\bar{k}$. By (2.6) we have

$$
d_{i j}^{\prime}=-d_{i j}+\max \left(\sum_{t \in I} d_{i t}\left[b_{t k}\right]_{+}, \sum_{t \in I} d_{i t}\left[-b_{t k}\right]_{+}\right)
$$

Using both induction hypotheses and the fact that $\sigma$ is stable admissible we get

$$
d_{i j}^{\prime}=-d_{\sigma(i) \sigma(j)}+\max \left(\sum_{t \in I}-d_{\sigma(i) \sigma(t)}\left[b_{\sigma(t) \sigma(k)}\right]_{+}, \sum_{t \in I}-d_{\sigma(i) \sigma(t)}\left[-b_{\sigma(t) \sigma(k)}\right]_{+}\right)
$$

and we can conclude changing the summation index.

We define the folding $\bar{D}$ of the $D$-matrix $D=\left(d_{i j}\right)_{i j \in I}$ as we $\operatorname{did}$ for $C$-matrices:

$$
d_{\bar{\imath}}:=\sum_{s \in \bar{\imath}} d_{s j}
$$

Thank to the above lemma this definition is independent of the representative $j$.

Proposition 5.11. The matrix $\bar{D}$ satisfies the recursion

$$
d_{\overline{\imath \jmath}}^{\prime}= \begin{cases}-d_{\bar{\imath} \bar{k}}+\max \left(\sum_{\bar{\ell} \in I / \sigma} d_{\bar{\imath} \bar{\ell}}\left[b_{\overline{\ell k}}\right]_{+}, \sum_{\bar{\ell} \in I / \sigma} d_{\bar{\imath} \bar{\ell}}\left[-b_{\overline{\ell k}}\right]_{+},\right) & \bar{\jmath}=\bar{k} \\ d_{\bar{\imath} \bar{\jmath}} & \bar{\jmath} \neq \bar{k} .\end{cases}
$$

if and only if for any $\sigma$-orbit $\bar{\imath}$ the sign of

$$
\sum_{t \in I} d_{s t} b_{t k}
$$

is independent of the representative $s \in \bar{\imath}$. 
Proof. We proceed again by induction. It suffices to show that the property holds for a single mutation. Fix a $\sigma$-orbit $\bar{\imath}$. The only non-trivial case is when $\bar{\jmath}=\bar{k}$. On the one hand we have

$$
d_{\bar{\imath}}^{\prime}=\sum_{s \in \bar{\imath}}\left(-d_{s j}+\max \left(\sum_{t \in I} d_{s t}\left[b_{t k}\right]_{+}, \sum_{t \in I} d_{s t}\left[-b_{t k}\right]_{+}\right)\right) .
$$

On the other hand, for the recursion to be satisfied, we must have

$$
d_{\bar{\imath}}^{\prime}=-\sum_{s \in \bar{\imath}} d_{s j}+\max \left(\sum_{t \in I} \sum_{s \in \bar{\imath}} d_{s t}\left[b_{t k}\right]_{+}, \sum_{t \in I} \sum_{s \in \bar{\imath}} d_{s t}\left[-b_{t k}\right]_{+}\right) .
$$

We need therefore to have

$$
\begin{aligned}
& \sum_{s \in \bar{\imath}}\left(\max \left(\sum_{t \in I} d_{s t}\left[b_{t k}\right]_{+}, \sum_{t \in I} d_{s t}\left[-b_{t k}\right]_{+}\right)\right)= \\
& \max \left(\sum_{s \in \bar{\imath}} \sum_{t \in I} d_{s t}\left[b_{t k}\right]_{+}, \sum_{s \in \bar{\imath}} \sum_{t \in I} d_{s t}\left[-b_{t k}\right]_{+}\right)
\end{aligned}
$$

which holds if and only if the sign of

$$
\sum_{t \in I} d_{s t}\left[b_{t k}\right]_{+}-\sum_{t \in I} d_{s t}\left[-b_{t k}\right]_{+}=\sum_{t \in I} d_{s t} b_{t k}
$$

is independent of the choice of the representative $s \in \bar{\imath}$.

Remark 5.12. Anna Felikson and Pavel Tumarkin found a case of cluster affine type $D$ where the condition of previous proposition does not hold [22]; we thank them for showing us their example.

For our purposes it is enough to show that the condition of Proposition 5.11 holds in the cases of Remark 5.4. Using Lemma 5.10 and the fact that $\sigma$ is stable admissible, it is equivalent to ask the sign of

$$
\sum_{t \in I} d_{i t} b_{t r}
$$

to be independent of the representative $r \in \bar{k}$. We get therefore that the condition is satisfied whenever $k$ is fixed by $\sigma$.

We prefer to work with this third equivalent formulation: the sign of

$$
\sum_{t \in I} d_{i \sigma^{m}(t)} b_{t k}
$$

is independent of $m \in \mathbb{Z}$.

Lemma 5.13. The condition of Proposition 5.11 holds for $B$ of cluster type $D_{n+1}$ endowed with the automorphism $\sigma$ of Remark 5.4. 
Proof. There is only one non-trivial $\sigma$-orbit; in view of previous observations we can assume it is the orbit of $k$. This forces $t \notin \bar{k}$ to be fixed by $\sigma$. Moreover, since $\sigma$ is a stable admissible automorphism $b_{t k}=0$ if $t \in \bar{k}$. Therefore

$$
\sum_{t \in I} d_{i \sigma^{m}(t)} b_{t k}=\sum_{t \in I \backslash \bar{k}} d_{i t} b_{t k}
$$

which is manifestly independent of $m$.

Lemma 5.14. The condition of Proposition 5.11 holds for $B$ of cluster type $A_{2 n-1}$ endowed with the automorphism $\sigma$ of Remark 5.4.

Proof. Note at first that rows of a $D$-matrix associated to a $B$-matrix $B^{\prime}$ in $\mathcal{A}_{0}^{\sigma}(B)$ are again $d$-vectors: they are the $d$-vectors of $\mathcal{A}_{0}^{\sigma}\left(B^{\prime}\right)$ in the $D$-matrix associated to $B$. This follows directly from the surface realization (see Theorem 4.7). In particular, in this case, they are sign-coherent and their support is either a string (if they are positive) or a single vertex (if they are negative).

As before we can assume that $k$ is not fixed by $\sigma$. If the support of the row $i$ does not contain neighbours of both $k$ and $\sigma(k)$ then the statement is clear. We can therefore assume that there is at least one neighbour of each of them in the support of the $i$-th row of $D$.

Let $t_{1}$ and $t_{2}$ be the two neighbours of $k$ and $\sigma(k)$ respectively lying on the shortest path from $k$ to $\sigma(k)$. By the symmetry required for folding $t_{2}=\sigma\left(t_{1}\right)$. Moreover if a row of $D$ contains at least one neighbour of both $k$ and $\sigma(k)$ then it contains both $t_{1}$ and $t_{2}$. We claim that, in this situation,

$$
\sum_{t \in I} d_{i \sigma^{m}(t)} b_{t k}
$$

is either 0 or has the same sign of $b_{t_{1} k}$. Indeed each row of $D$ has at most 2 neighbours of $k$ in its support and the entries of $B$ are either 0 or \pm 1 .

We can therefore conclude our proof: since $\sigma$ is a stable admissible automorphism of $B$ we have:

$$
b_{t_{1} k}=b_{\sigma\left(t_{1}\right) \sigma(k)} .
$$

\subsection{Proof of Theorem 1.1 for types $B_{n}$ and $C_{n}$}

To fix the notation observe that any $B$-matrix of cluster type $B_{n}$ or $C_{n}$ uniquely determines a $\sigma$-invariant matrix of cluster type respectively $D_{n+1}$ or $A_{2 n-1}$ of which it is the folding. We will therefore denote by $\bar{B}$ a matrix of cluster type $B_{n}$ or $C_{n}$ and by $B$ its unfolding.

Let $\pi(\mathcal{V}(B))$ be the image of the set $\mathcal{V}(B)$ under the folding map

$$
\pi: \begin{aligned}
& \mathcal{V}(B) \longrightarrow \mathbb{Z}^{\bar{I}} \\
&\left(v_{i}\right)_{i \in I} \longmapsto \\
&\left(\sum_{s \in \bar{\imath}} v_{s}\right)_{\bar{\imath} \in \bar{I}}
\end{aligned}
$$

and recall the definition of the sets $\mathcal{W}\left(B_{n}\right)$ and $\mathcal{W}\left(C_{n}\right)$ from Section 3. 
Proposition 5.15. For any matrix $\bar{B}$ of cluster type $B_{n}$ or $C_{n}$ we have

$$
\mathcal{V}(\bar{B})=\pi(\mathcal{V}(B)) .
$$

Proof. The claim is clear once we observe that the diagrams in $\mathcal{W}\left(B_{n}\right)$ and $\mathcal{W}\left(C_{n}\right)$ are obtained precisely by folding diagrams from $\mathcal{W}\left(D_{n+1}\right)$ and $\mathcal{W}\left(A_{2 n-1}\right)$ embedded in $X(B)$.

We have now the tools we need to deduce Theorem 1.1 for types $B_{n}$ and $C_{n}$ from the same result for types $A_{n}$ and $D_{n}$.

Proposition 5.16. For any matrix $\bar{B}$ of cluster type $B_{n}$ or $C_{n}$ we have

$$
\mathcal{C}_{+}(\bar{B}) \subset \mathcal{V}(\bar{B})
$$

Proof. Combining Corollary 5.9, Proposition 4.10 and Proposition 5.15 we have

$$
\mathcal{C}_{+}(\bar{B}) \subset \pi\left(\mathcal{C}_{+}(B)\right)=\pi(\mathcal{V}(B))=\mathcal{V}(\bar{B}) .
$$

Proposition 5.17. For any matrix $\bar{B}$ of cluster type $B_{n}$ or $C_{n}$ we have

$$
\mathcal{D}(\bar{B}) \subset \mathcal{V}(\bar{B})
$$

Proof. Combining Lemmata 5.13 and 5.14 with Proposition 4.13, Proposition 4.10 and Proposition 5.15 we have

$$
\mathcal{D}(\bar{B}) \subset \pi(\mathcal{D}(B))=\pi(\mathcal{V}(B))=\mathcal{V}(\bar{B}) .
$$

To conclude we need one last lemma.

Lemma 5.18. For any matrix $\bar{B}$ of cluster type $B_{n}$ or $C_{n}$ we have

$$
\mathcal{C}_{+}^{b}(\bar{B})=\pi\left(\mathcal{C}_{+}^{b}(B)\right)
$$

and

$$
\mathcal{D}^{b}(\bar{B})=\pi\left(\mathcal{D}^{b}(B)\right) .
$$

Proof. The claim follows directly from the following observation: a matrix $\bar{B}$ is bipartite if and only if its unfolding $B$ is bipartite. We get equalities (as opposed to inclusions) because any two bipartite matrices of cluster type $D_{n+1}$ or $A_{2 n-1}$ are connected by orbit mutations.

Proposition 5.19. For any matrix $\bar{B}$ of cluster type $B_{n}$ or $C_{n}$ we have

$$
\mathcal{V}(\bar{B}) \subset \mathcal{C}_{+}(\bar{B})
$$

and

$$
\mathcal{V}(\bar{B}) \subset \mathcal{D}(\bar{B})
$$


Proof. We show only the second condition; the first one is obtained in the same way. Using Proposition 5.15, Proposition 4.14, equation (4.3), and Lemma 5.18 we get

$$
\mathcal{V}(\bar{B})=\pi(\mathcal{V}(B)) \subset \pi(\mathcal{D}(B))=\pi\left(\mathcal{D}^{b}(B)\right)=\mathcal{D}^{b}(\bar{B}) \subset \mathcal{D}(\bar{B})
$$

For completeness we record also the following equalities (of which Theorem 1.4 is a direct consequence).

Corollary 5.20. For any matrix $\bar{B}$ of cluster type $B_{n}$ or $C_{n}$ we have

$$
\begin{aligned}
\mathcal{C}_{+}^{b}(\bar{B}) & =\mathcal{C}_{+}(\bar{B}), \\
\mathcal{D}^{b}(\bar{B}) & =\mathcal{D}(\bar{B}), \\
\mathcal{C}_{+}^{b}(\bar{B}) & =\mathcal{D}^{b}(\bar{B}) .
\end{aligned}
$$

\section{Proof of Theorem 1.3}

Here we derive Theorem 1.3. The claim (4) is a direct consequence of our description of $c$ - and $d$-vectors in Theorem 1.1. For simply-laced types claims (1) and (3) follow from Corollaries 2.8 and 2.11. However, for types $A_{n}$ and $D_{n}$, we provide a direct proof using Theorem 1.1 without referring to the representation-theoretic results of Section 2.

As we did before we deal with types $A_{n}$ and $D_{n}$ first; we will use again a folding argument to deduce the results for types $B_{n}$ and $C_{n}$.

\subsection{Types $A_{n}$ and $D_{n}$}

Let $B$ be any skew-symmetric integer matrix of cluster type either $A_{n}$ or $D_{n}$. Having built an explicit list of all the positive $c$-vectors and non-initial $d$-vectors for the cluster algebra $\mathcal{A}_{\bullet}(B)$ with principal coefficients we can give a combinatorial proof of Theorem 1.3 .

Proposition 6.1. All c-vectors and d-vectors of $\mathcal{A}_{\bullet}(B)$ are roots in the root system associated to the Cartan counterpart of $B$. Each of them is real if and only if its support in $X(B)$ is a tree.

Proof. It suffices to establish the claim for positive $c$-vectors. We are dealing with a local property: since the support of any $c$-vector $c$ of $\mathcal{A}_{\bullet}(B)$ is a connected sub-diagram of $X(B)$ it suffices to show that $c$ is a root in the root system associated to its support.

The claim is clear for type $A_{n}$ and for cases I, II and III of type $D_{n}$ : they are all roots in the corresponding finite type root system.

Applying in sequence the simple reflections corresponding to the outermost node with multiplicity 2 we can reduce case VII to case VI. We can then "trim the branches" reflecting each time with respect to a leaf of the diagram. After these reductions we are 
(a)

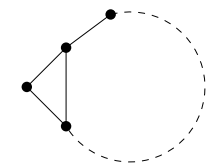

(b)

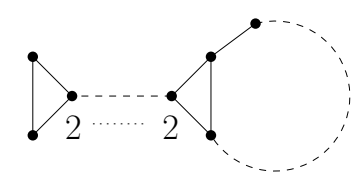

(c)

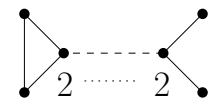

(d)

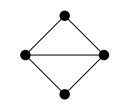

Figure 6.1: Reduced $c$-vectors.

left with the four cases in Figure 6.1. They all correspond to imaginary roots. Indeed let $c$ be any of these reduced $c$-vectors and let $A$ be the generalized Cartan matrix associated to its support, then all the components of the vector $A c$ are non-positive which is exactly the condition of [34, Lemma 5.3].

Let $\langle\cdot, \cdot\rangle$ be the Euler form of the quiver $Q=Q(B)$ associated to $B$; it is defined on roots as follows:

$$
\left\langle\sum_{i \in I} c_{i} \alpha_{i}, \sum_{i \in I} d_{i} \alpha_{i}\right\rangle:=\sum_{i \in I} c_{i} d_{i}-\sum_{b_{i j}>0} b_{i j} c_{j} d_{i} .
$$

To show that elements of $\mathcal{C}_{+}(B)=\mathcal{D}(B)$ are Schur roots we will use the following result of A. Schofield ([48, Theorem 6.2]).

Theorem 6.2. Let $\alpha$ be a positive root that is not a Schur root then $\alpha$ satisfies one of the following conditions:

1. $\langle\alpha, \alpha\rangle=0$ and there are a positive (imaginary) root $\beta$ and a positive integer $k$ such that $\alpha=k \beta$.

2. $\alpha$ is the sum of two positive roots, one of them (call it $\beta$ ) is real and satisfies

$$
\langle\alpha, \beta\rangle>0 \quad \text { and } \quad\langle\beta, \alpha\rangle>0 .
$$

Let $A=A(B)$ be the Cartan counterpart of $B$. As noted in [48], if $\alpha$ is an imaginary root that is not Schur, there are few possibilities for the positive real root $\beta$ satisfying (2). Namely, if $w$ is the element of the Weyl group such that all the components of the vector $A w(\alpha)$ are non positive then $w(\beta)$ has to be a negative real root.

Proposition 6.3. All the vectors in $\mathcal{C}_{+}(B)=\mathcal{D}(B)$ are Schur roots of $\Delta(A(B))$.

Proof. We are dealing still with a local property so we can assume that the $c$-vector we consider has full support.

It is well known that if $X(B)$ is a finite type Dynkin diagram then any root is a Schur root (every indecomposable $k Q(B)$-module is rigid if $Q(B)$ is an orientation of a Dynkin 
diagram); therefore we need only to concentrate on cases IV, V, VI, VII, and VIII of type $D_{n}$. Let $c$ be any of these $c$-vectors, they are all imaginary roots. None of them is an integer multiple of a root so case (1) of Theorem 6.2 is excluded and we need to show only that we are not in case (2).

As noted in Proposition 6.1 the elements $w$ of the Weyl group we need to apply to $c$ are those "trimming the branches"; since the roots $\beta$ we are looking for change sign when acted on by $w$ their support must be contained in only one of those appendices; we can therefore assume that there is only one appendix in the weighted diagram of $c$. Label the nodes on such an appendix with $\{1 \ldots, n-1\}$ starting from the leaf; let $n$ be the node the appendix is connected to and let $m$ be the innermost node with multiplicity 1 in the appendix. It is clear that the element $w$ we are looking for is then $s_{n-1} \ldots s_{1}$ in cases IV, V, VI, and VIII and $s_{n-1} \ldots s_{1} s_{n} \ldots s_{m+1}$ in case VII. The possible roots $\beta$ are then

$$
\alpha_{1}+\cdots+\alpha_{k}
$$

for $k \in\{1, \ldots, n-1\}$ in cases IV, V, VI, and VIII and

$$
\begin{array}{rc}
\alpha_{1}+\cdots+\alpha_{k+1} & m \leqslant k \leqslant n-1 \\
\alpha_{1}+\cdots+\alpha_{k} & k<m \\
\alpha_{m+1}+\cdots+\alpha_{k} & m+1 \leqslant k \leqslant n
\end{array}
$$

in case VII. By direct inspection we get that in all cases, regardless of the orientations, one of the two integers $\langle c, \beta\rangle$ and $\langle\beta, c\rangle$ is non-positive.

Proposition 6.4. The cardinality $\left|\mathcal{C}_{+}(B)\right|=|\mathcal{D}(B)|$ depends only on the cluster type of $B$; it is equal to $n(n+1) / 2$ if $B$ is of cluster type $A_{n}$ and $n(n-1)$ if $B$ is of cluster type $D_{n}$.

Proof. Fix an element $X(B)$ of $\mathcal{X}(B)$. We need to count in how many different ways any diagrams from $\mathcal{W}(B)$ can be embedded in $X(B)$.

This count, for type $A_{n}$, was done by Parson ([44, Lemma 5.8]) by noting that any embedding of a string is determined by the positions of its endpoints.

Let us consider type $D_{n}$; there are four cases to be considered depending on which of the four diagrams in Figure 3.6 describes $X(B)$. We present case $(\mathrm{d})$ : it involves all the techniques and it is the most complex one. The other cases can be dealt with in a similar fashion.

The only weighted diagrams that can be embedded in a Dynkin diagram shaped as (d) are I, VI, VII, and VIII from Figure 3.7. An embedding of any of those is uniquely determined by a pair of vertices in $X(B)$; for I (with at least two nodes), and VIII they are the two leaves; for VII they are the only leaf and the leftmost node with weight 2. For VI and strings of length 1 the two vertices of $X(B)$ coincide.

We are going to reverse this observation to count embeddings. Suppose that the central cycle contains $k$ vertices.

To each pair of vertices $i$ and $j$ not in the central cycle we can associate precisely two embeddings: if they belong to different components (say $X^{\prime}$ and $X^{\prime \prime}$ ) we have two 
strings passing on either side of the central cycle. If $i$ and $j$ belong to the same type- $A_{m}$ component (say $X^{\prime}$ ) and are distinct then we have a string connecting $i$ to $j$ completely contained in $X^{\prime}$ and a weighted diagram of type VII or VIII depending on the relative position of $i$ and $j$. Finally if $i=j$ then we have a single point and a weighted diagram of type VI. They sum up to $(n-k)(n-k+1)$ embeddings.

If one of the two vertices, say $i$, is in the central cycle and $j$ is in the component $X^{\prime}$ then there are two possibilities: if $i$ is one of the two vertices adjacent to $X^{\prime}$ then there is only one embedding associated to the pair $i$ and $j$ : the shortest string connecting them. Otherwise there are two strings that we can embed into $X(B)$ depending on the side of the central cycle we cross. Therefore there are $2(k-2)(n-k)+2(n-k)$ embeddings with one vertex in a type- $A_{m}$ component and a vertex in the central cycle.

Finally if both $i$ and $j$ are in the central cycle we need to distinguish three cases: they can coincide (yielding embedding of single nodes), they can be adjacent (and produce embedding of strings of length 2). Otherwise they produce precisely two embedding of strings. In total there are $k^{2}-k$ embeddings induced by pair of vertices in the central cycle.

Summing up all the contributions we get

$$
(n-k)(n-k+1)+2(k-2)(n-k)+2(n-k)+k^{2}-k=n^{2}-n
$$

as desired.

\subsection{Types $B_{n}$ and $C_{n}$}

To extend the above results to types $B_{n}$ and $C_{n}$ we will use the following general fact on the folding of root systems.

Proposition 6.5. Let $B$ be a skew-symmetrizable integer matrix together with an admissible automorphism $\sigma$ and denote by $A=A(B)$ its Cartan counterpart. Let $\bar{B}$ be the image of $B$ under the folding map $\pi$ and $\bar{A}=A(\bar{B})$ the Cartan counterpart of $\bar{B}$. Let $\left\{\alpha_{i}\right\}_{i \in I}$ be the simple roots for $\Delta(A)$ and $\left\{\alpha_{\bar{\imath}}\right\}_{\bar{\imath} \in I / \sigma}$ be the simple roots for $\Delta(\bar{A})$.

Define the linear map $\pi$ from the root lattice of $\Delta(A)$ to the root lattice of $\Delta(\bar{A})$ by

$$
\pi\left(\alpha_{i}\right):=\alpha_{\bar{\imath}} .
$$

Then for any $\alpha \in \Delta(A)$ we have $\pi(\alpha) \in \Delta(\bar{A})$.

Proof. This argument is a refinement of [52, Proposition A.7]; there the result is stated only for finite type root systems.

Observe first that the map $\pi$ commutes with "orbit reflections"

$$
s_{\bar{\imath}}^{\sigma}:=\prod_{t \in \bar{\imath}} s_{t}
$$

that is for any root $\alpha$

$$
s_{\bar{\imath}}(\pi(\alpha))=\pi\left(s_{\bar{\imath}}^{\sigma}(\alpha)\right)
$$


Orbit reflections are well defined because, by admissibility of $\sigma$, we have

$$
a_{i_{1} i_{2}}=0
$$

for any pair $i_{1} \neq i_{2}$ in the same $\sigma$-orbit $\bar{\imath}$. It is sufficient to verify (6.1) on simple roots; we have

$$
s_{\bar{\imath}}\left(\pi\left(\alpha_{j}\right)\right)=s_{\bar{\imath}}\left(\alpha_{\bar{\jmath}}\right)=\alpha_{\bar{\jmath}}-a_{\bar{\imath} \bar{\jmath}} \alpha_{\bar{\imath}}=\pi\left(\alpha_{j}-\sum_{t \in \bar{\imath}} a_{t j} \alpha_{t}\right)=\pi\left(s_{\bar{\imath}}^{\sigma}\left(\alpha_{j}\right)\right) .
$$

Back to our problem, without loss of generality we can assume $\alpha$ to be a positive root; we will proceed by induction on

$$
\operatorname{ht}(\alpha)=\operatorname{ht}\left(\sum_{i \in I} c_{i} \alpha_{i}\right):=\sum_{i \in I} c_{i}
$$

If $\operatorname{ht}(\alpha)=1$ then $\alpha=\alpha_{i}$ for some $i \in I$; thus $\pi(\alpha)=\alpha_{\bar{\imath}}$. Suppose now that $\operatorname{ht}(\alpha)>1$. If all the components of the vector $\bar{A} \pi(\alpha)$ are negative then $\pi(\alpha)$ is an imaginary root (see [34, Lemma 5.3]). Otherwise let $\bar{\imath}$ be such that

$$
(\bar{A} \pi(\alpha))_{\bar{\imath}}>0
$$

Set $\alpha^{\prime}:=s_{\bar{\imath}}^{\sigma}(\alpha)$. Since $\bar{\imath}$ is disconnected, in view of (6.2) $\alpha^{\prime}$ is a positive root and

$$
\text { ht }\left(\alpha^{\prime}\right)<\operatorname{ht}(\alpha) \text {. }
$$

By induction hypothesis then $\pi\left(\alpha^{\prime}\right)$ is a positive root in the root system of $\Delta(\bar{A})$ therefore so is

$$
\pi(\alpha)=s_{\bar{\imath}}\left(s_{\bar{\imath}}(\pi(\alpha))\right)=s_{\bar{\imath}}\left(\pi\left(s_{\bar{\imath}}^{\sigma}(\alpha)\right)\right)=s_{\bar{\imath}}\left(\pi\left(\alpha^{\prime}\right)\right) .
$$

Note that the folding of roots agrees with the folding of both $c-$ and $d$-vectors.

Proposition 6.6. Let $B$ be a skew-symmetrizable integer matrix of cluster type $B_{n}$ or $C_{n}$. All the c-vectors and d-vectors of $\mathcal{A}_{\mathbf{\bullet}}(B)$ are roots in the root system $\Delta(A(B))$.

Proof. It is enough to consider positive $c$-vectors. By Corollary 5.9 any element of $\mathcal{C}_{+}(B)$ is the image of some $c$-vector of a cluster algebra of type $D_{n+1}$ or $A_{2 n-1}$. By Proposition 6.1 the latter are roots in the root system associated to the unfolding of $B$. Our claim follows then directly from Proposition 6.5.

Proposition 6.7. Any c-vector (d-vector) of $\mathcal{A}_{\bullet}(B)$ of type $B_{n}$ or $C_{n}$ is a real root if and only if its support is a tree.

Proof. If the support of the vector we are considering is a tree there is nothing to show. In all other cases we can "trim the branches" and check directly as we did in Proposition 6.1 .

Proposition 6.8. For any B-matrix of cluster type either $B_{n}$ or $C_{n}$ the cardinality of $\mathcal{V}(B)$ is equal to $n^{2}$.

Proof. This claim does not follow directly from folding. Nevertheless it is straightforward to apply the same argument of Proposition 6.4 to perform the counting. 


\section{Acknowledgments}

We thank Anna Felikson, Bernhard Keller, Robert Marsh, Gregg Musiker, Satoshi Naito, Idun Reiten, Pavel Tumarkin, Toshiyuki Tanisaki, Gordana Todorov, and Jerzy Weyman for useful discussion. We thank Alfredo Nájera Chávez for letting us use his result [40] prior to the publication, Hugh Thomas for his guidance on representation theory, and the anonymous referee for the useful suggestions. We also thank MSRI, Berkeley for financial support, for the computer facilities used when dealing with exceptional types, and for providing the ideal environment where the last stage of this work was done.

We would like to dedicate this paper to the memory of Andrei Zelevinsky. We are grateful to him for sharing his insights and ideas on the subject, and suggesting the coincidence of the $c$ - and $d$-vectors to us at the early stage of this work before the appearance of the paper [42]. The second author is especially grateful to him for his guidance, support and constant spur during the course of his Ph.D. studies.

\section{References}

[1] Ibrahim Assem, Daniel Simson, and Andrzej Skowroński. Elements of the representation theory of associative algebras. Vol. 1, volume 65 of London Mathematical Society Student Texts. Cambridge University Press, Cambridge, 2006. Techniques of representation theory.

[2] Michael Barot, Christof Geiss, and Andrei Zelevinsky. Cluster algebras of finite type and positive symmetrizable matrices. J. London Math. Soc. (2), 73(3):545-564, 2006.

[3] Nicolas Bourbaki. Lie groups and Lie algebras. Chapters 4-6. Elements of Mathematics (Berlin). Springer-Verlag, Berlin, 2002. Translated from the 1968 French original by Andrew Pressley.

[4] Aslak Bakke Buan, Robert Marsh, Markus Reineke, Idun Reiten, and Gordana Todorov. Tilting theory and cluster combinatorics. Adv. Math., 204(2):572-618, 2006.

[5] Aslak Bakke Buan and Robert J. Marsh. Denominators in cluster algebras of affine type. J. Algebra, 323(8):2083-2102, 2010.

[6] Aslak Bakke Buan, Robert J. Marsh, and Idun Reiten. Cluster-tilted algebras of finite representation type. J. Algebra, 306(2):412-431, 2006.

[7] Aslak Bakke Buan, Robert J. Marsh, and Idun Reiten. Cluster-tilted algebras. Trans. Amer. Math. Soc., 359(1):323-332 (electronic), 2007.

[8] Aslak Bakke Buan, Robert J. Marsh, and Idun Reiten. Cluster mutation via quiver representations. Comment. Math. Helv., 83(1):143-177, 2008.

[9] Aslak Bakke Buan, Robert J. Marsh, and Idun Reiten. Denominators of cluster variables. J. Lond. Math. Soc. (2), 79(3):589-611, 2009.

[10] Aslak Bakke Buan, Robert J. Marsh, Idun Reiten, and Gordana Todorov. Clusters and seeds in acyclic cluster algebras. Proc. Amer. Math. Soc., 135(10):3049-3060 
(electronic), 2007. With an appendix coauthored in addition by P. Caldero and B. Keller.

[11] Aslak Bakke Buan and Dagfinn F. Vatne. Derived equivalence classification for cluster-tilted algebras of type $A_{n}$. J. Algebra, 319(7):2723-2738, 2008.

[12] P. Caldero, F. Chapoton, and R. Schiffler. Quivers with relations arising from clusters ( $A_{n}$ case). Trans. Amer. Math. Soc., 358(3):1347-1364, 2006.

[13] Philippe Caldero, Frédéric Chapoton, and Ralf Schiffler. Quivers with relations and cluster tilted algebras. Algebr. Represent. Theory, 9(4):359-376, 2006.

[14] Philippe Caldero and Bernhard Keller. From triangulated categories to cluster algebras. II. Ann. Sci. École Norm. Sup. (4), 39(6):983-1009, 2006.

[15] Giovanni Cerulli Irelli. Cluster Algebras of Type $A_{\mathbf{2}}^{(\mathbf{1})}$. Algebr. Represent. Theory, 15(5):977-1021, 2012.

[16] L. Demonet. Mutations of group species with potentials and their representations. Applications to cluster algebras. ArXiv e-prints, March 2010.

[17] Laurent Demonet. Categorification of skew-symmetrizable cluster algebras. Algebr. Represent. Theory, 14(6):1087-1162, 2011.

[18] Bangming Deng, Jie Du, Brian Parshall, and Jianpan Wang. Finite dimensional algebras and quantum groups, volume 150 of Mathematical Surveys and Monographs. American Mathematical Society, Providence, RI, 2008.

[19] Harm Derksen, Jerzy Weyman, and Andrei Zelevinsky. Quivers with potentials and their representations II: applications to cluster algebras. J. Amer. Math. Soc., 23(3):749-790, 2010.

[20] Vlastimil Dlab and Claus Michael Ringel. Indecomposable representations of graphs and algebras. Mem. Amer. Math. Soc., 6(173):v+57, 1976.

[21] G. Dupont. An approach to non-simply laced cluster algebras. J. Algebra, 320(4):1626-1661, 2008.

[22] Anna Felikson and Pavel Tumarkin. Private communication.

[23] Vladimir V. Fock and Alexander B. Goncharov. Dual Teichmüller and lamination spaces. In Handbook of Teichmüller theory. Vol. I, volume 11 of IRMA Lect. Math. Theor. Phys., pages 647-684. Eur. Math. Soc., Zürich, 2007.

[24] Sergey Fomin, Michael Shapiro, and Dylan Thurston. Cluster algebras and triangulated surfaces. I. Cluster complexes. Acta Math., 201(1):83-146, 2008.

[25] Sergey Fomin and Dylan Thurston. Cluster algebras and triangulated surfaces. II. lambda lengths. preprint, 2008.

[26] Sergey Fomin and Andrei Zelevinsky. Cluster algebras. I. Foundations. J. Amer. Math. Soc., 15(2):497-529 (electronic), 2002.

[27] Sergey Fomin and Andrei Zelevinsky. Cluster algebras. II. Finite type classification. Invent. Math., 154(1):63-121, 2003. 
[28] Sergey Fomin and Andrei Zelevinsky. Cluster algebras. IV. Coefficients. Compos. Math., 143(1):112-164, 2007.

[29] Michael Gekhtman, Michael Shapiro, and Alek Vainshtein. Cluster algebras and Weil-Petersson forms. Duke Math. J., 127(2):291-311, 2005.

[30] Thilo Henrich. Mutation classes of diagrams via infinite graphs. Math. Nachr., 284(17-18):2184-2205, 2011.

[31] Andrew Hubery. Representations of a quiver with automorphism: generalising a theorem of Kac. In Representations of algebras and related topics, volume 45 of Fields Inst. Commun., pages 187-200. Amer. Math. Soc., Providence, RI, 2005.

[32] V. G. Kac. Infinite root systems, representations of graphs and invariant theory. Invent. Math., 56(1):57-92, 1980.

[33] V. G. Kac. Infinite root systems, representations of graphs and invariant theory. II. J. Algebra, 78(1):141-162, 1982.

[34] Victor G. Kac. Infinite-dimensional Lie algebras. Cambridge University Press, Cambridge, third edition, 1990.

[35] Bernhard Keller. Quiver mutation in Java. http://people.math.jussieu.fr/ keller/quivermutation/.

[36] Robert Marsh and Idun Reiten. Private communication.

[37] Gregg Musiker, Ralf Schiffler, and Lauren Williams. Positivity for cluster algebras from surfaces. Adv. Math., 227(6):2241-2308, 2011.

[38] Gregg Musiker and Christian Stump. A compendium on the cluster algebra and quiver package in Sage. Sém. Lothar. Combin., 65:Art. B65d, 67, 2010/12.

[39] K. Nagao. Donaldson-Thomas theory and cluster algebras. ArXiv e-prints, February 2010.

[40] A. Nájera Chávez. to appear.

[41] A. Nájera Chávez. On the c-vectors and g-vectors of the Markov cluster algebra. ArXiv e-prints, December 2011.

[42] A. Nájera Chávez. On the c-vectors of an acyclic cluster algebra. ArXiv e-prints, March 2012.

[43] Tomoki Nakanishi and Andrei Zelevinsky. On tropical dualities in cluster algebras. In Algebraic groups and quantum groups, volume 565 of Contemp. Math., pages 217-226. Amer. Math. Soc., Providence, RI, 2012.

[44] M. J. Parsons. Companion bases for cluster-tilted algebras. ArXiv e-prints, November 2011.

[45] Pierre-Guy Plamondon. Cluster algebras via cluster categories with infinitedimensional morphism spaces. Compos. Math., 147(6):1921-1934, 2011.

[46] N. Reading and D. E Speyer. Combinatorial frameworks for cluster algebras. ArXiv e-prints, November 2011. 
[47] Claus Michael Ringel. Cluster-concealed algebras. Adv. Math., 226(2):1513-1537, 2011.

[48] Aidan Schofield. General representations of quivers. Proc. London Math. Soc. (3), 65(1):46-64, 1992.

[49] D. Speyer and H. Thomas. Acyclic cluster algebras revisited. ArXiv e-prints, March 2012.

[50] W. A. Stein et al. Sage Mathematics Software (Version 5.3). The Sage Development Team, 2012. http://www . sagemath.org.

[51] Christian Stump. To appear.

[52] Toshiyuki Tanisaki. Lie algebras and quantum groups. Kyoritsu-Shuppan, 2002. in Japanese.

[53] Thao Tran. F-polynomials in quantum cluster algebras. Algebr. Represent. Theory, 14(6):1025-1061, 2011.

[54] Dagfinn F. Vatne. The mutation class of $D_{n}$ quivers. Comm. Algebra, 38(3):11371146, 2010.

[55] Shih-Wei Yang and Andrei Zelevinsky. Cluster algebras of finite type via Coxeter elements and principal minors. Transform. Groups, 13(3-4):855-895, 2008.

\section{A Type $D_{n}$ analysis}

We provide here the detailed case analysis required to prove both Propositions 4.11 and 4.10 in type $D_{n}$ at the same time.

As explained above, any multilamination corresponding to an initial triangulation decomposes the surface $S$ into pieces (see Figure 4.6 for an example). Any quadrilateral can intersect positively at most laminations contained in three different pieces. We need therefore to consider, for any quadrilateral in Figure 4.3, all the possible ways of inscribing it in a surface with at most three pieces. These configurations are listed in the leftmost column of the following tables.

For each of them we distinguish five sub-cases (the other five columns) depending on how the multilamination looks around the puncture (cf. Figure 4.5).

For any given configuration and choice of lamination around the puncture we provide a bipartite quadrilateral giving rise to the same $c$-vector as the original triangulation and we record which template $c$-vector from Figure 3.7 we get. 


\begin{tabular}{ll|l|l|l|l|}
$\begin{array}{c}\text { Configuration on } \\
\text { the surface }\end{array}$ & $\begin{array}{c}\text { Plain } n \text {-gon } \\
(n \geq 3)\end{array}$ & Fotched $n$-gon \\
$(n \geq 3)$
\end{tabular}




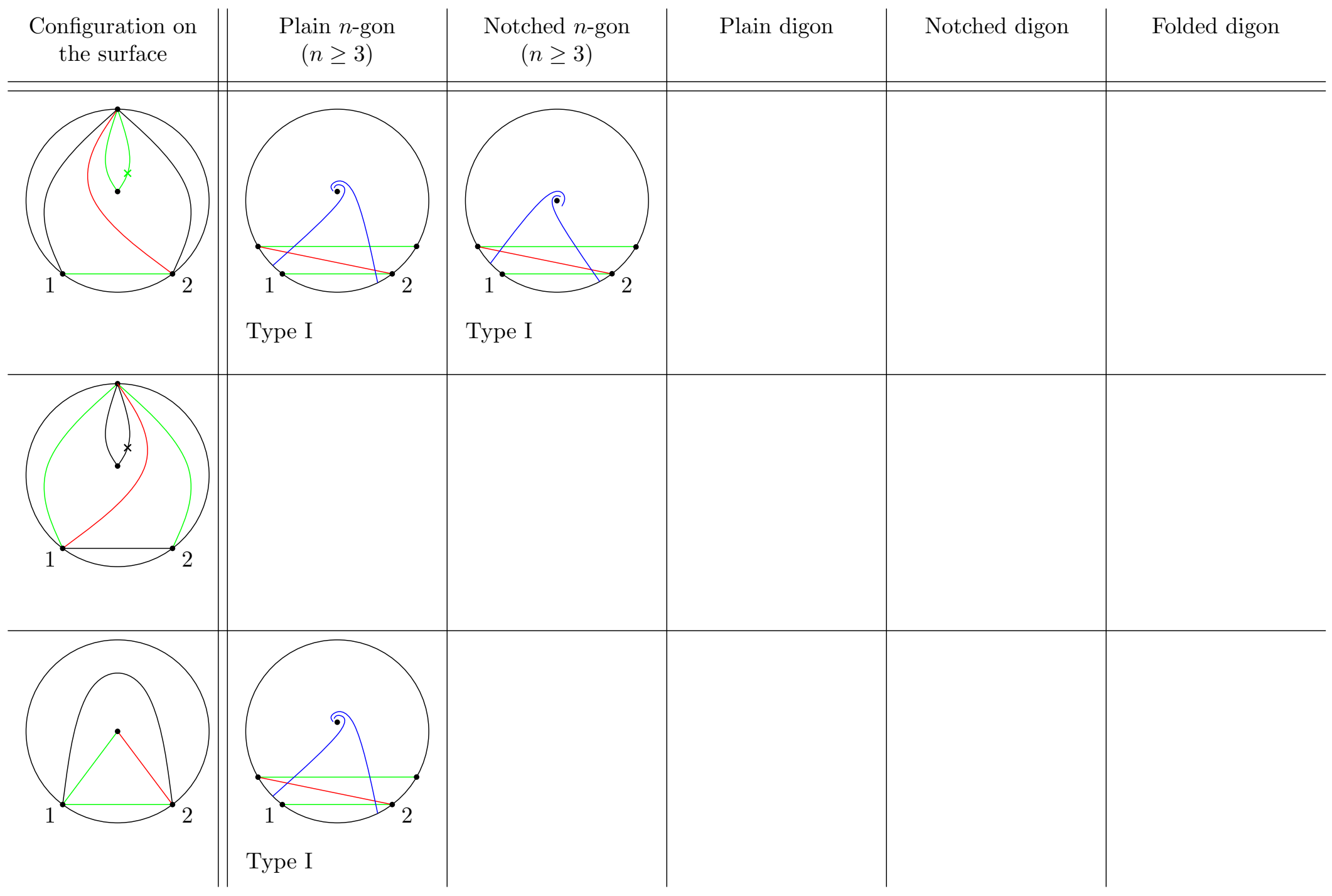




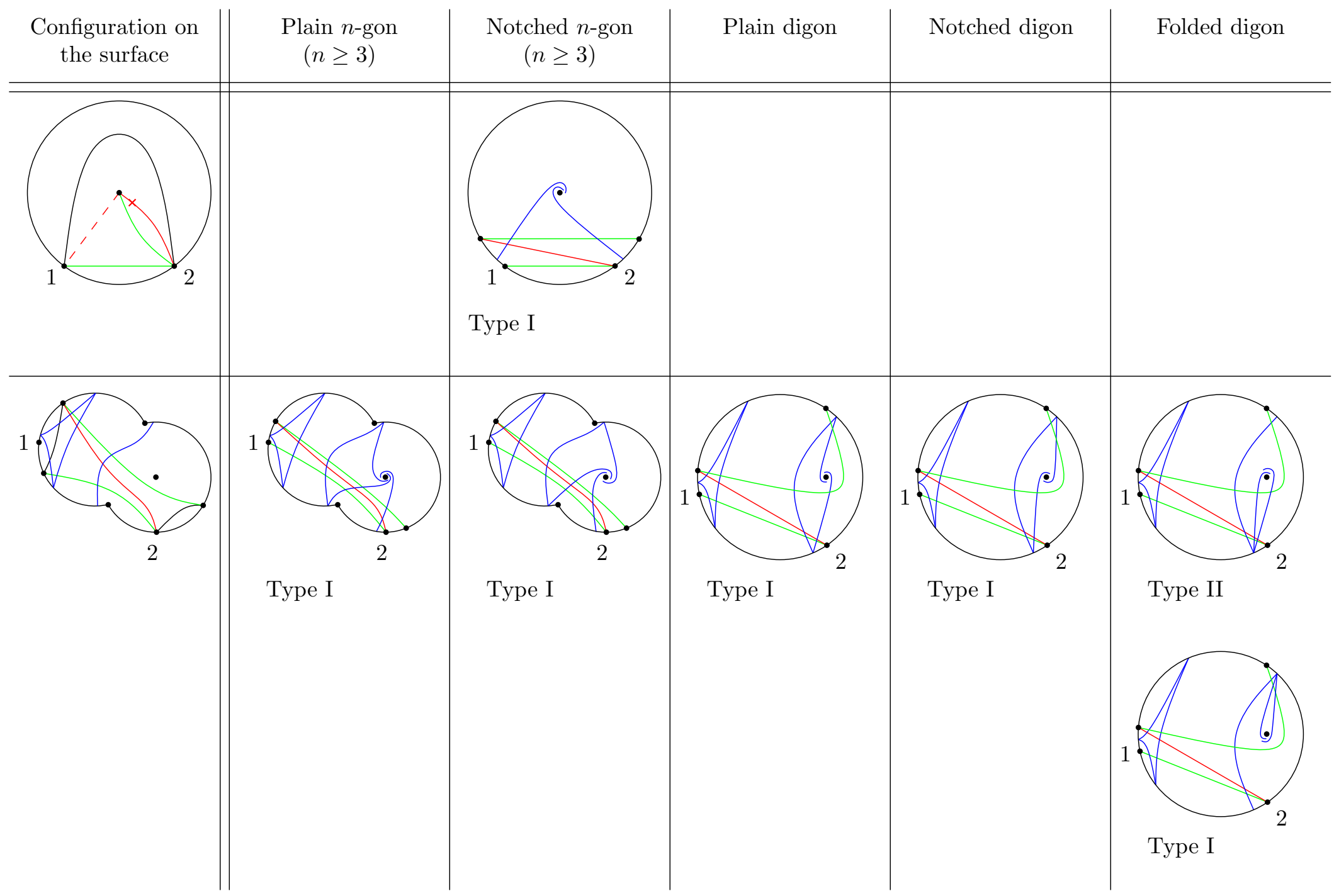




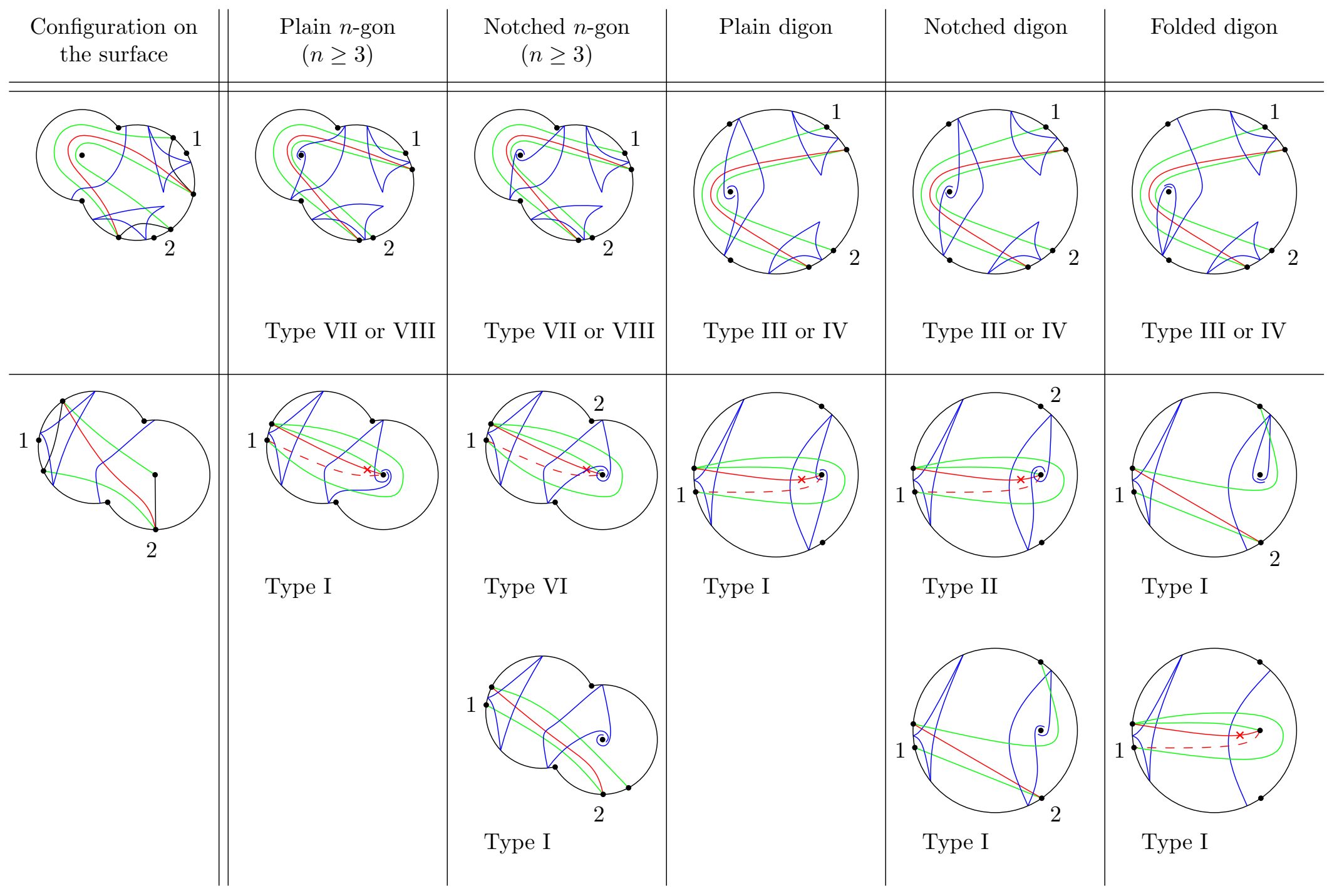




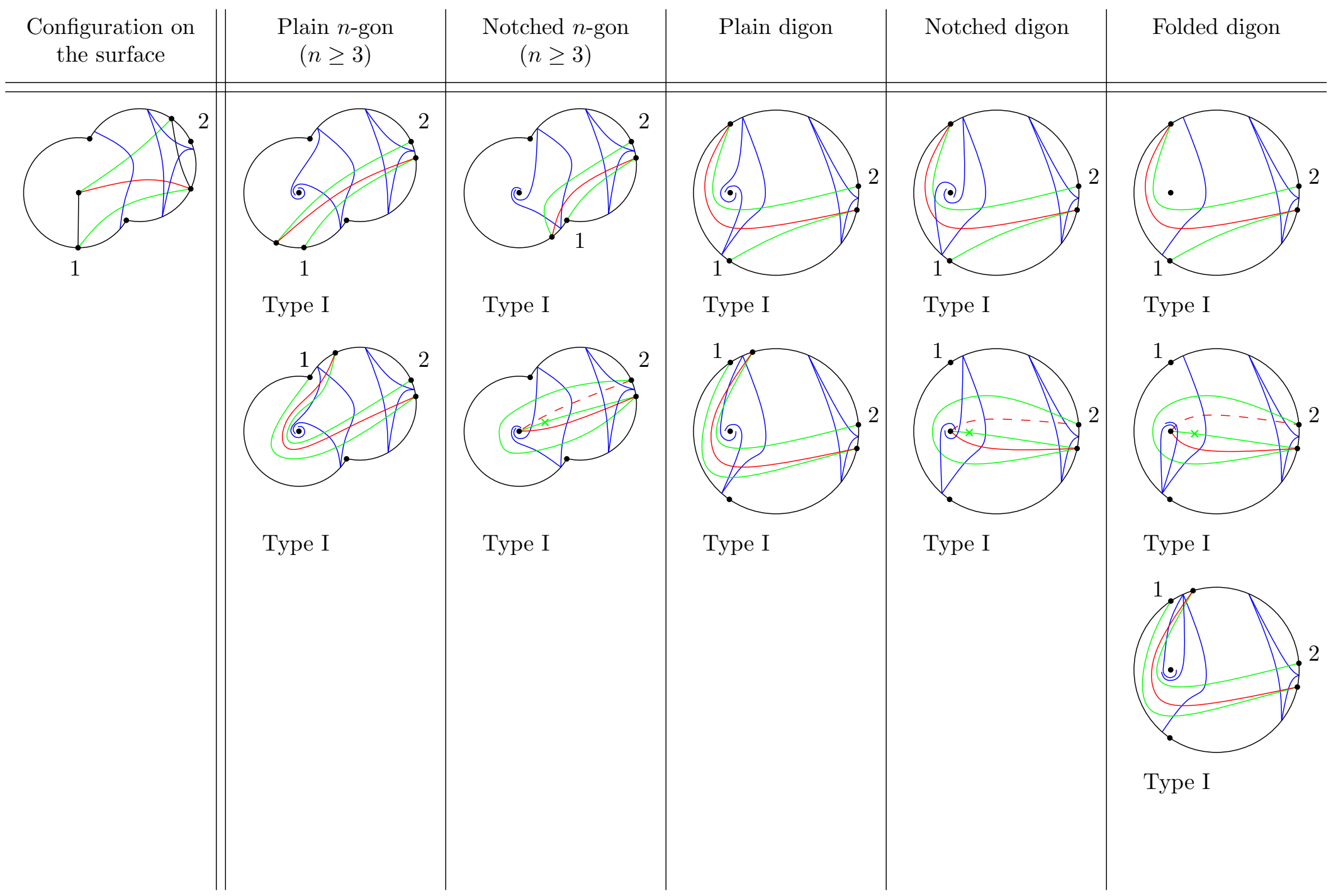




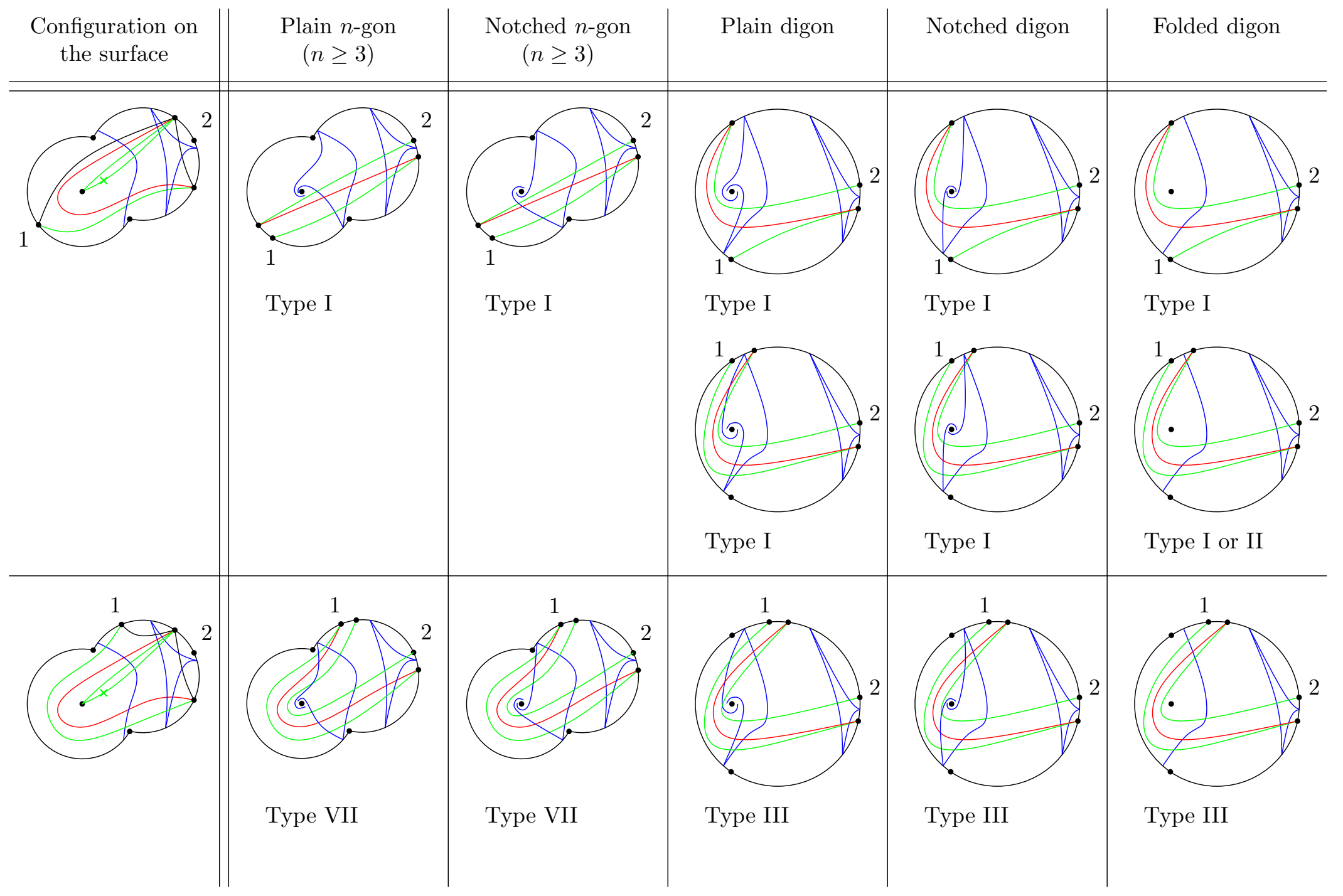




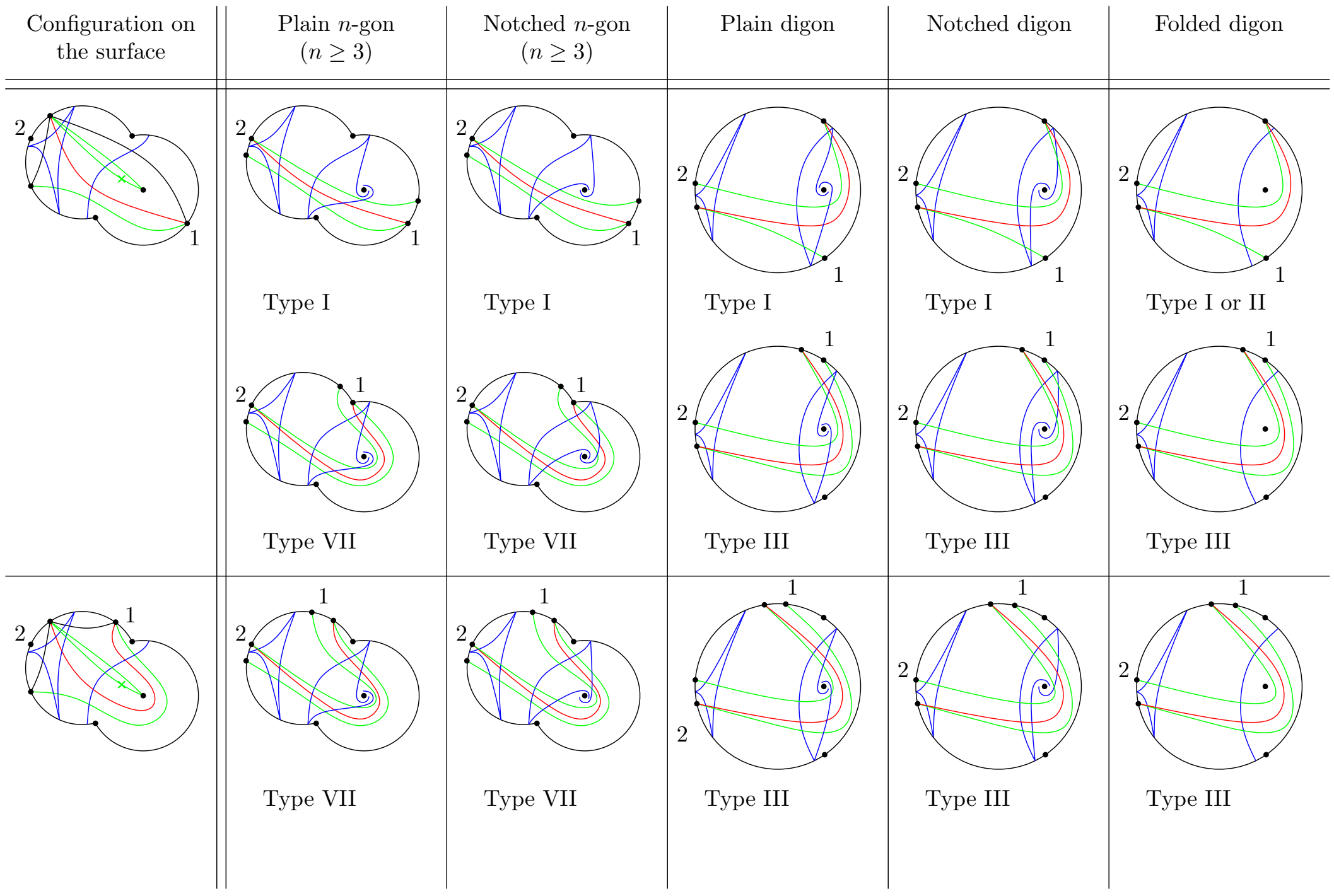




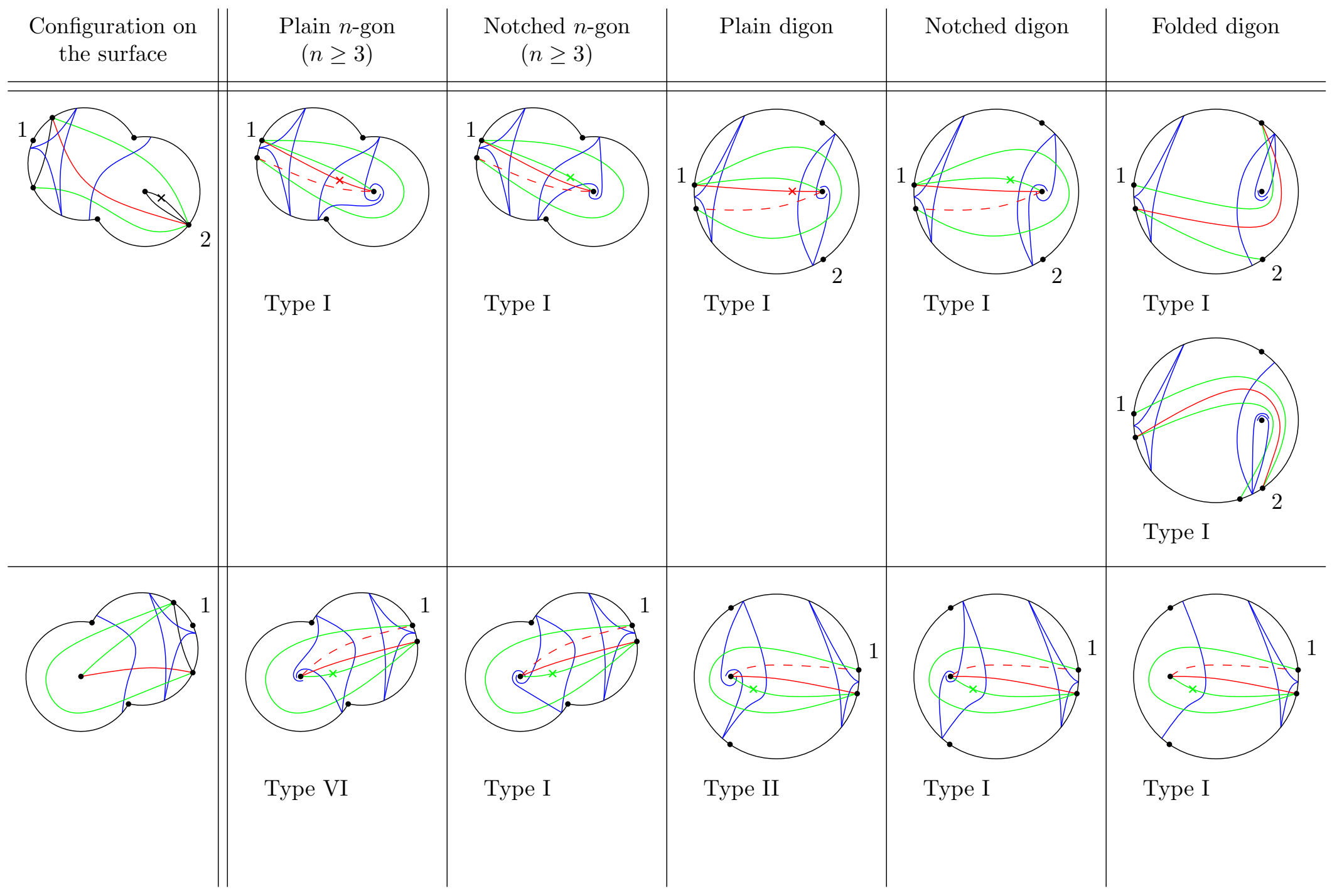




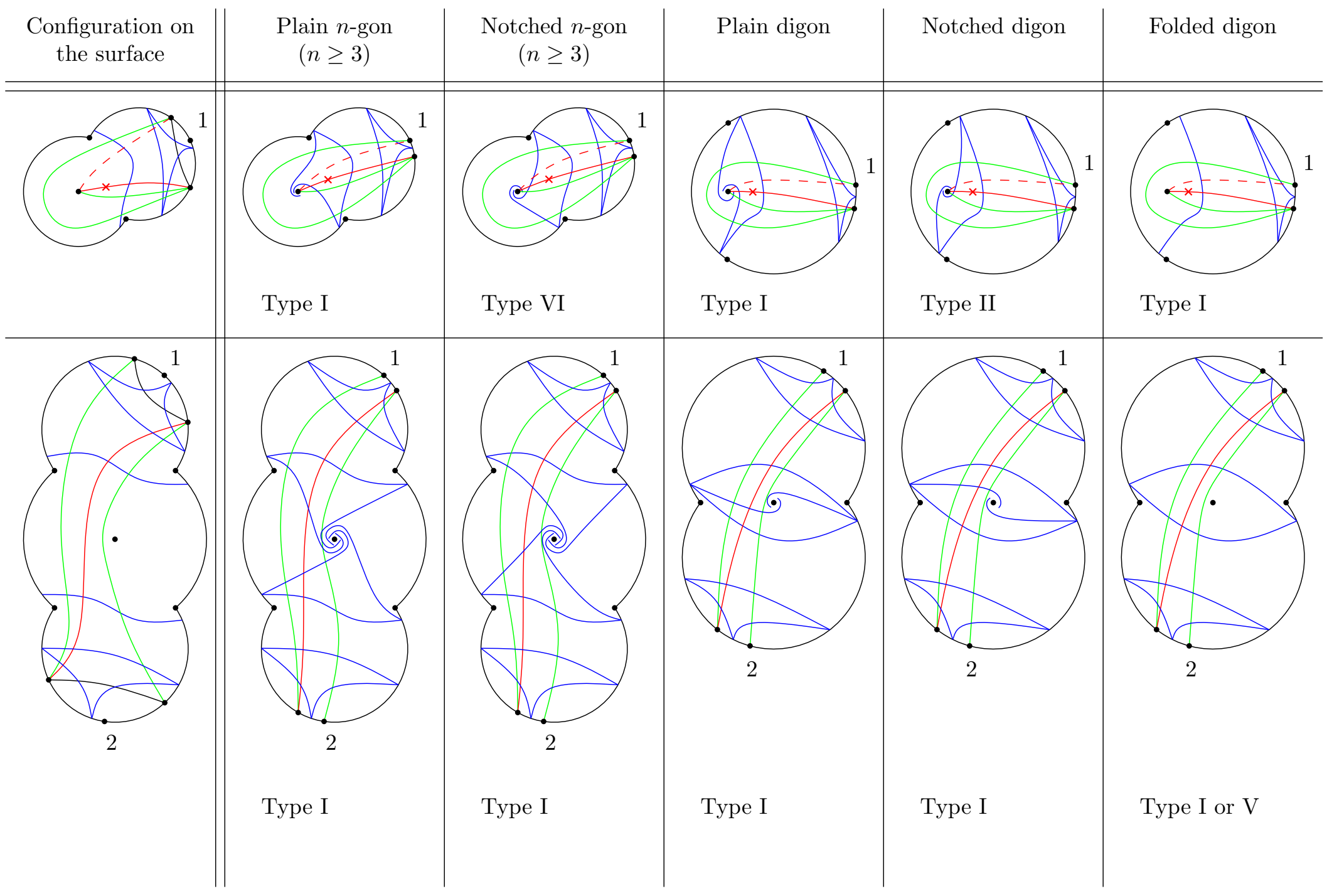




\section{B The sets $\mathcal{X}(Z)$ and $\mathcal{W}(Z)$ for the exceptional types.}

For exceptional types we obtain a description of $\mathcal{X}(Z)$ by direct inspection using [35, 38]. Similarly we obtain $\mathcal{W}(Z)$ and check Theorems $1.1,1.3$, and 1.4 .

\section{B.1 Type $G_{2}$}

\section{$\Longleftrightarrow$}

Figure B.1: The only diagram in $\mathcal{X}\left(G_{2}\right)$.

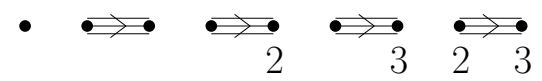

Figure B.2: The set $\mathcal{W}\left(G_{2}\right)$.

\section{B.2 Type $F_{4}$}

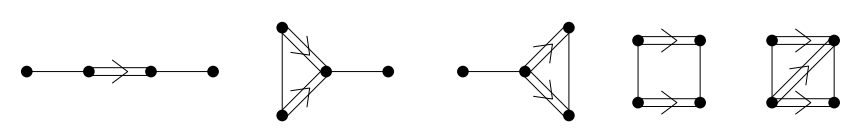

Figure B.3: Diagrams in $\mathcal{X}\left(F_{4}\right)$.
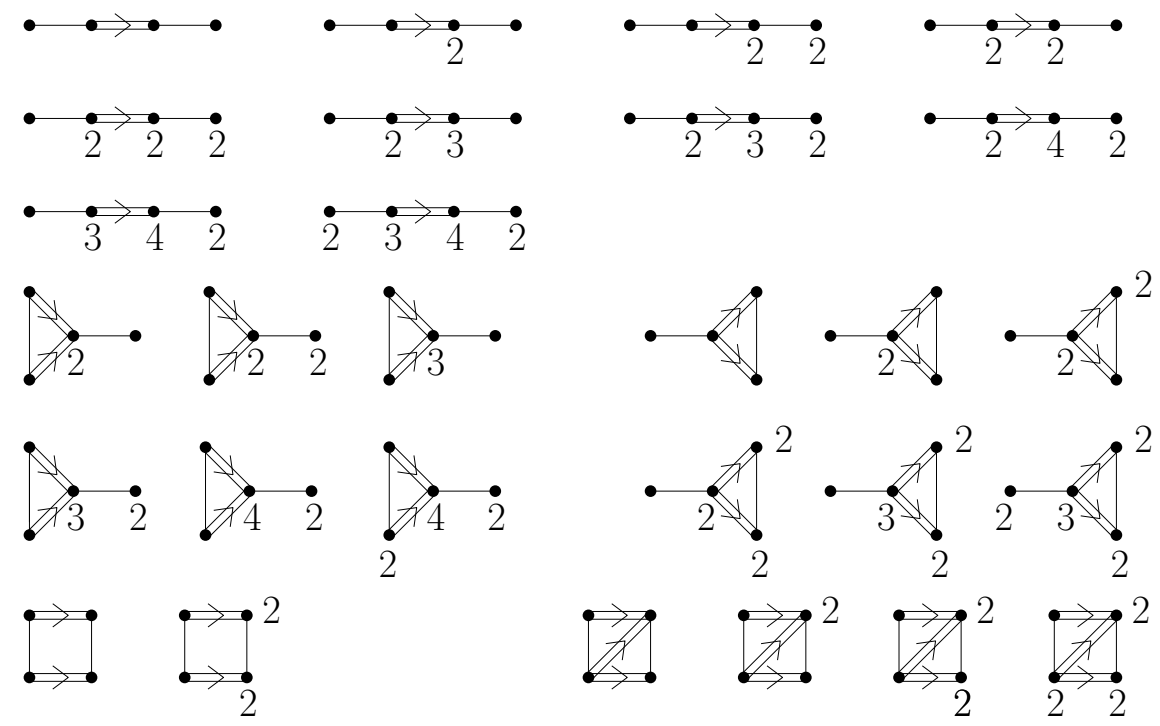

Figure B.4: The set $\mathcal{W}\left(F_{4}\right)$ consists of the above weighed diagrams together with all the elements of $\mathcal{W}\left(B_{3}\right)$ and $\mathcal{W}\left(C_{3}\right)$. 


\section{B.3 Type $E_{6}$}

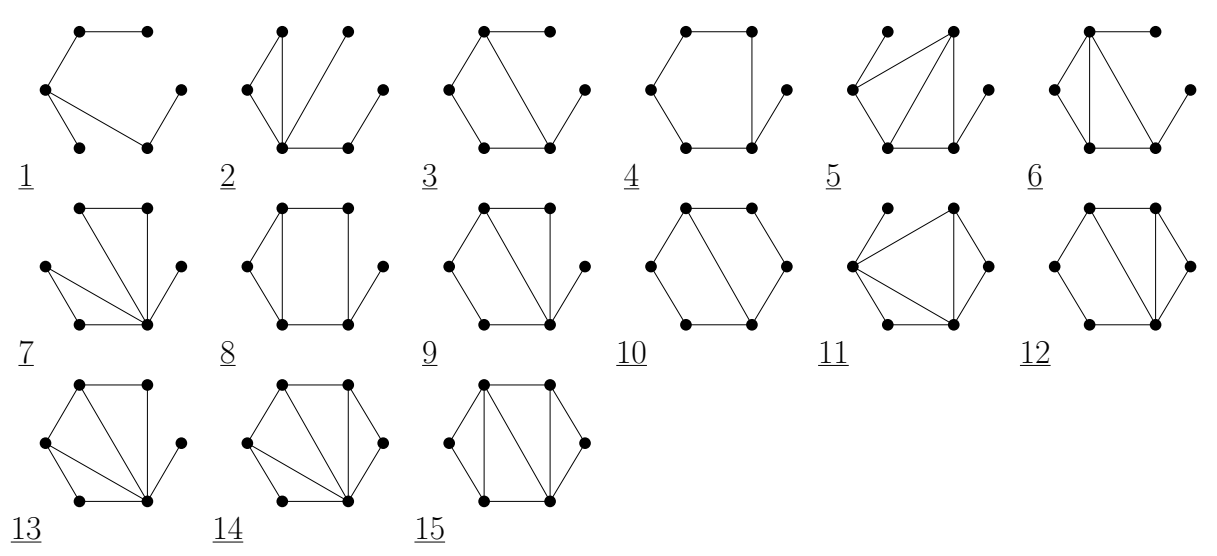

Figure B.5: Diagrams in $\mathcal{X}\left(E_{6}\right)$.
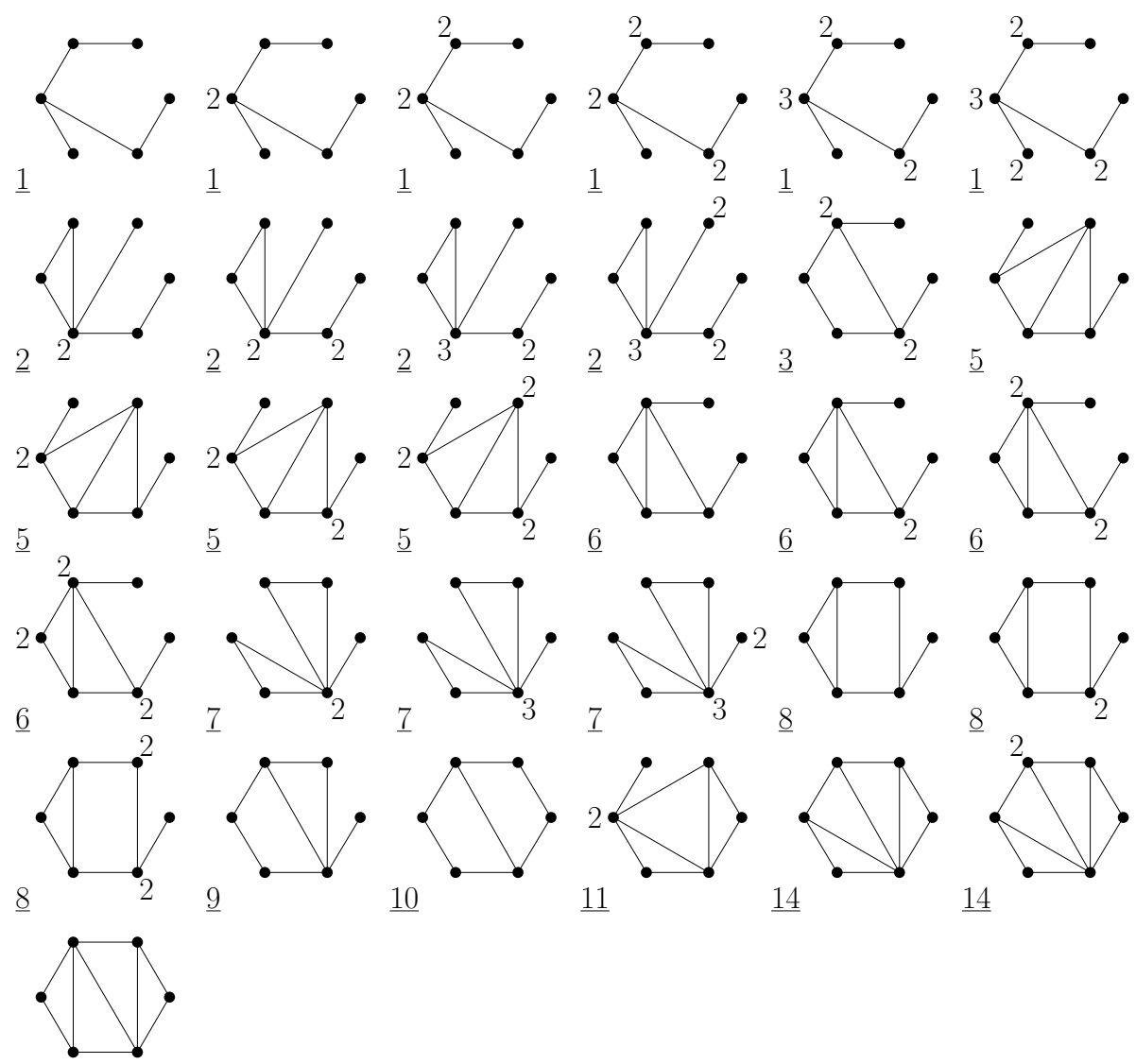

15

Figure B.6: The set $\mathcal{W}\left(E_{6}\right)$ consists of the above weighted diagrams together with all the elements of $\mathcal{W}\left(A_{5}\right)$ and $\mathcal{W}\left(D_{5}\right)$. 


\section{B.4 Type $E_{7}$}
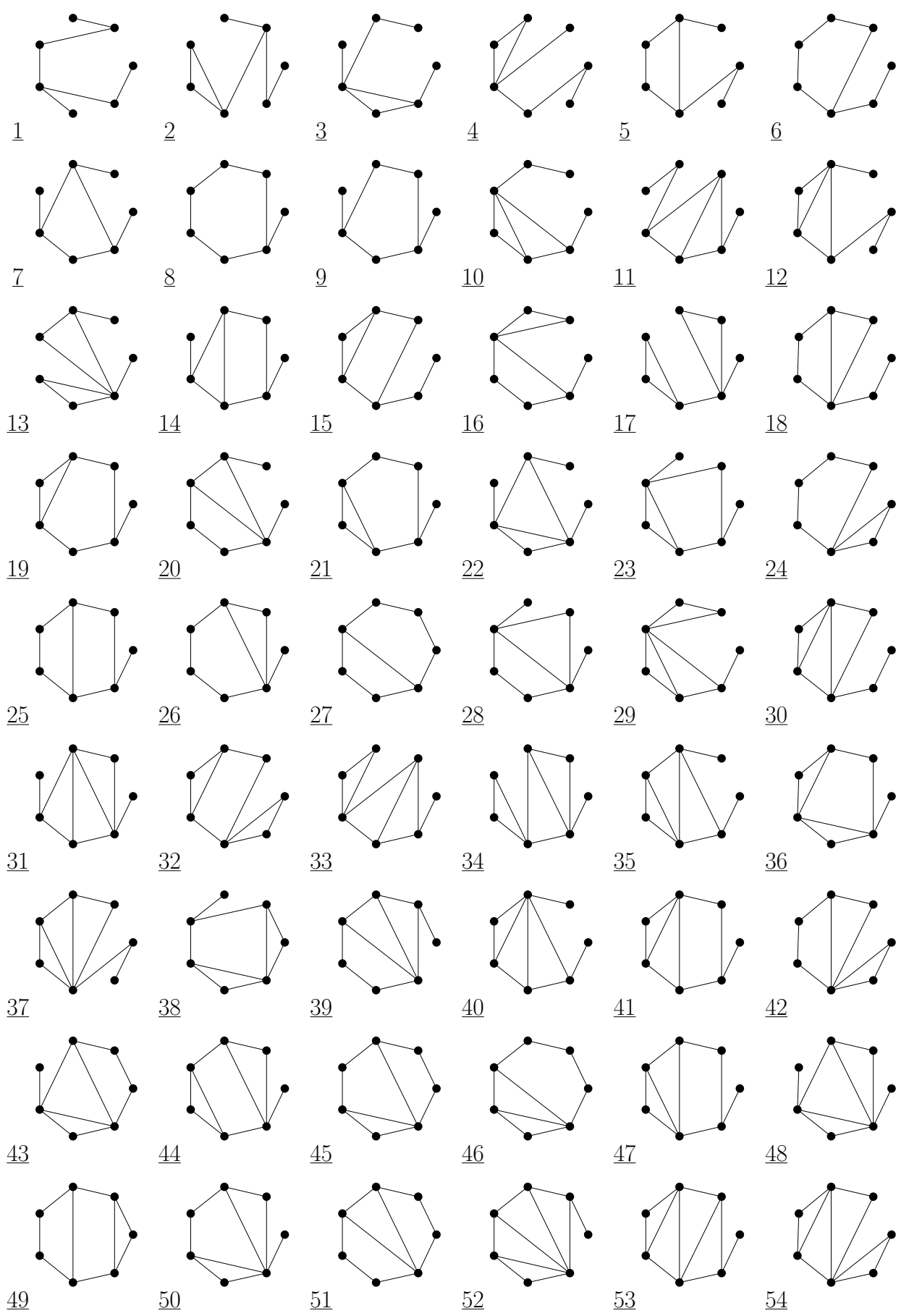

Figure B.7: Diagrams in $\mathcal{X}\left(E_{7}\right)$. 

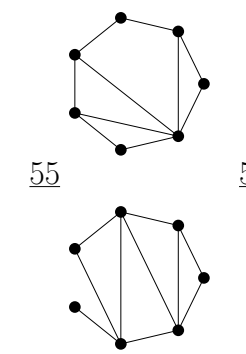

$\underline{61}$

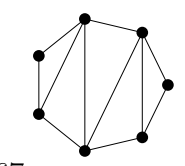

$\underline{67}$
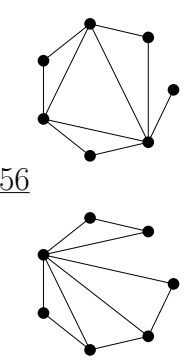

$\underline{62}$

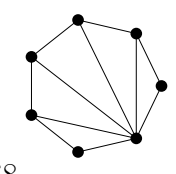

$\underline{63}$
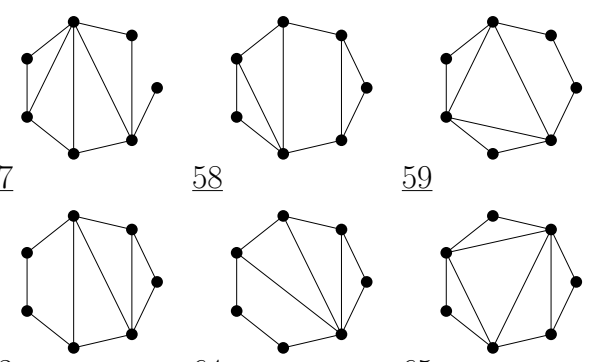

$\underline{60}$
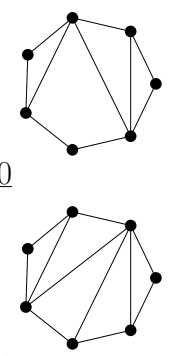

$\underline{66}$

Figure B.8: Diagrams in $\mathcal{X}\left(E_{7}\right)$ (continued).
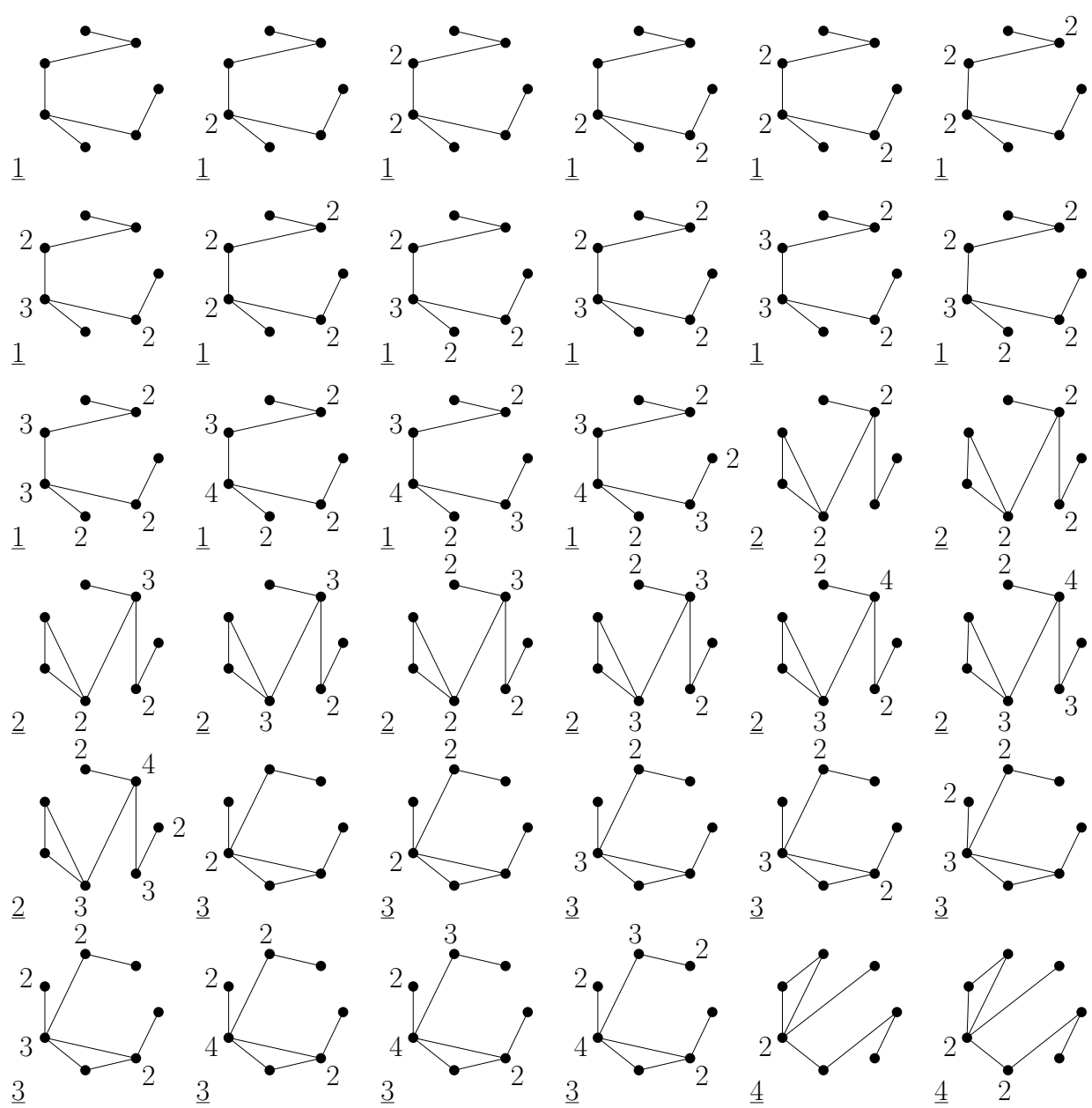

Figure B.9: The set $\mathcal{W}\left(E_{7}\right)$ consists of the above weighted diagrams and all the elements of $\mathcal{W}\left(A_{6}\right), \mathcal{W}\left(D_{6}\right)$, and $\mathcal{W}\left(E_{6}\right)$. 

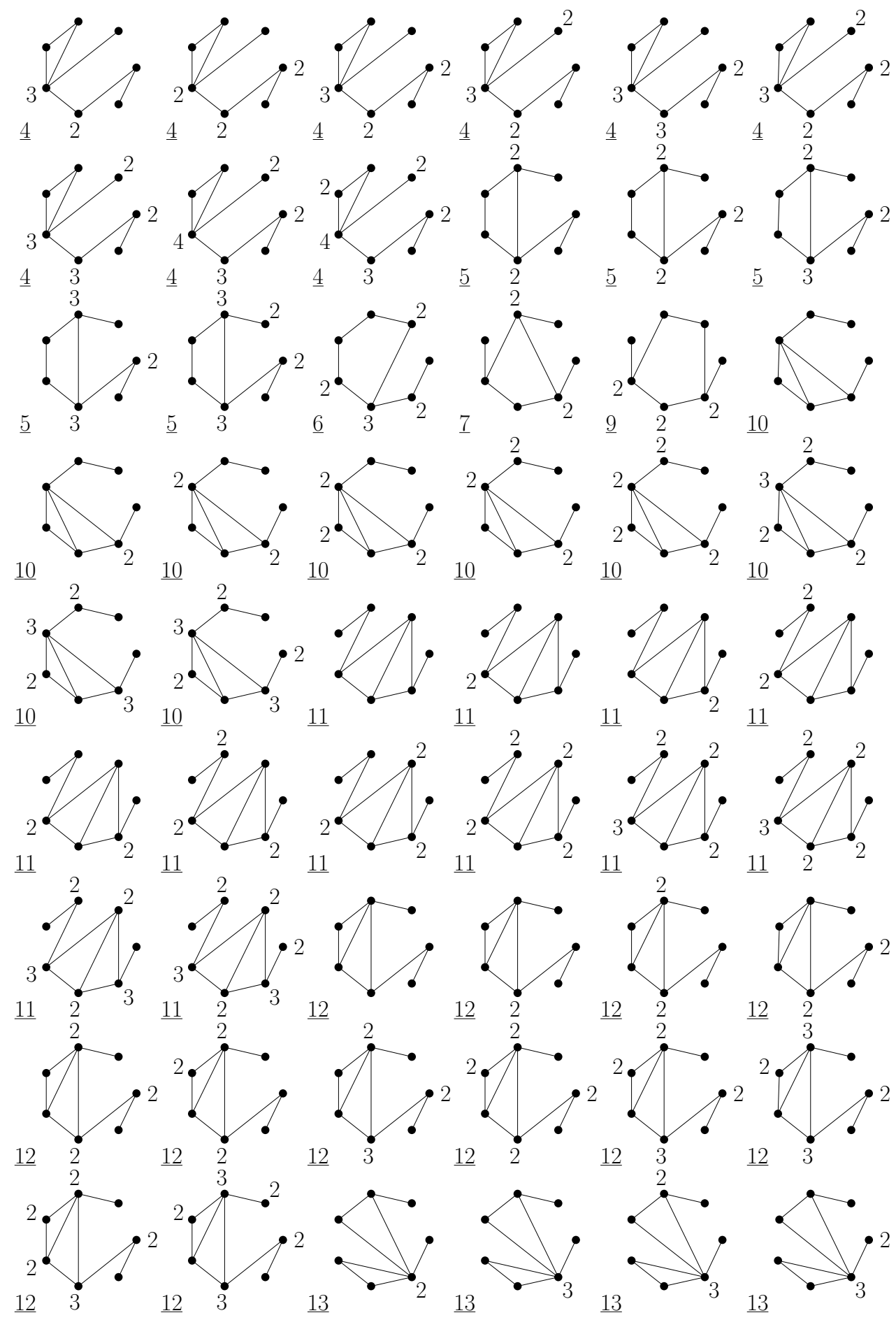

Figure B.10: The set $\mathcal{W}\left(E_{7}\right)$ (continued). 

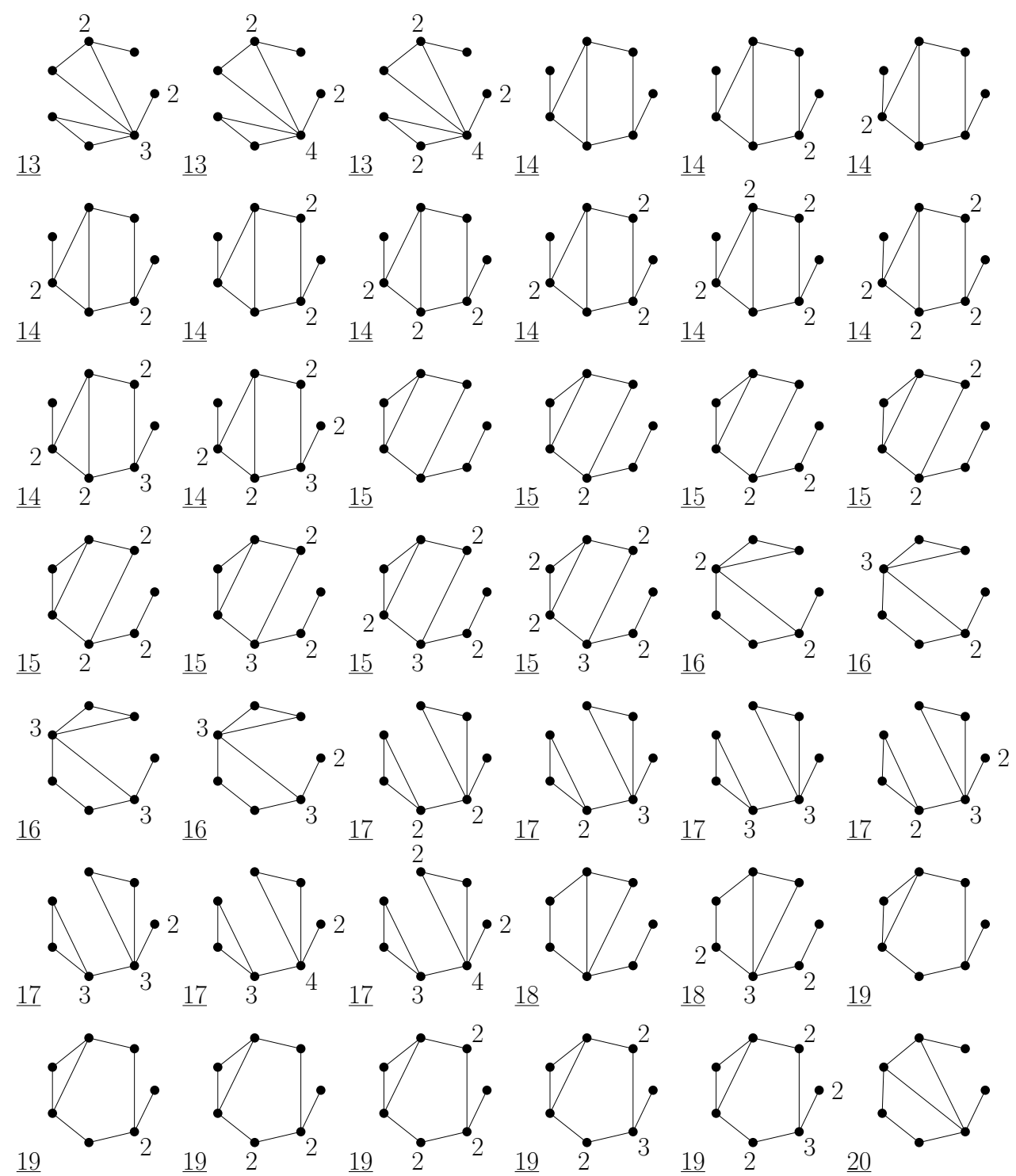

$\underline{19}$
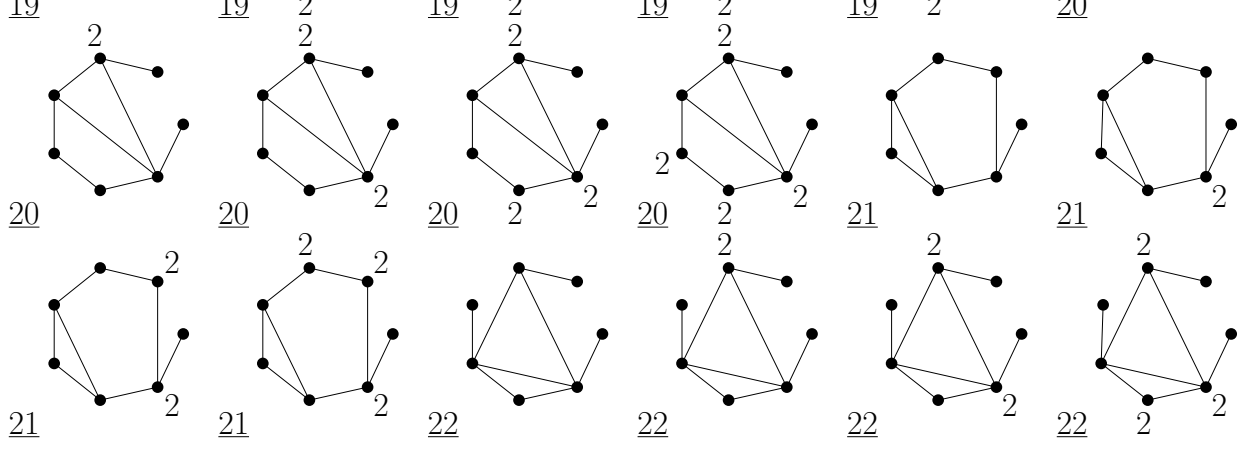

Figure B.11: The set $\mathcal{W}\left(E_{7}\right)$ (continued). 

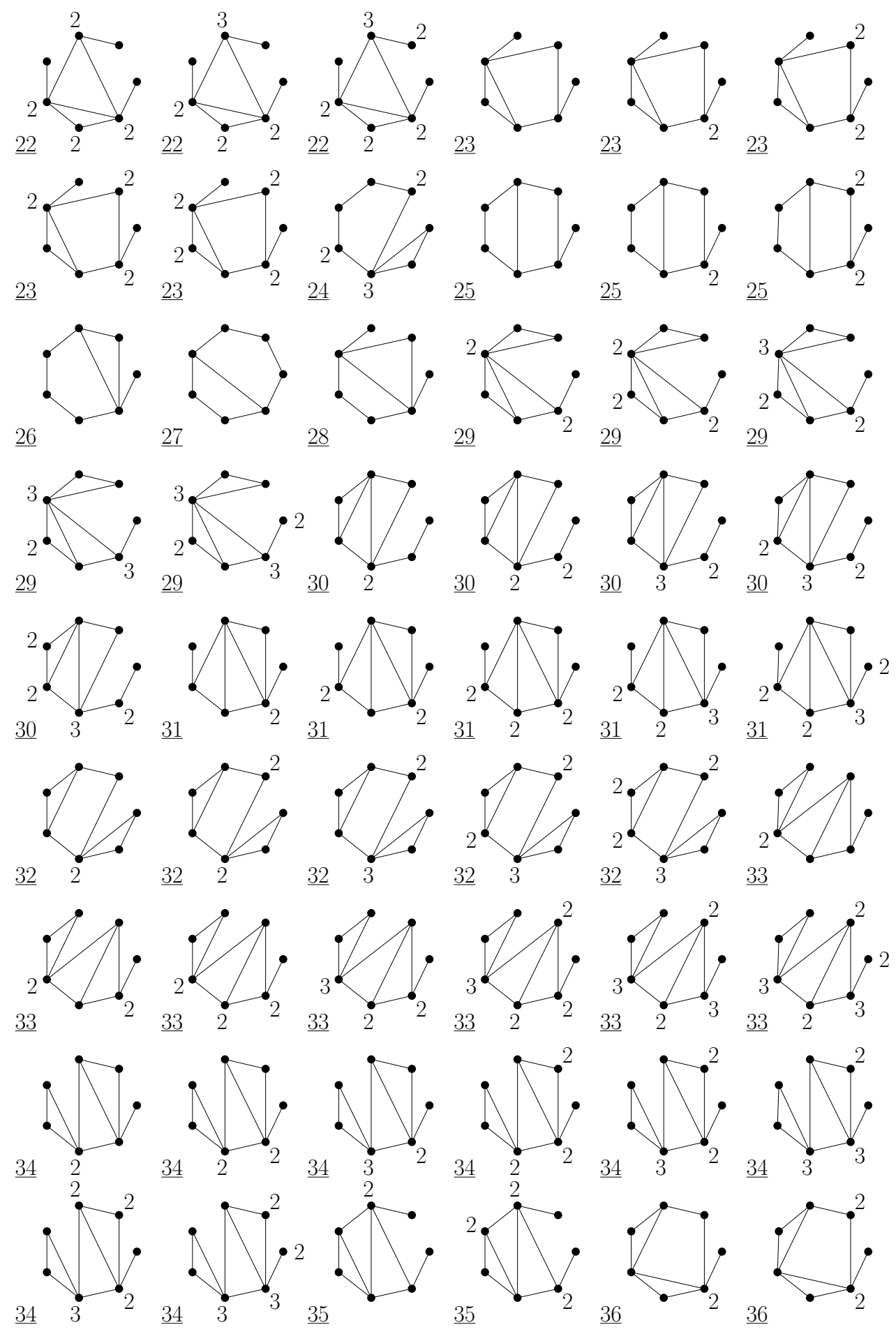

Figure B.12: The set $\mathcal{W}\left(E_{7}\right)$ (continued). 

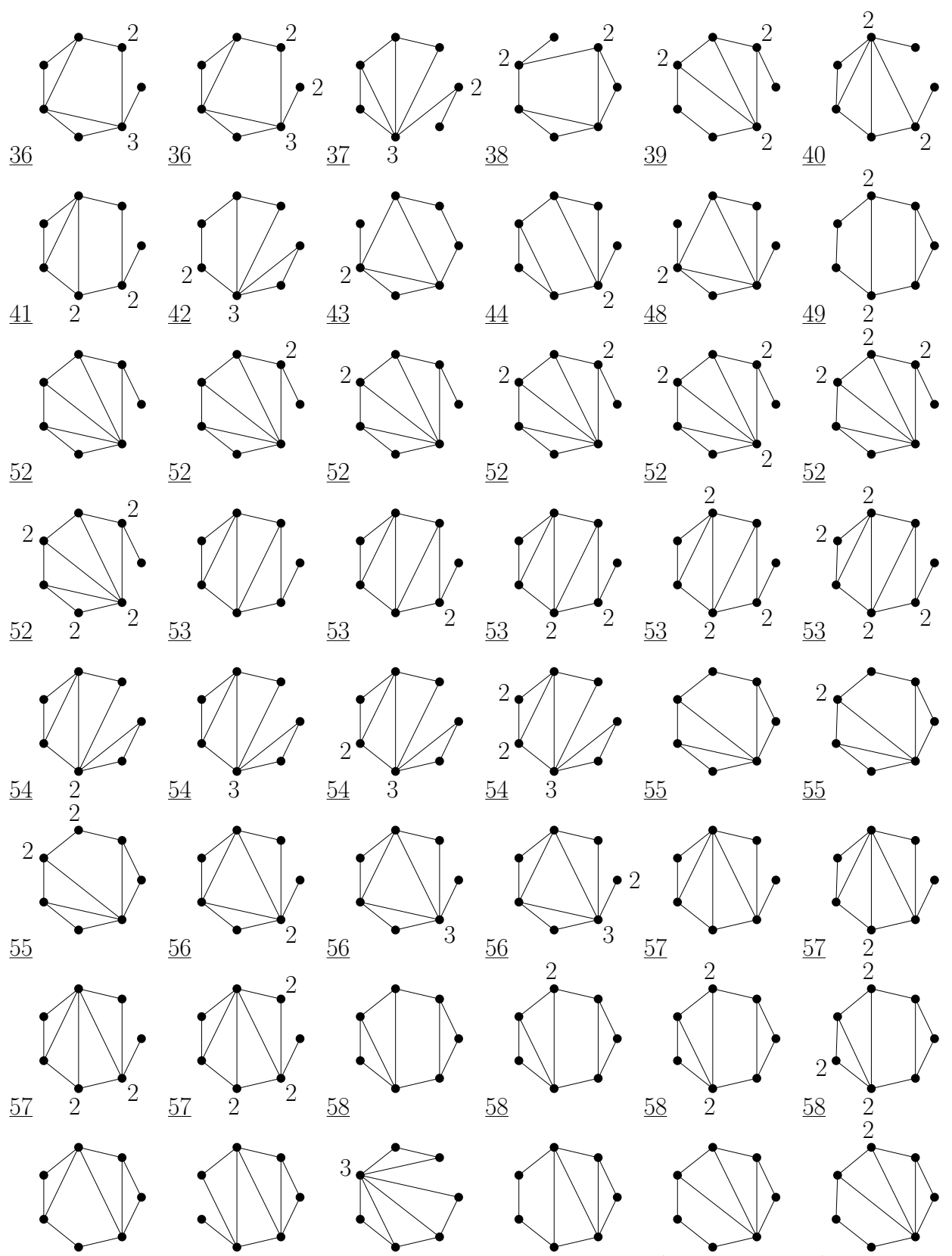

$\underline{60}$

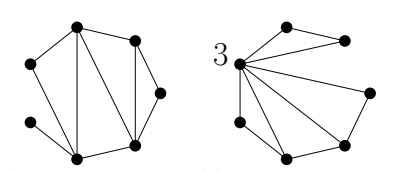

$\underline{61}$

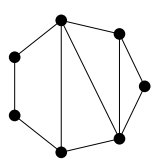

$\underline{63}$

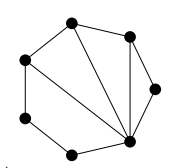

$\underline{64}$
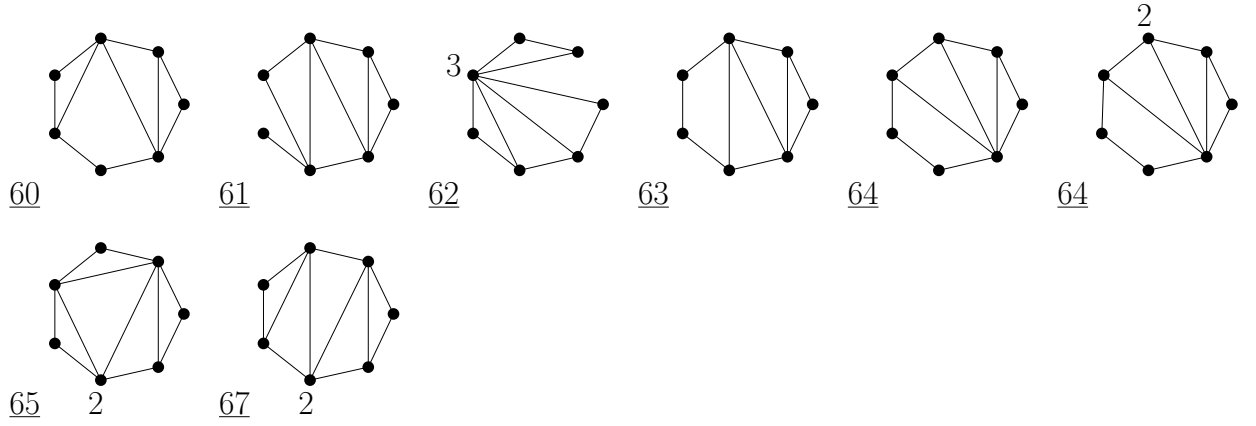

Figure B.13: The set $\mathcal{W}\left(E_{7}\right)$ (continued). 


\section{B.5 Type $E_{8}$}
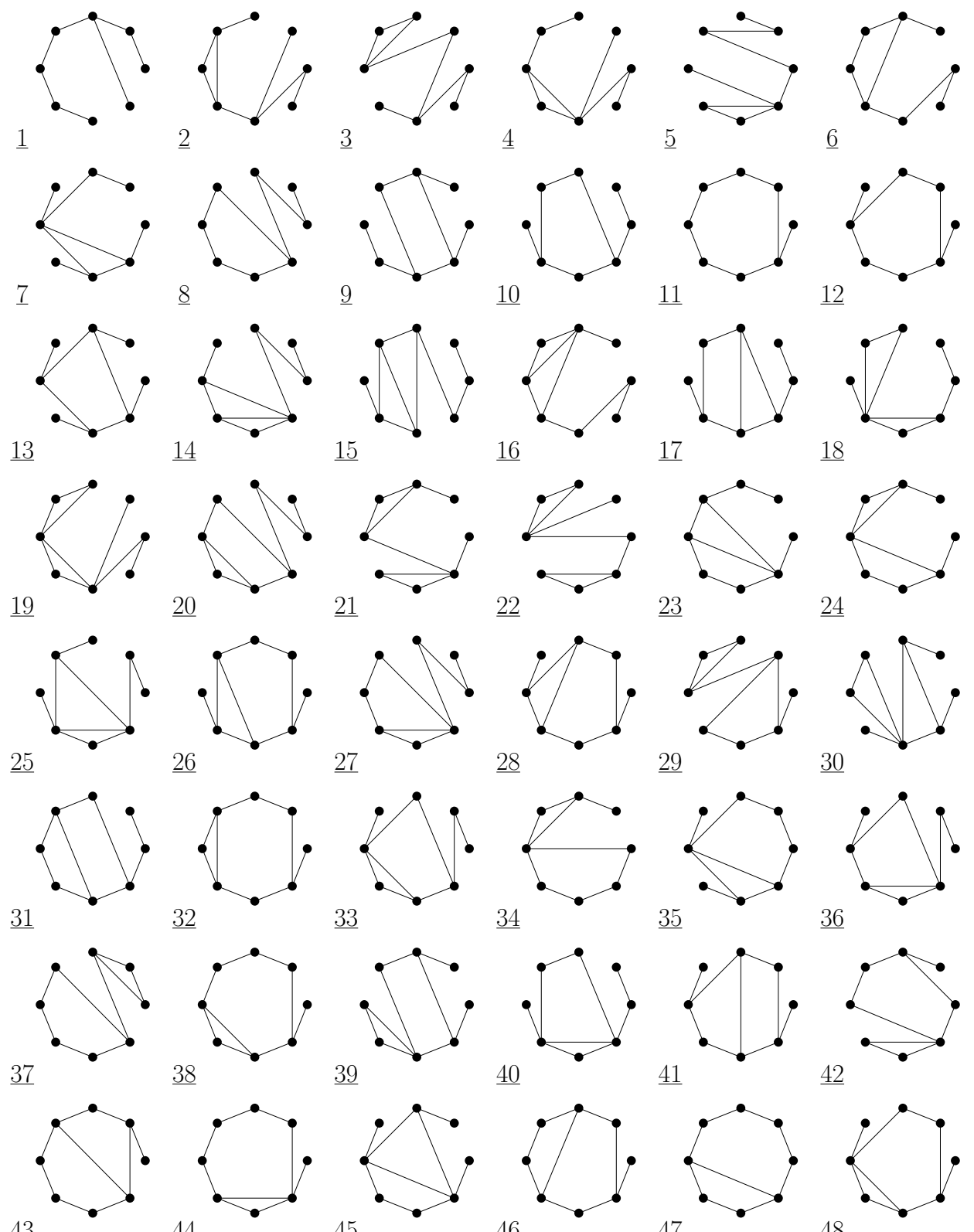

$\underline{41}$
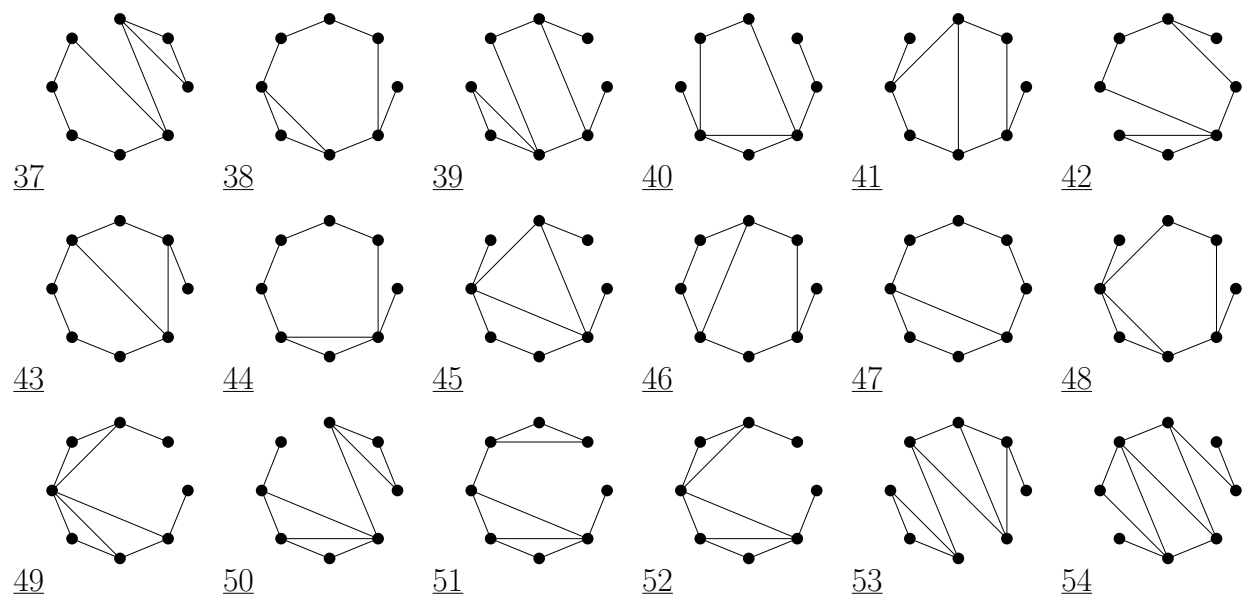

45
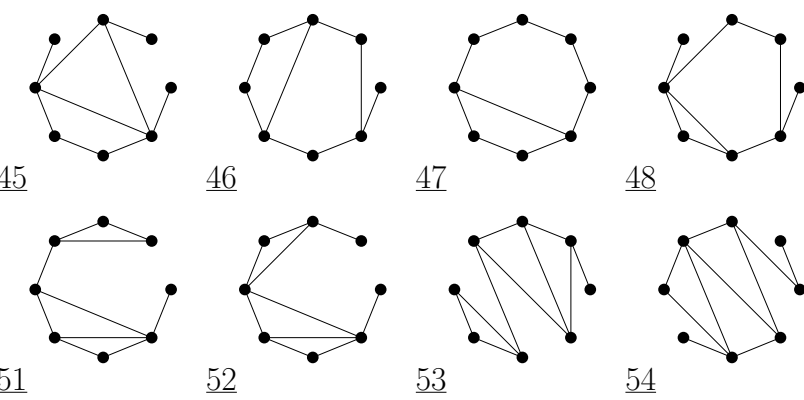

$\underline{47} \quad \underline{48}$

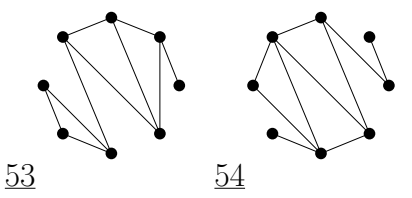

Figure B.14: Diagrams in $\mathcal{X}\left(E_{8}\right)$. 

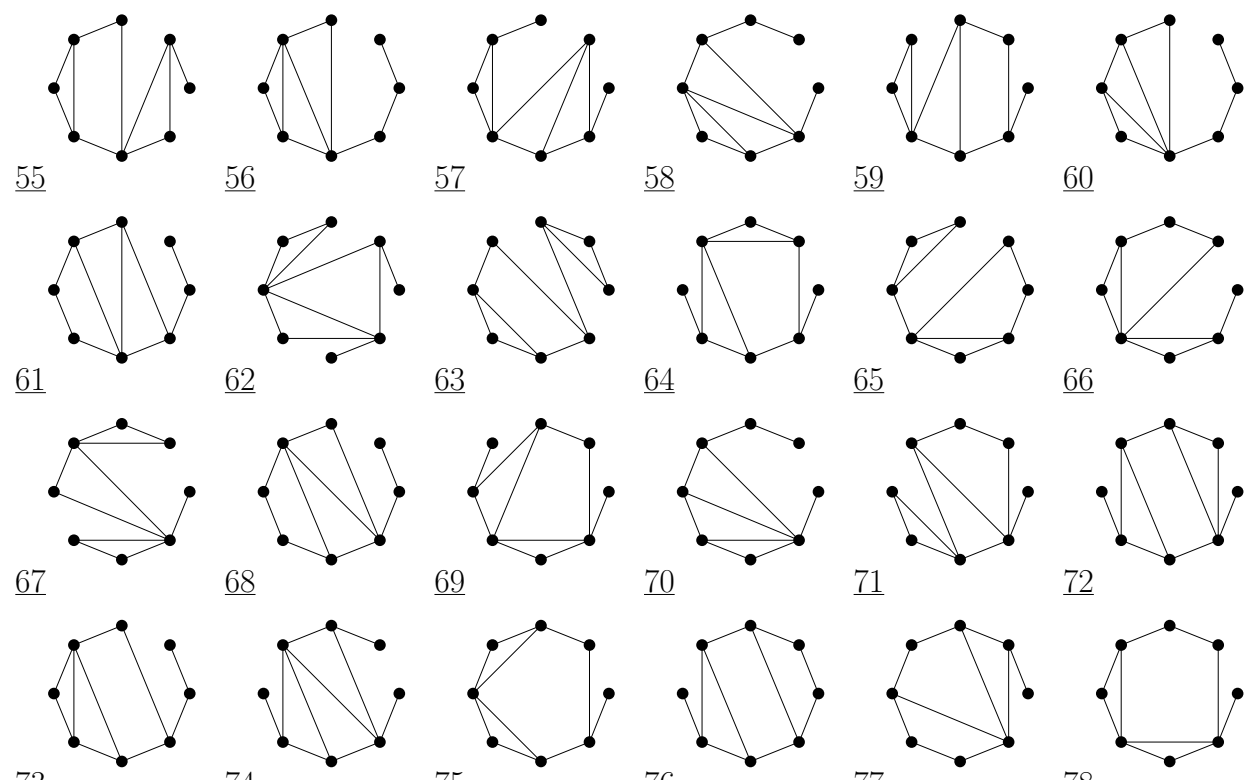

$\underline{73}$
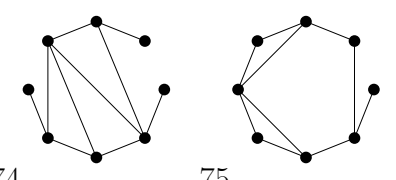

$\underline{70}$

$\underline{71}$
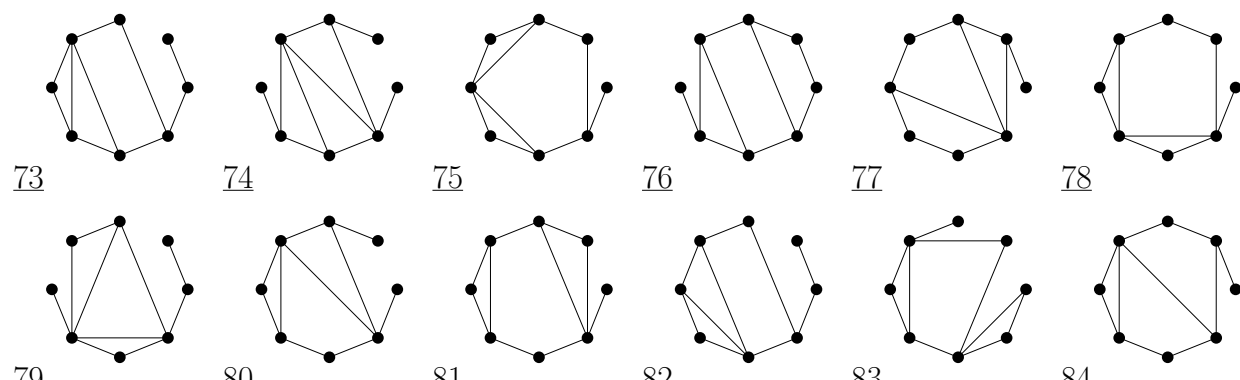

$\underline{78}$

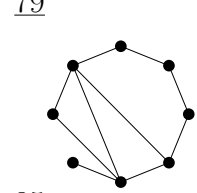

$80 \quad 81$

$\underline{82}$

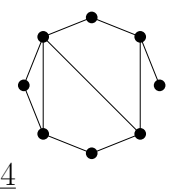

$\underline{85}$
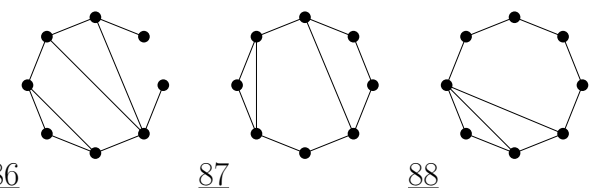

$\underline{88}$

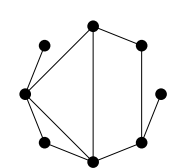

$\underline{87} \quad \underline{88}$

$\underline{89}$
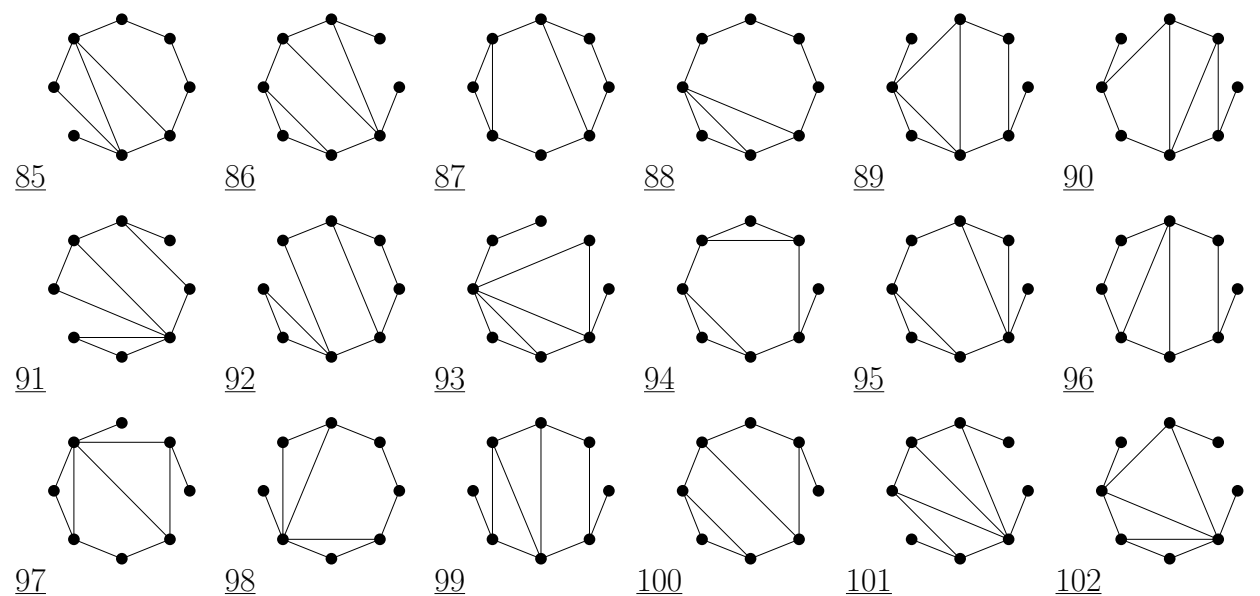

$\underline{96}$

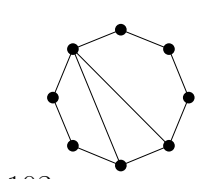

$\underline{103}$
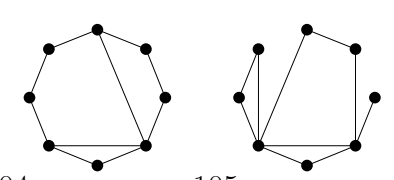

100
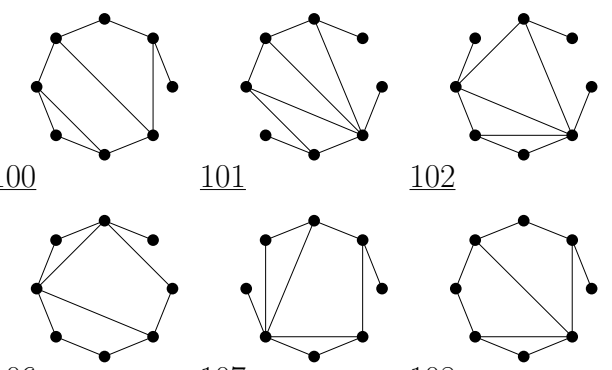

$\underline{106}$

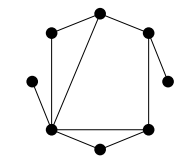

$\underline{107}$

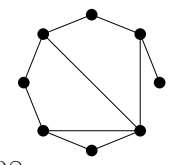

Figure B.15: Diagrams in $\mathcal{X}\left(E_{8}\right)$ (continued). 


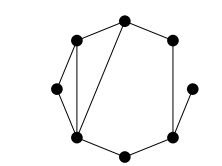

109

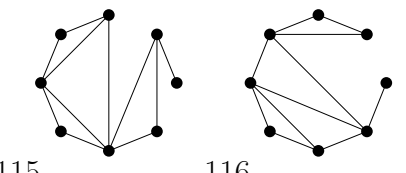

115

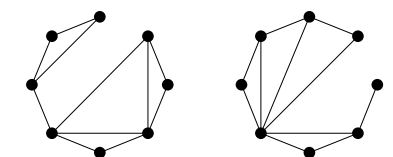

121

122

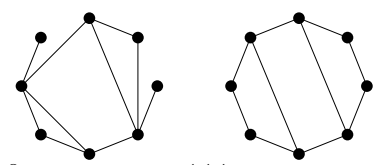

111

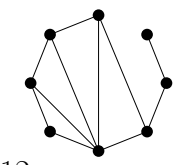

112

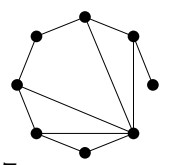

117

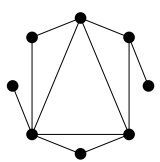

118
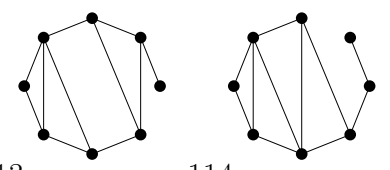

114

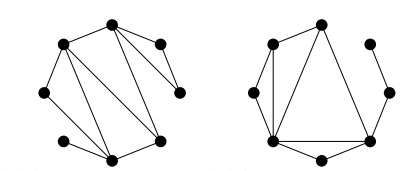

119

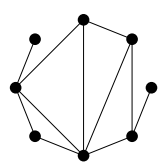

124

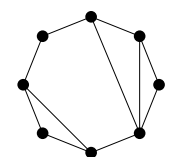

$\underline{125}$
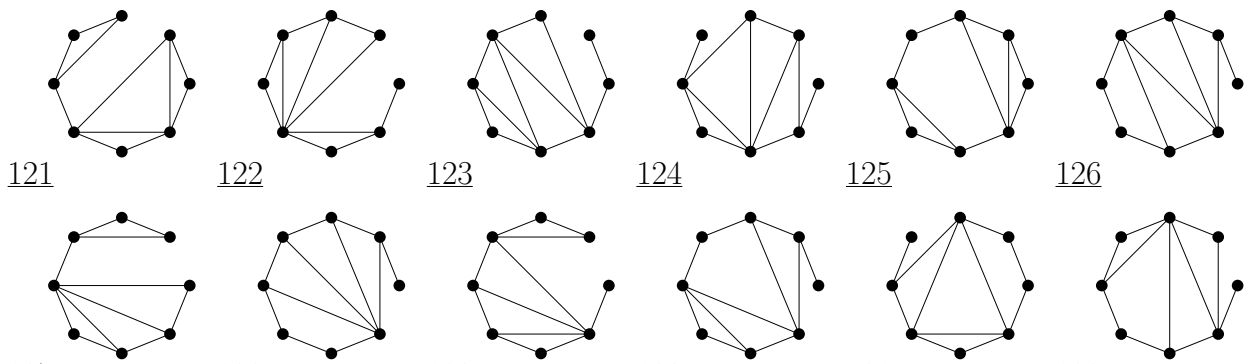

127
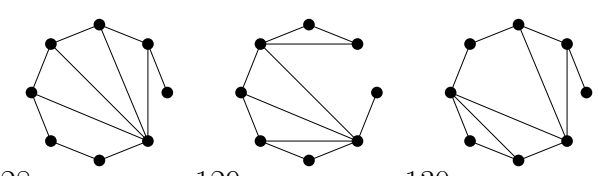

$\underline{130}$

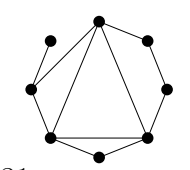

$\underline{126}$
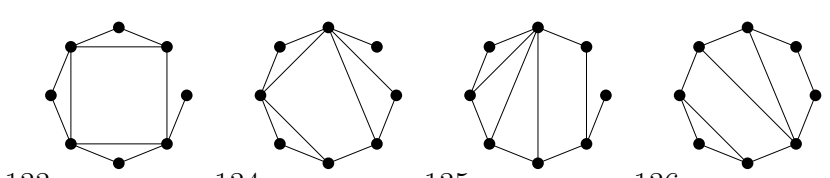

131

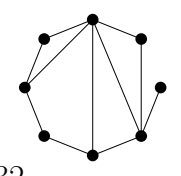

$\underline{133}$

134

135

$\underline{136}$

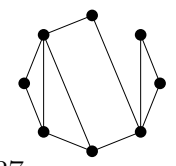

132

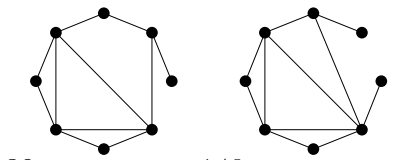

$\underline{139}$

$\underline{140}$

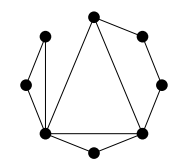

141

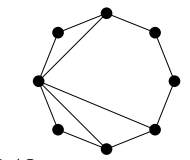

137
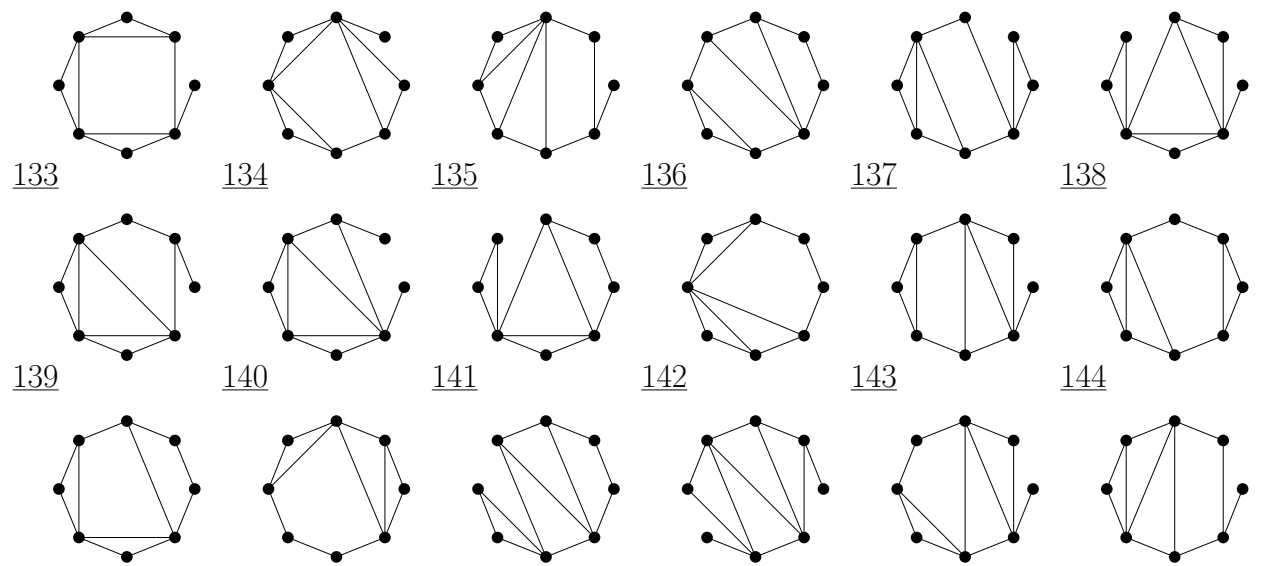

142

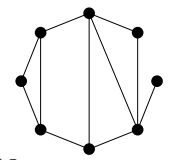

$\underline{143}$

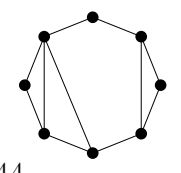

$\underline{145}$

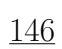

147

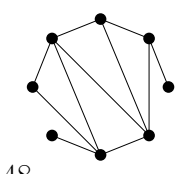

148
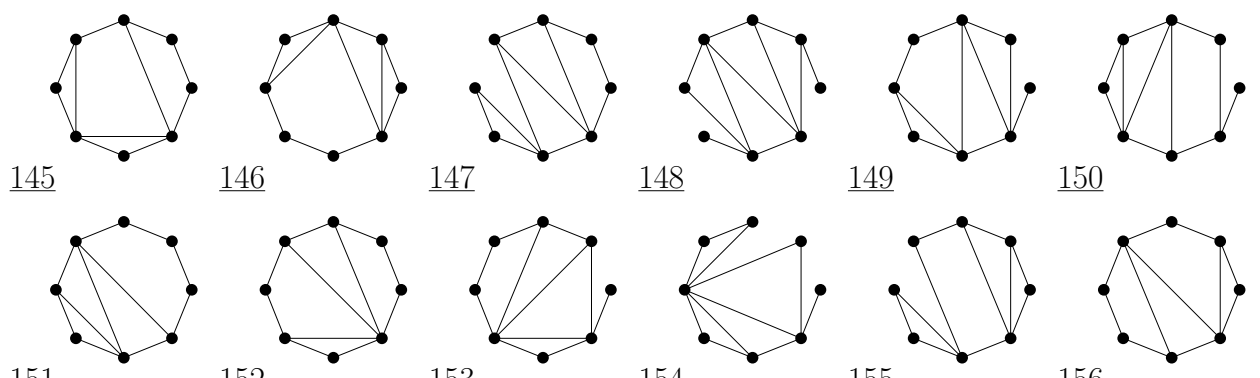

$\underline{149}$

150

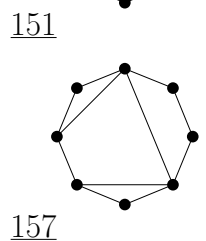

152

153

$\underline{154}$
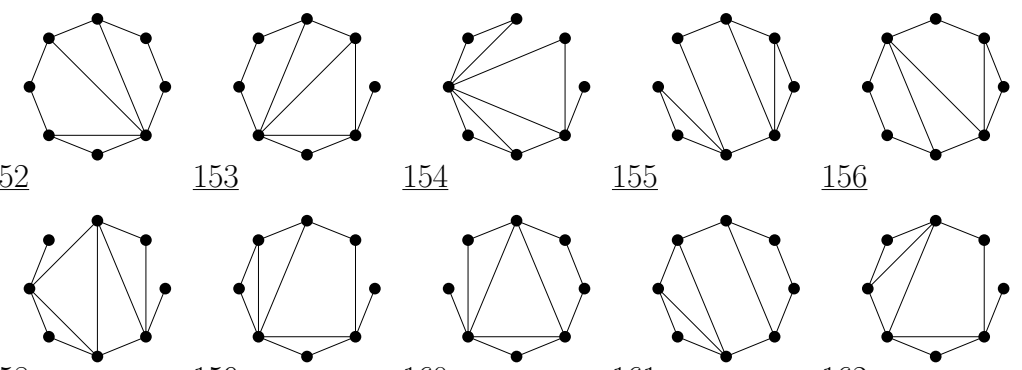

$\underline{158}$

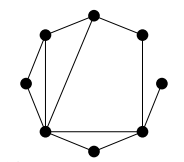

$\underline{159}$

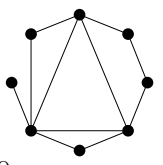

$\underline{160}$

$\underline{156}$

Figure B.16: Diagrams in $\mathcal{X}\left(E_{8}\right)$ (continued). 

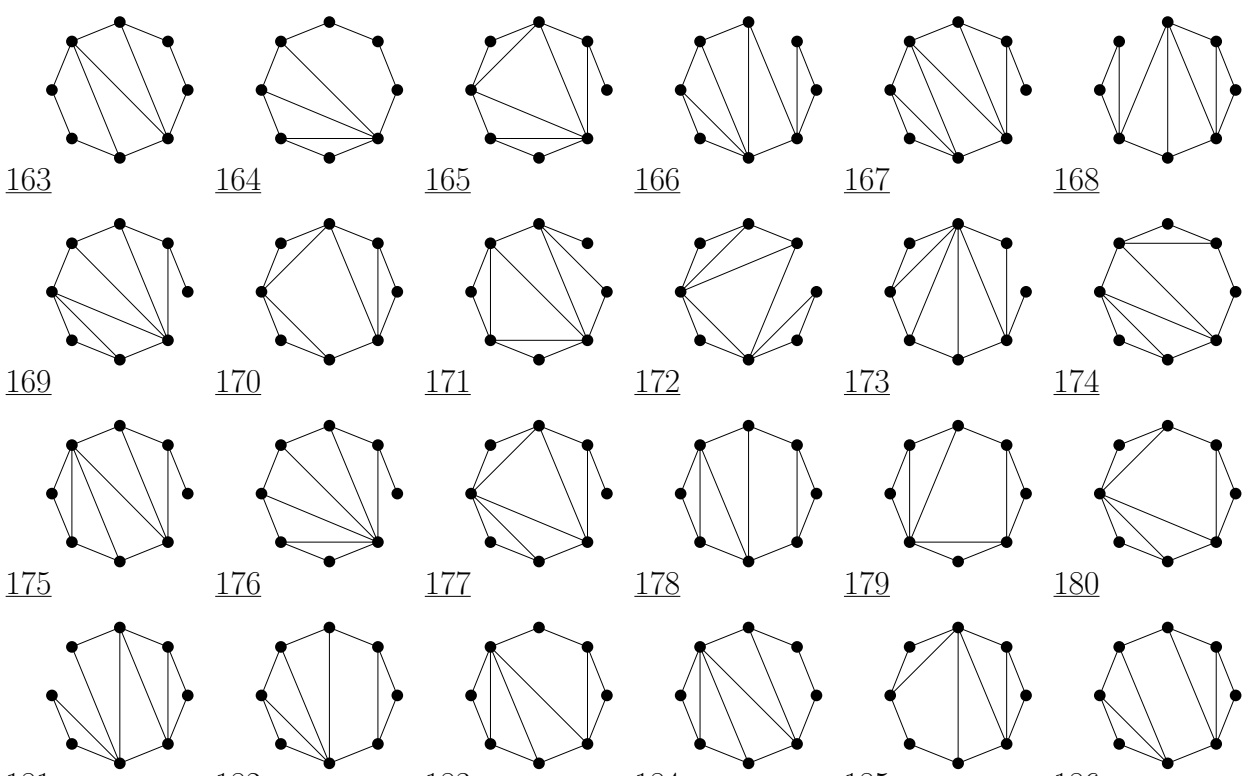

170 171

172

$\underline{173}$

174

$\underline{181}$
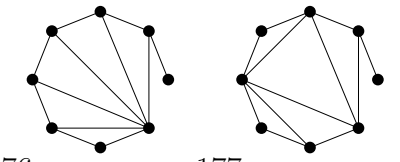

$\underline{177}$

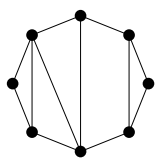

$\underline{178}$

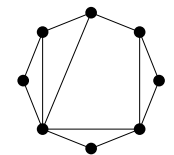

$\underline{179}$

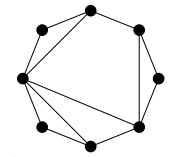

$\underline{180}$
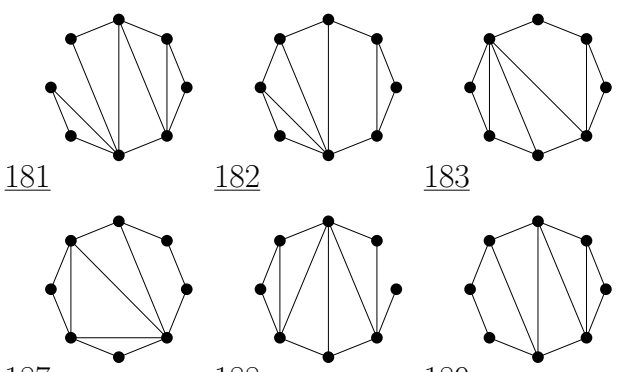

$\underline{183}$
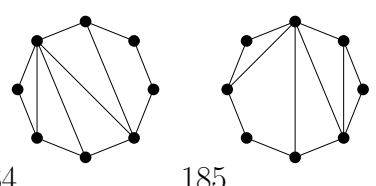

$\underline{185}$
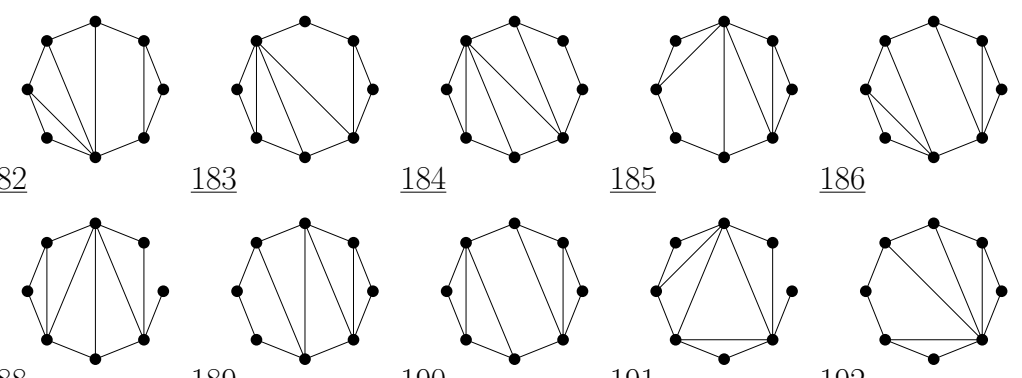

$\underline{186}$

$\underline{187}$

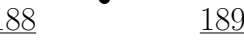

$\underline{190}$

$\underline{191}$
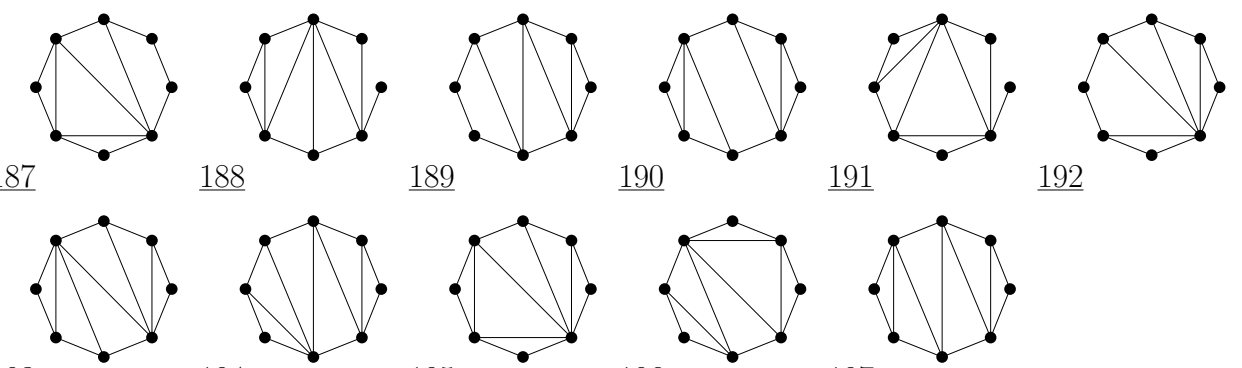

$\underline{193}$

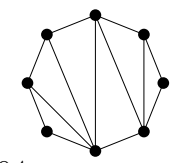

$\underline{194}$

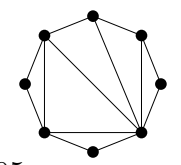

$\underline{195}$

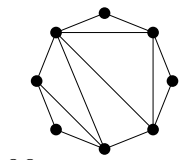

196

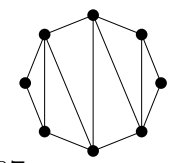

Figure B.17: Diagrams in $\mathcal{X}\left(E_{8}\right)$ (continued).
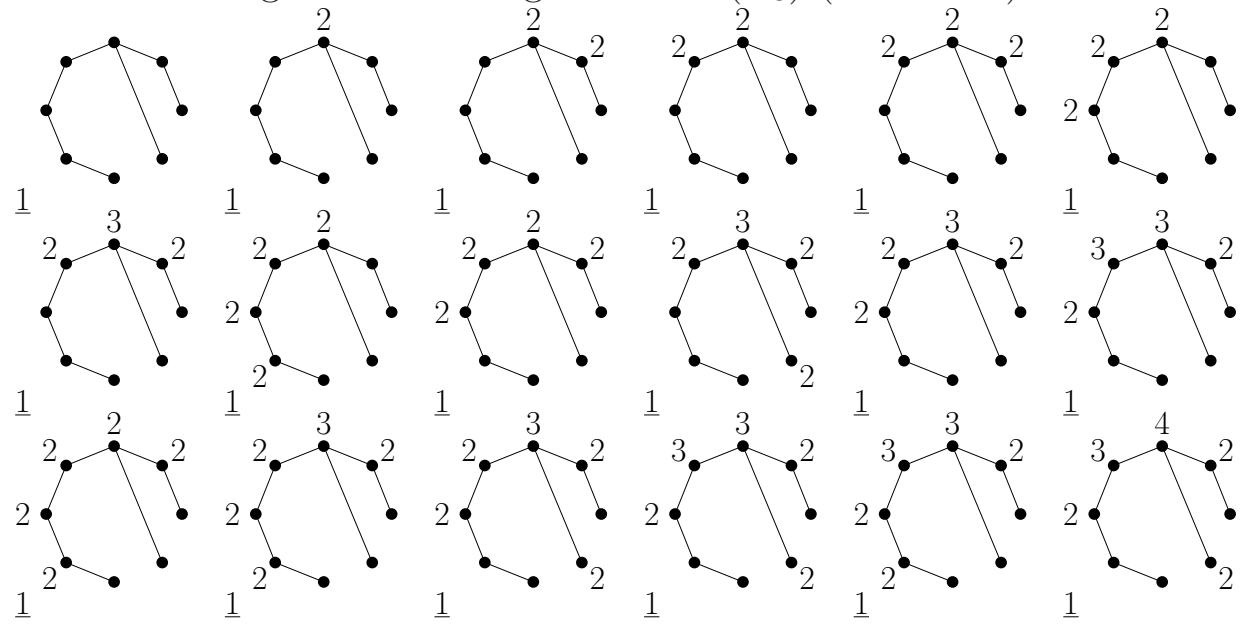

Figure B.18: The set $\mathcal{W}\left(E_{8}\right)$ consists of the above weighted diagrams and all the elements of $\mathcal{W}\left(A_{7}\right), \mathcal{W}\left(D_{7}\right)$, and $\mathcal{W}\left(E_{7}\right)$. 

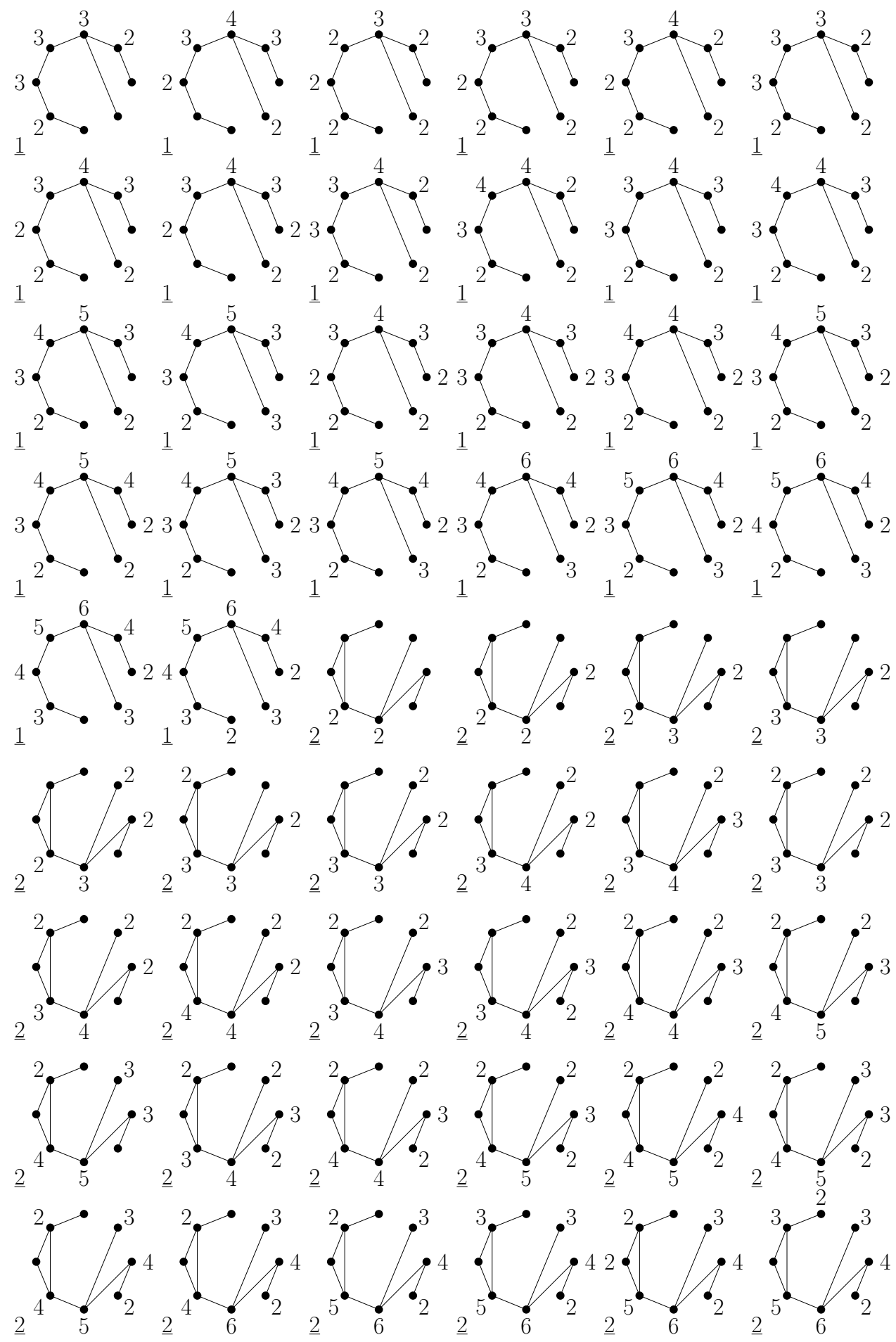

Figure B.19: The set $\mathcal{W}\left(E_{8}\right)$ (continued). 

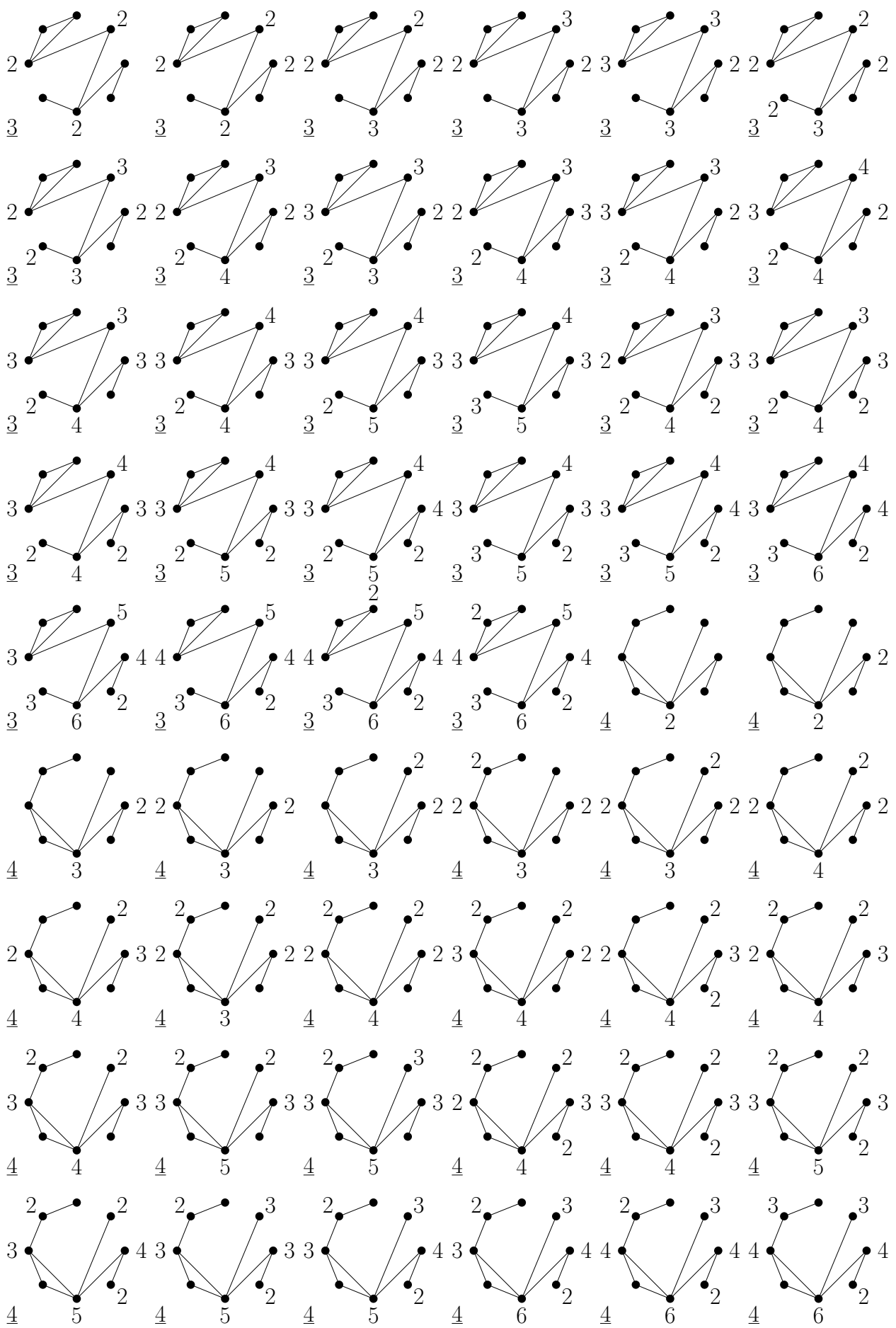

Figure B.20: The set $\mathcal{W}\left(E_{8}\right)$ (continued). 

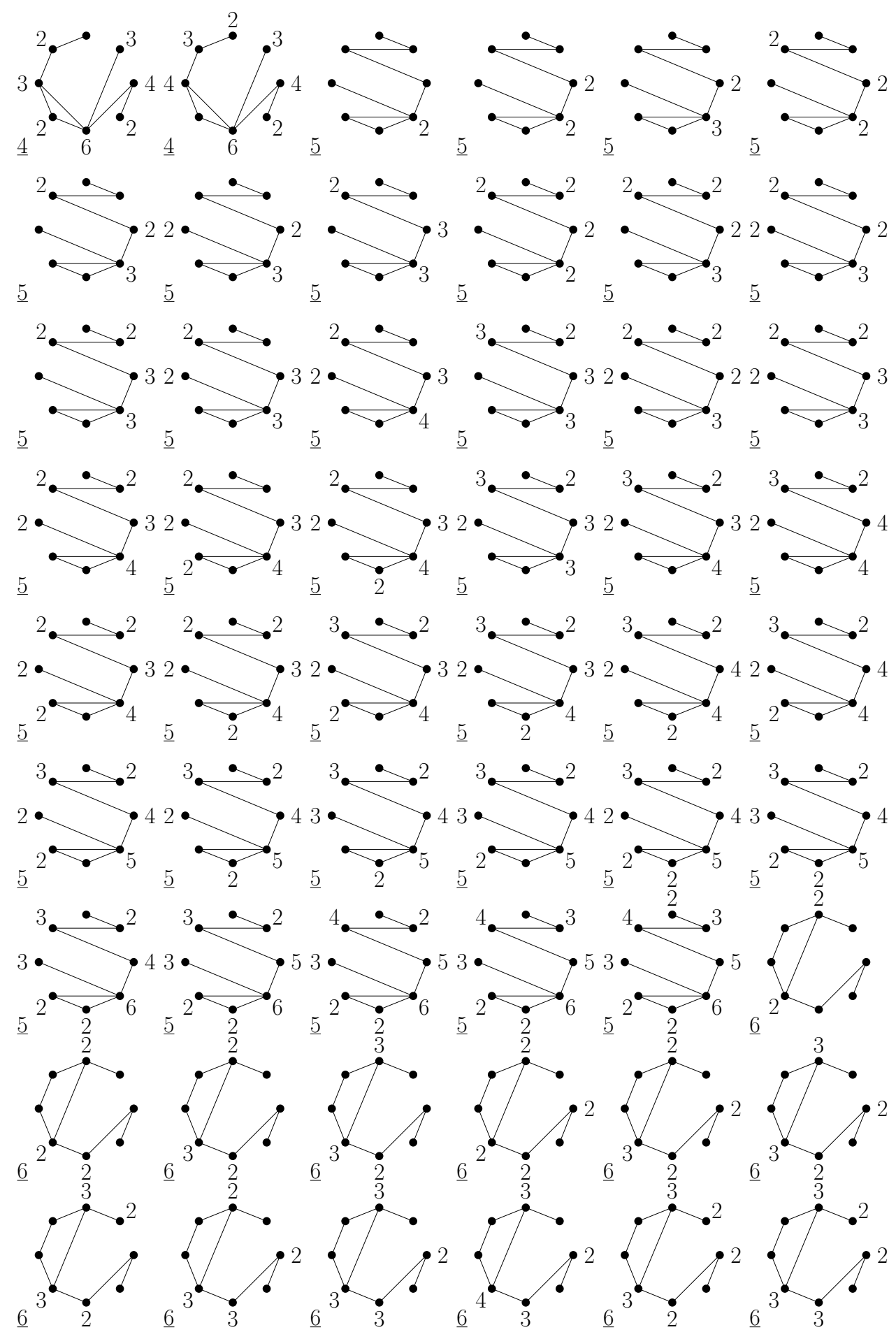

Figure B.21: The set $\mathcal{W}\left(E_{8}\right)$ (continued). 


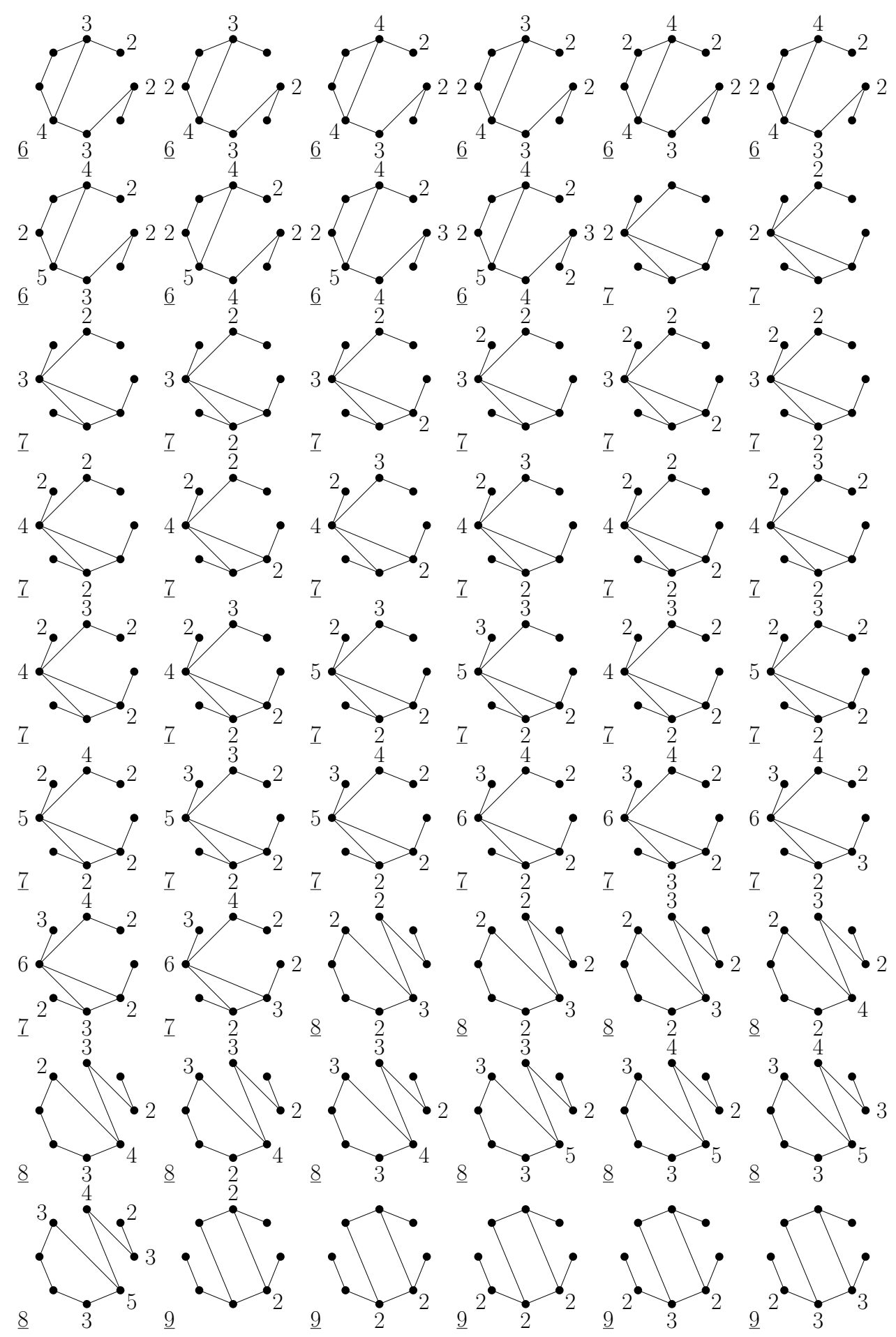

Figure B.22: The set $\mathcal{W}\left(E_{8}\right)$ (continued). 

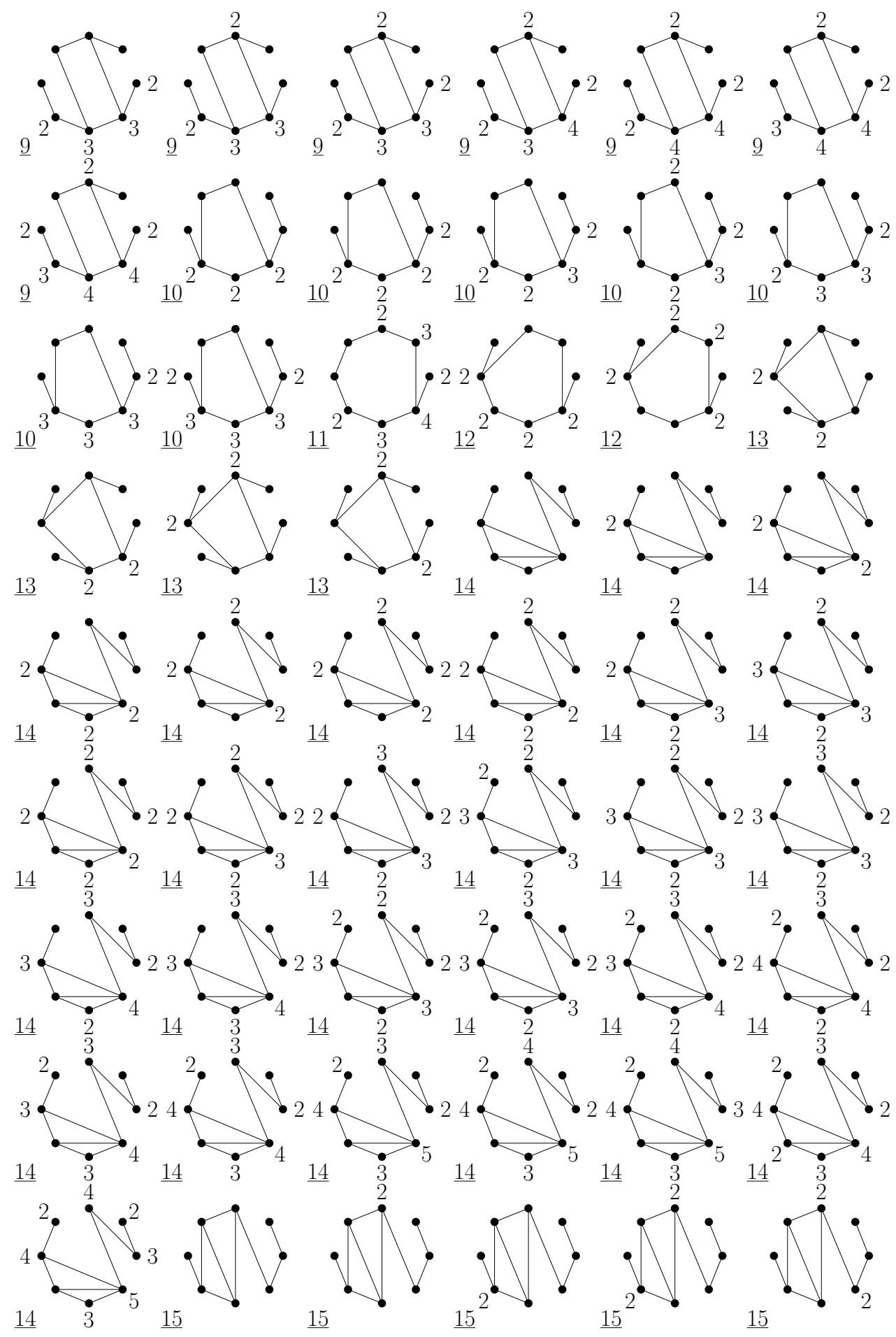

Figure B.23: The set $\mathcal{W}\left(E_{8}\right)$ (continued). 


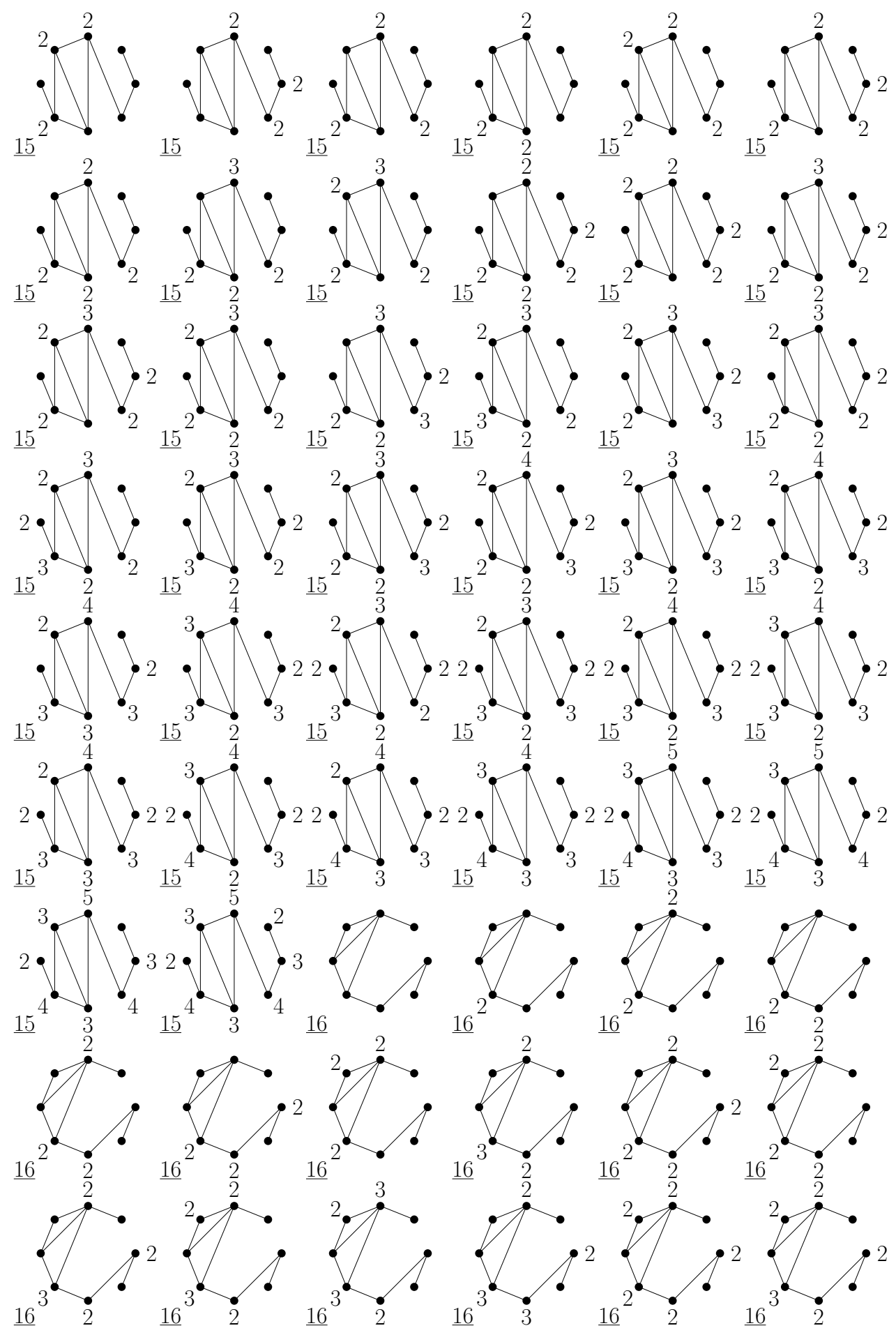

Figure B.24: The set $\mathcal{W}\left(E_{8}\right)$ (continued). 

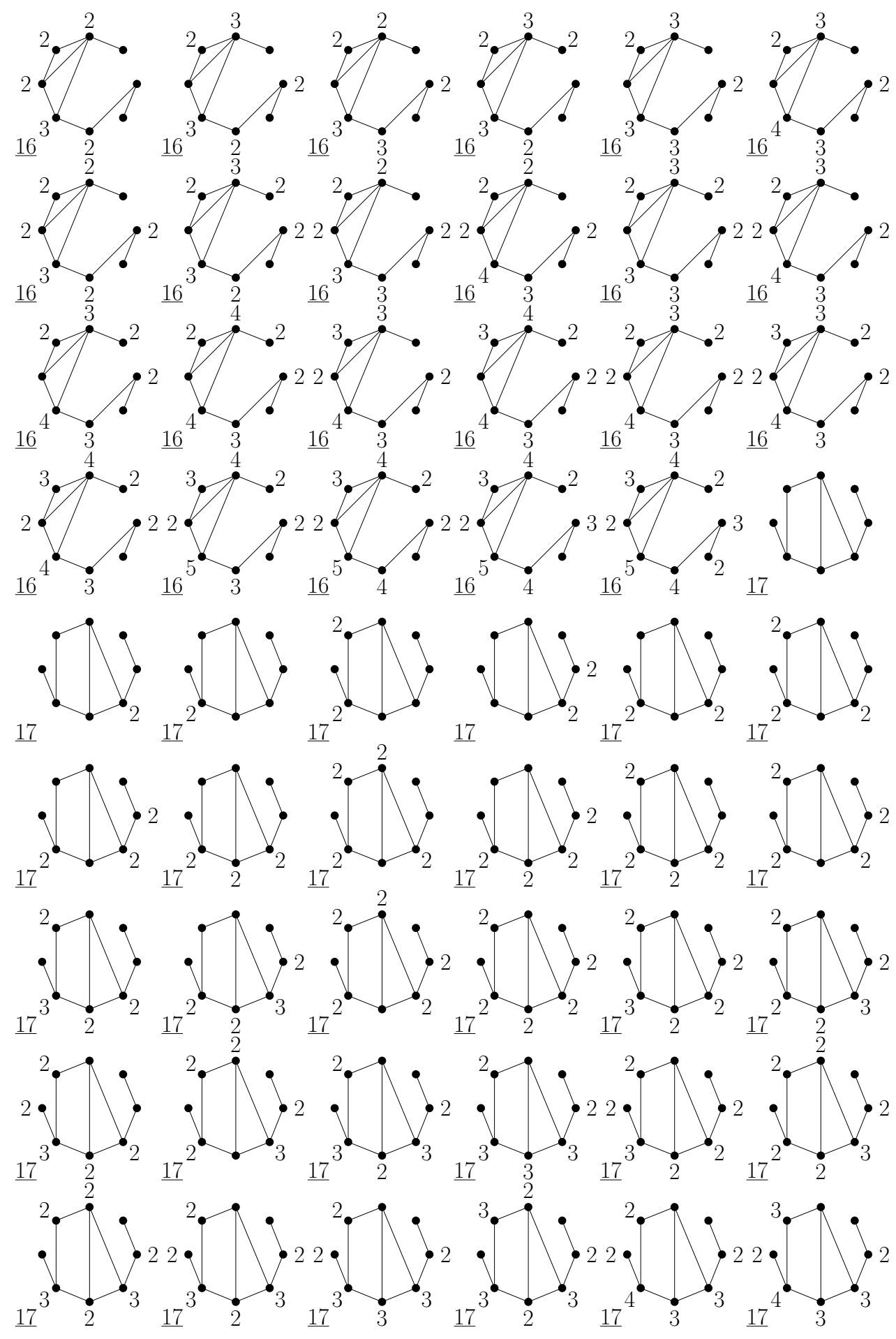

Figure B.25: The set $\mathcal{W}\left(E_{8}\right)$ (continued). 

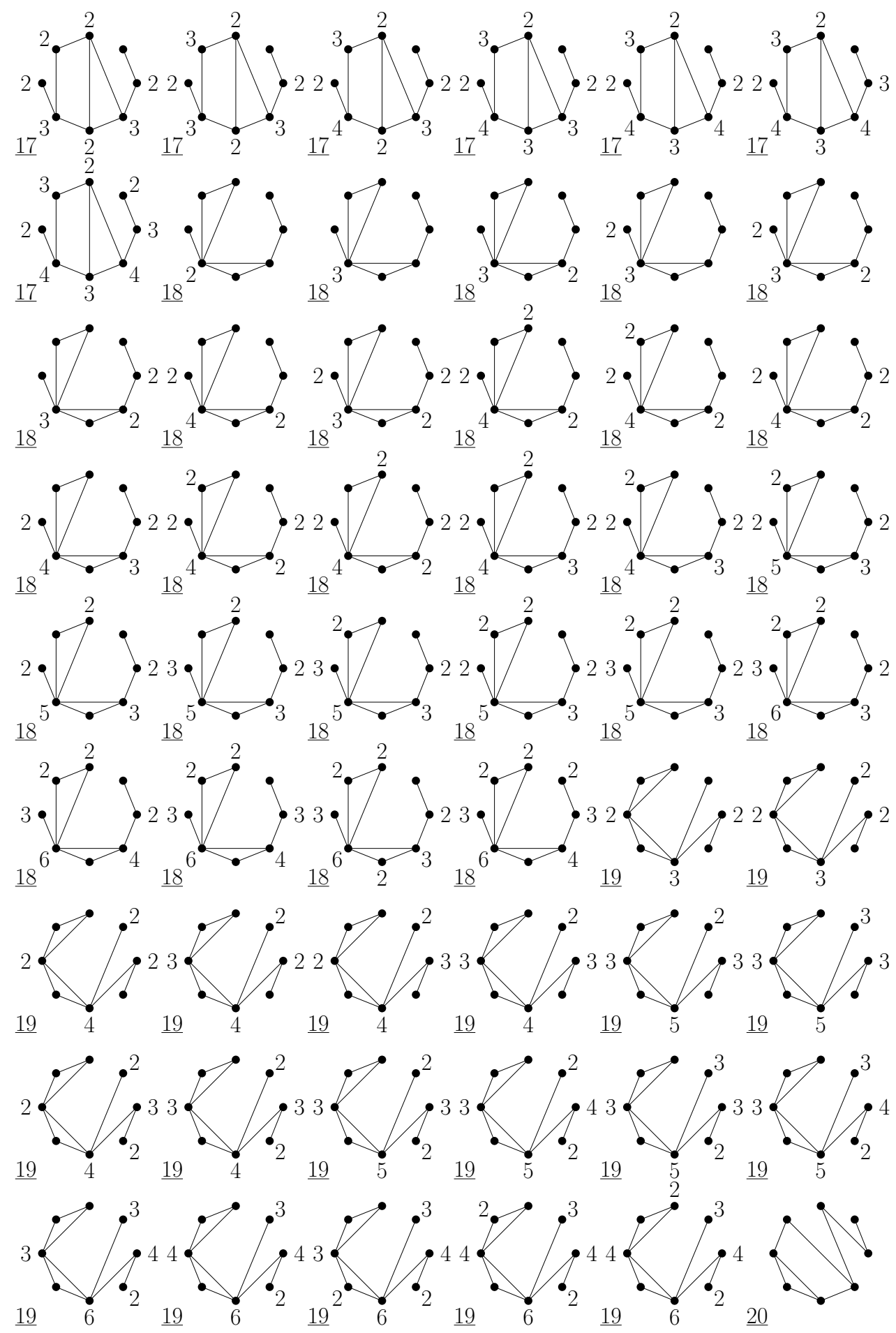

Figure B.26: The set $\mathcal{W}\left(E_{8}\right)$ (continued). 


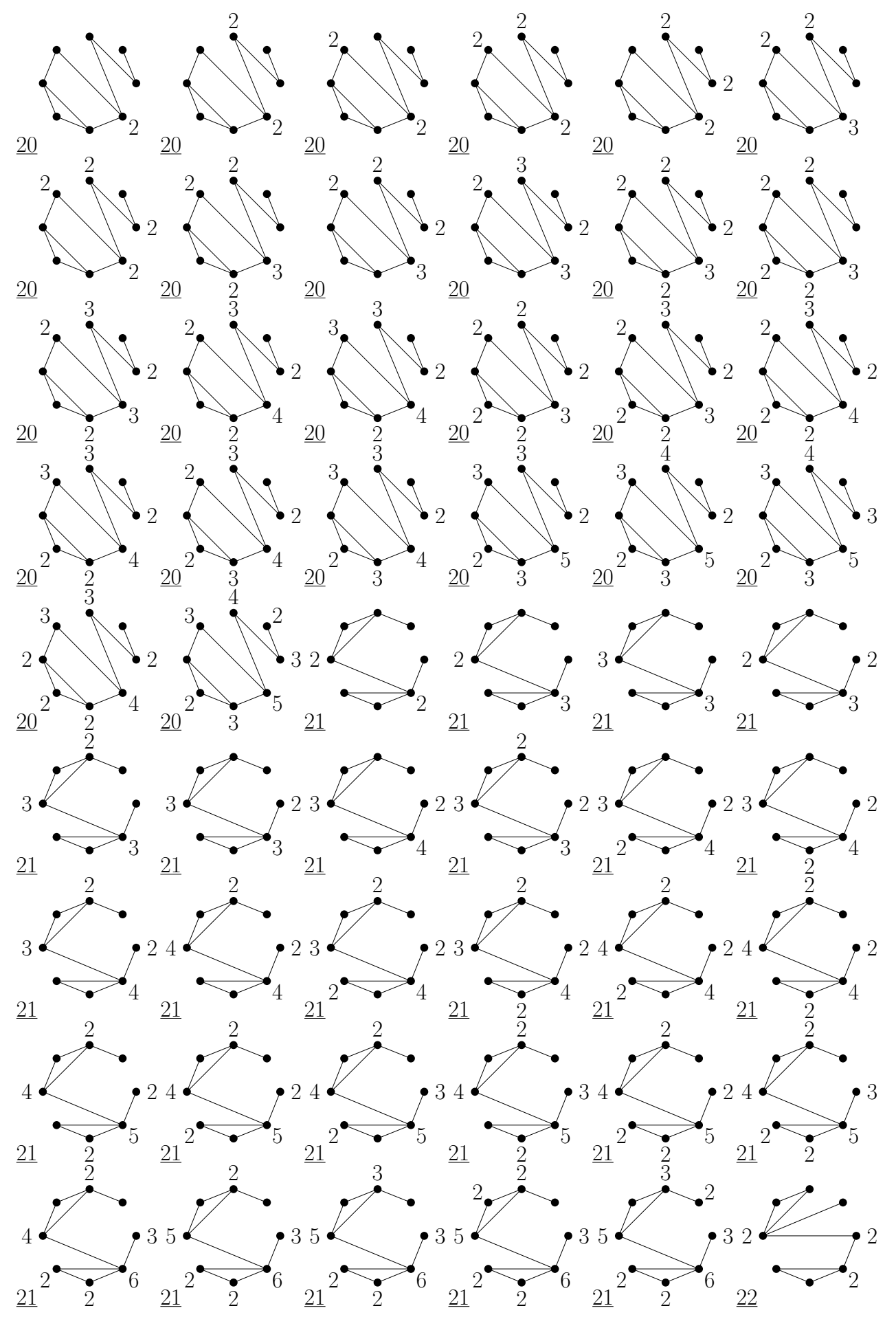

Figure B.27: The set $\mathcal{W}\left(E_{8}\right)$ (continued). 

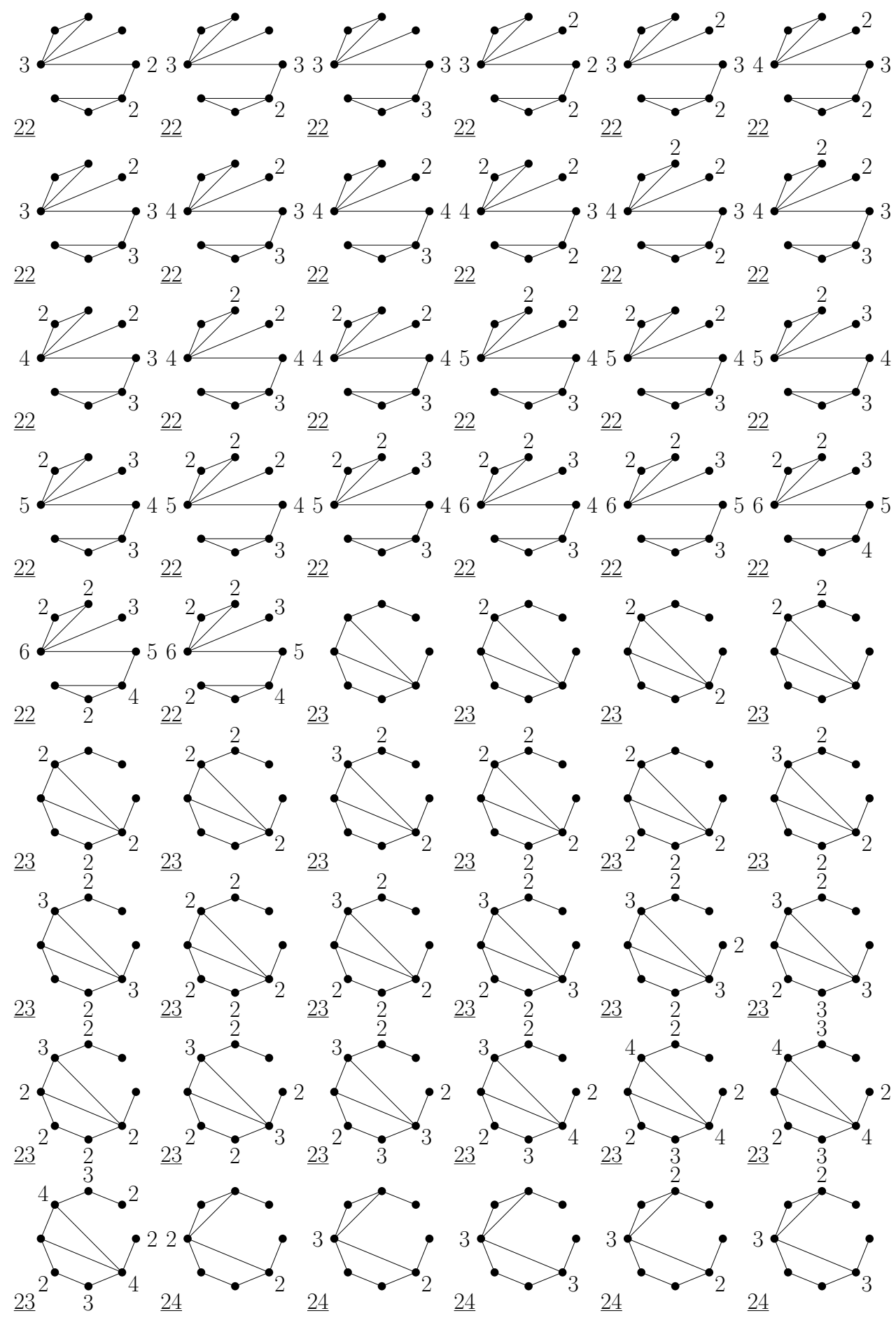

Figure B.28: The set $\mathcal{W}\left(E_{8}\right)$ (continued). 

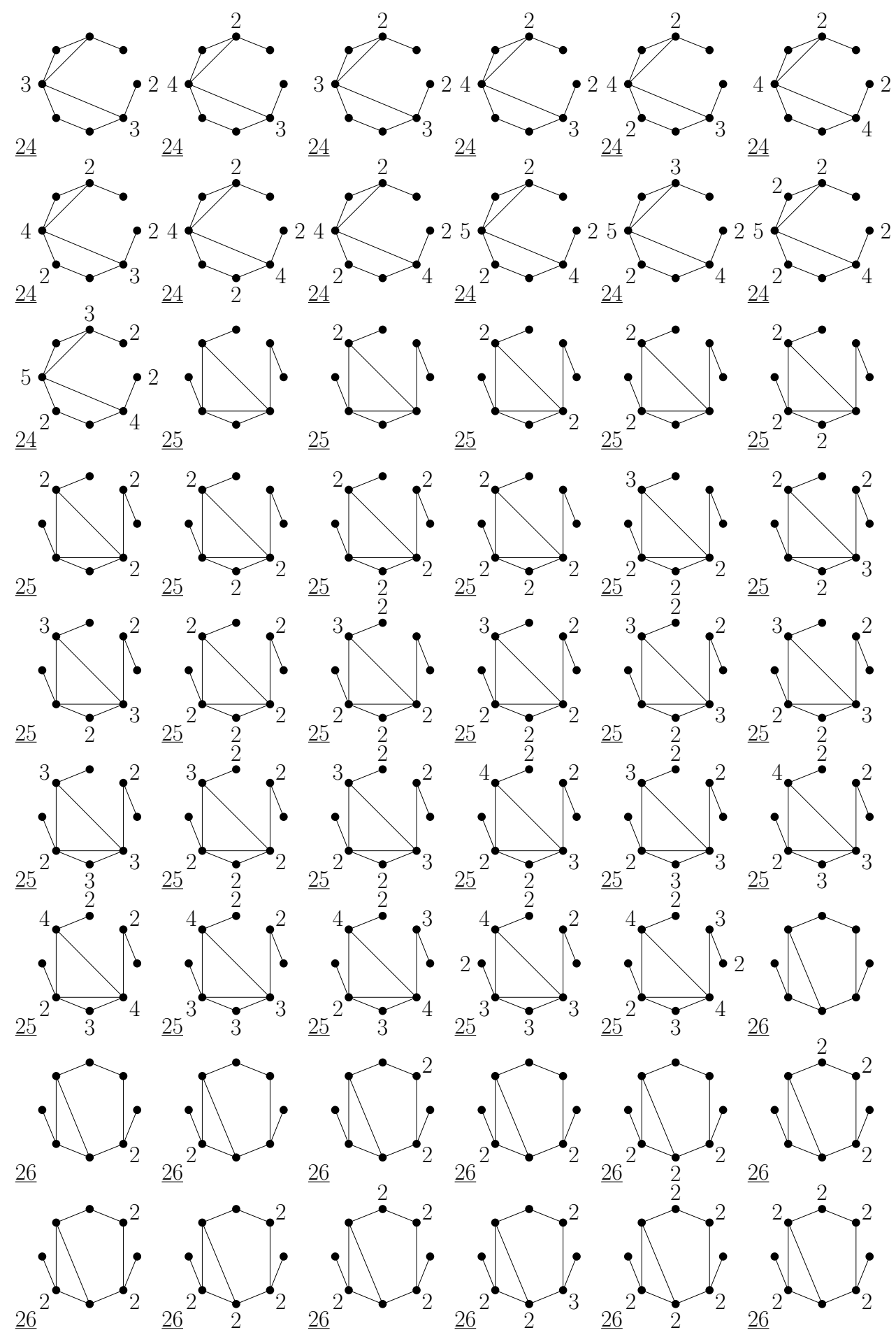

Figure B.29: The set $\mathcal{W}\left(E_{8}\right)$ (continued). 

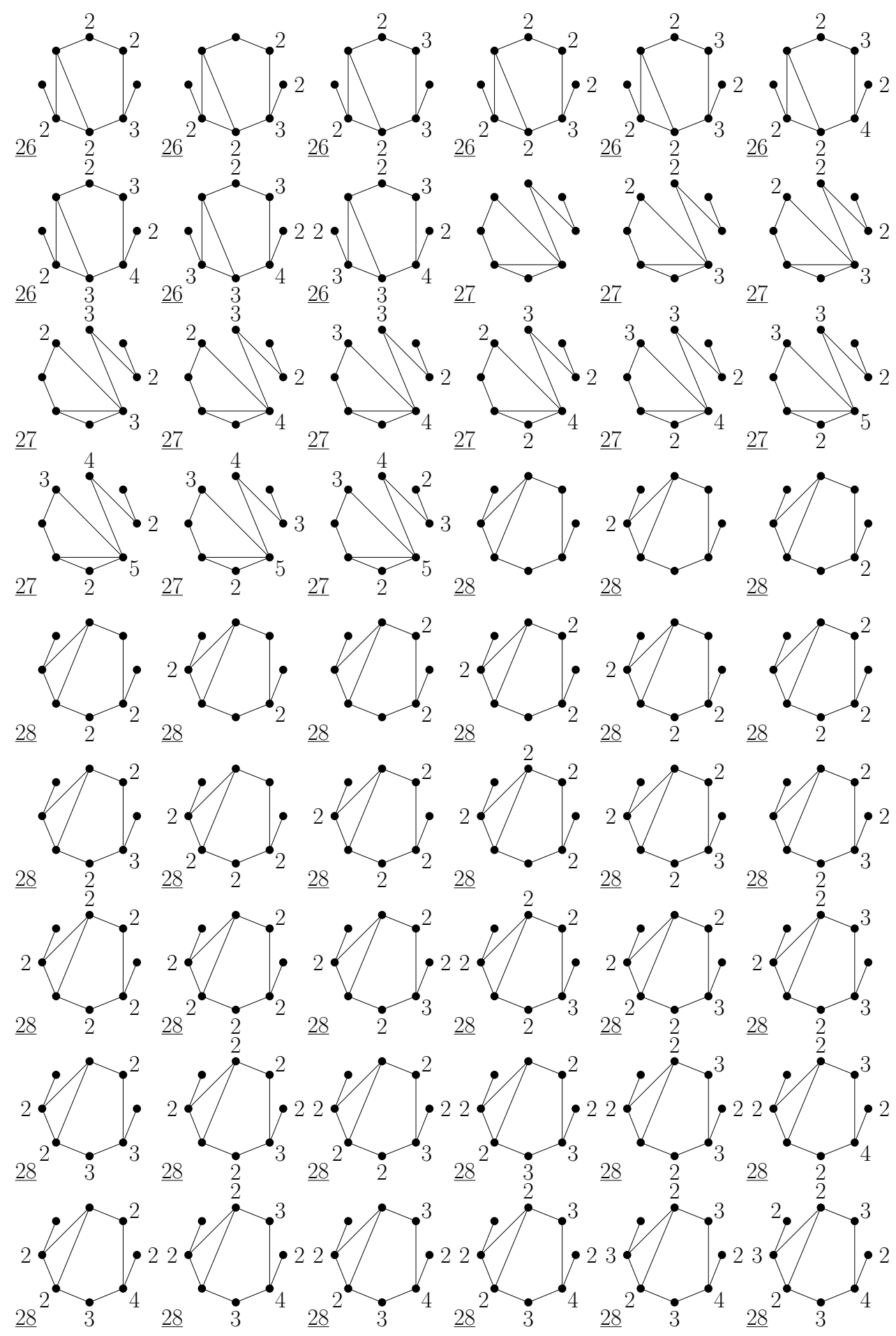

Figure B.30: The set $\mathcal{W}\left(E_{8}\right)$ (continued). 

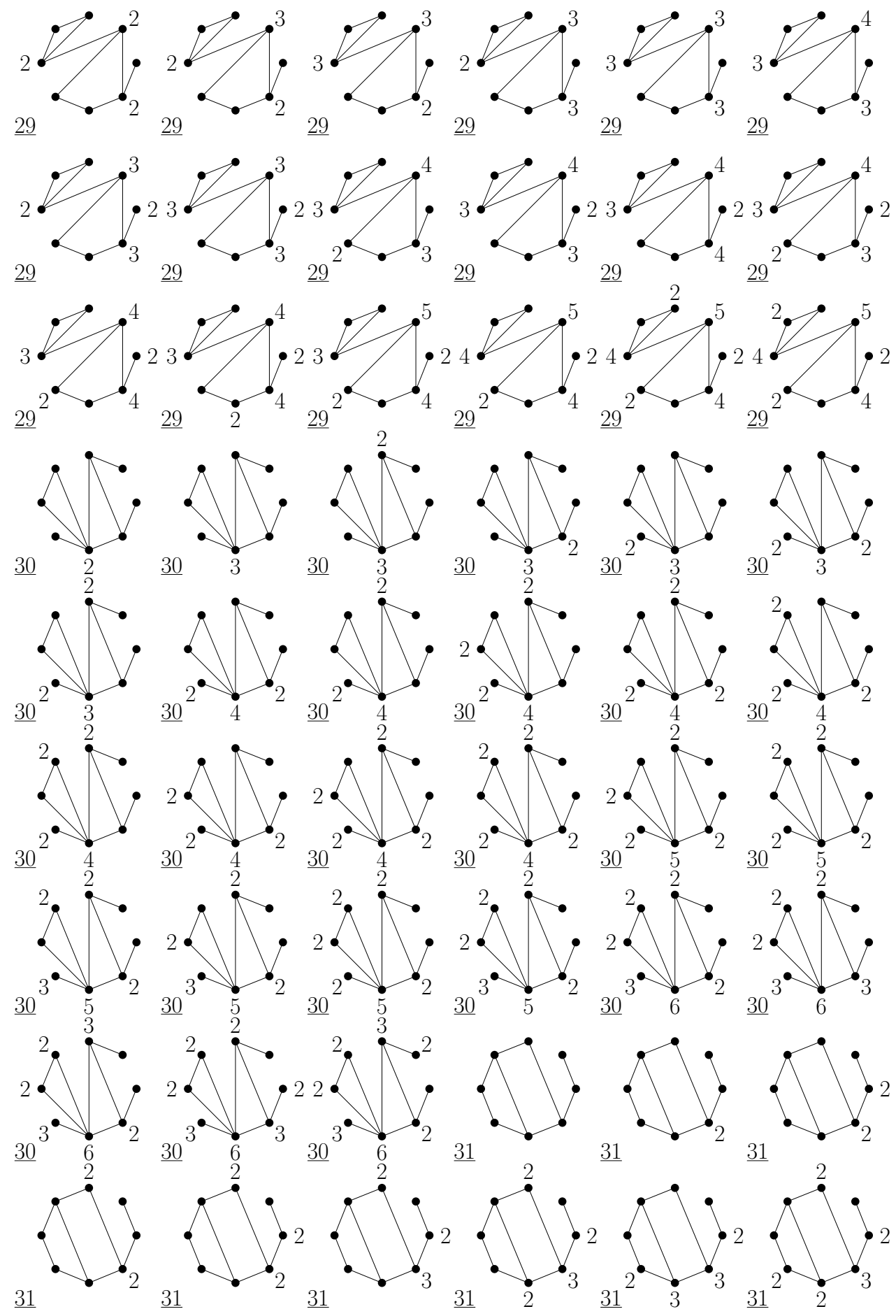

Figure B.31: The set $\mathcal{W}\left(E_{8}\right)$ (continued). 

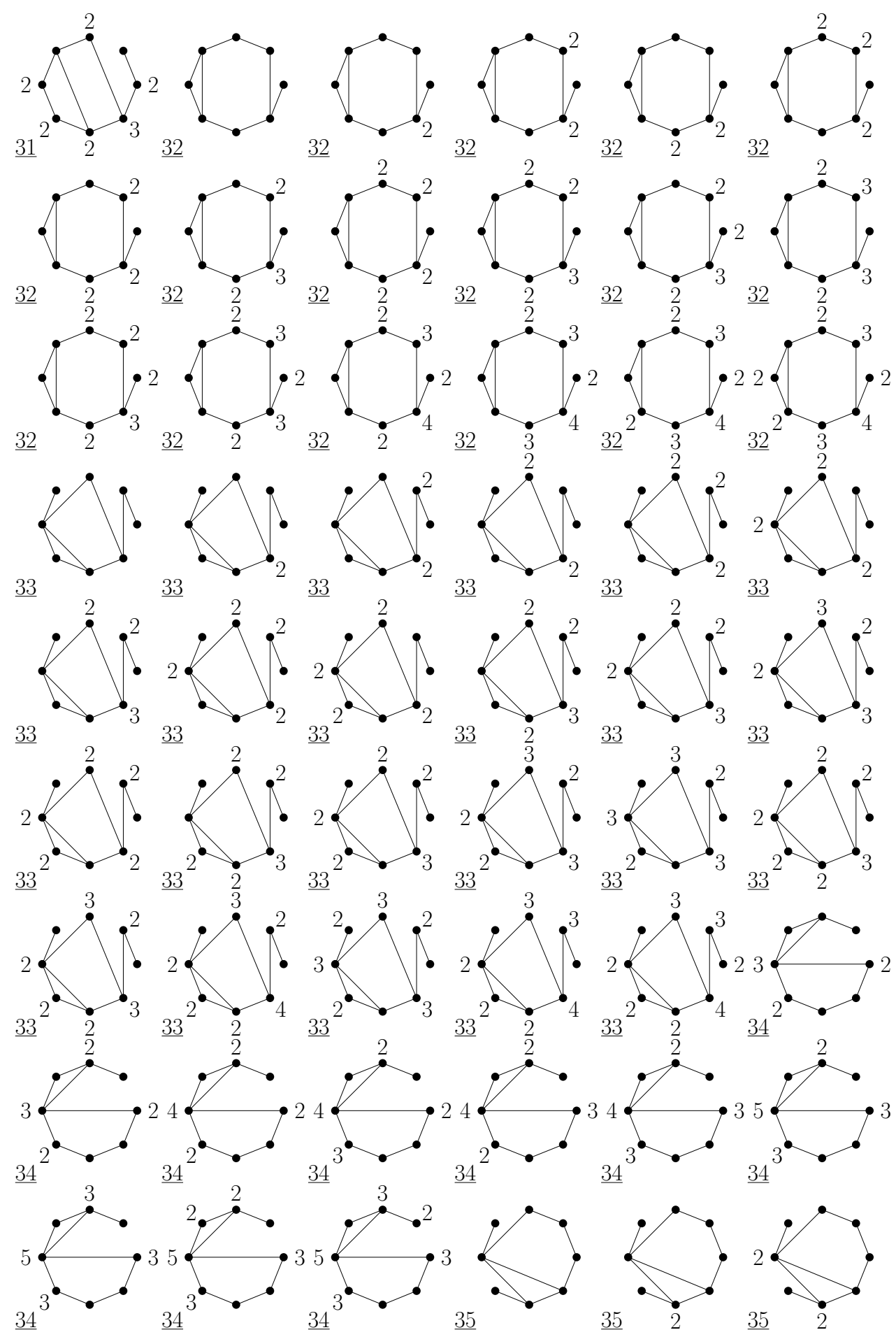

Figure B.32: The set $\mathcal{W}\left(E_{8}\right)$ (continued). 


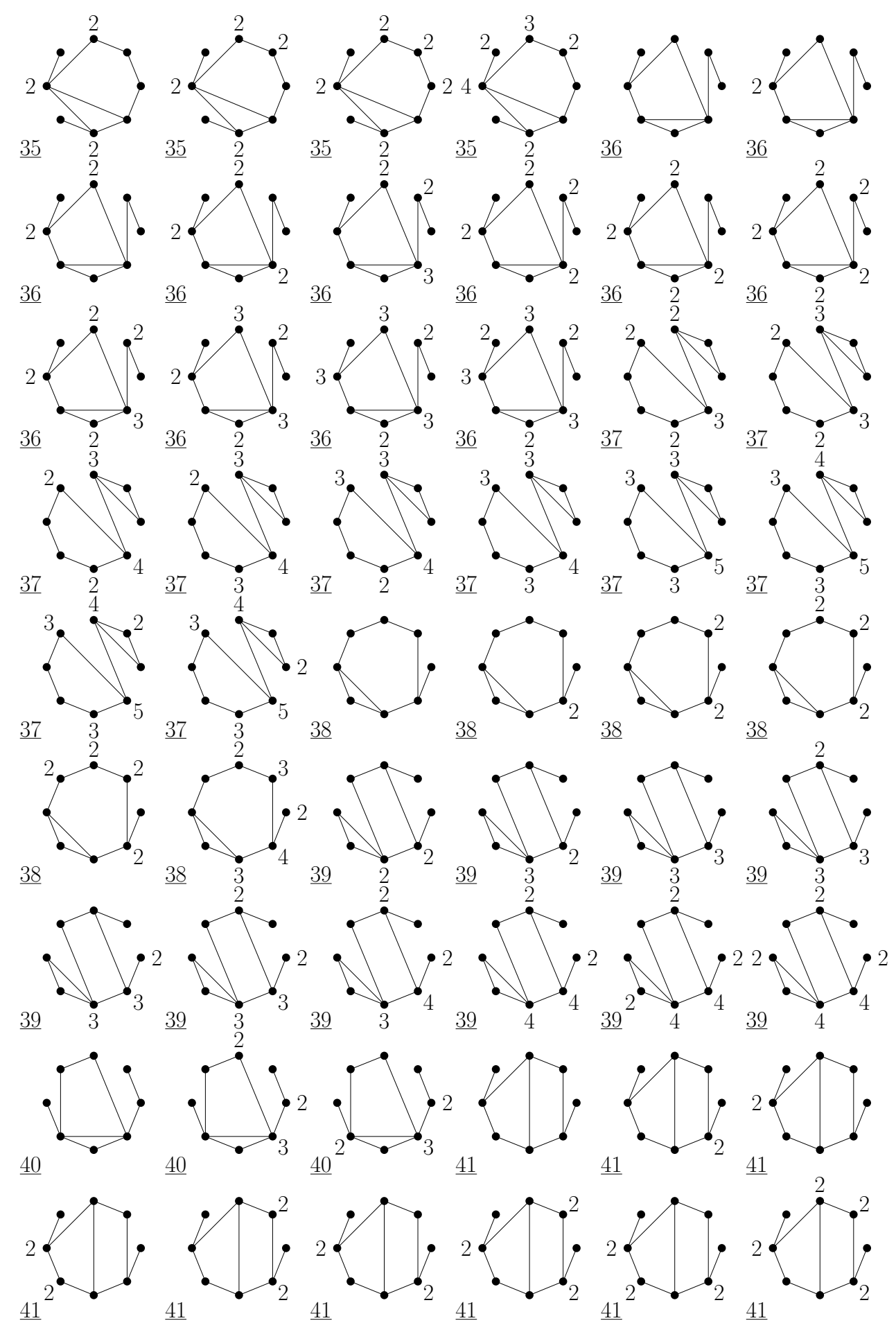

Figure B.33: The set $\mathcal{W}\left(E_{8}\right)$ (continued). 

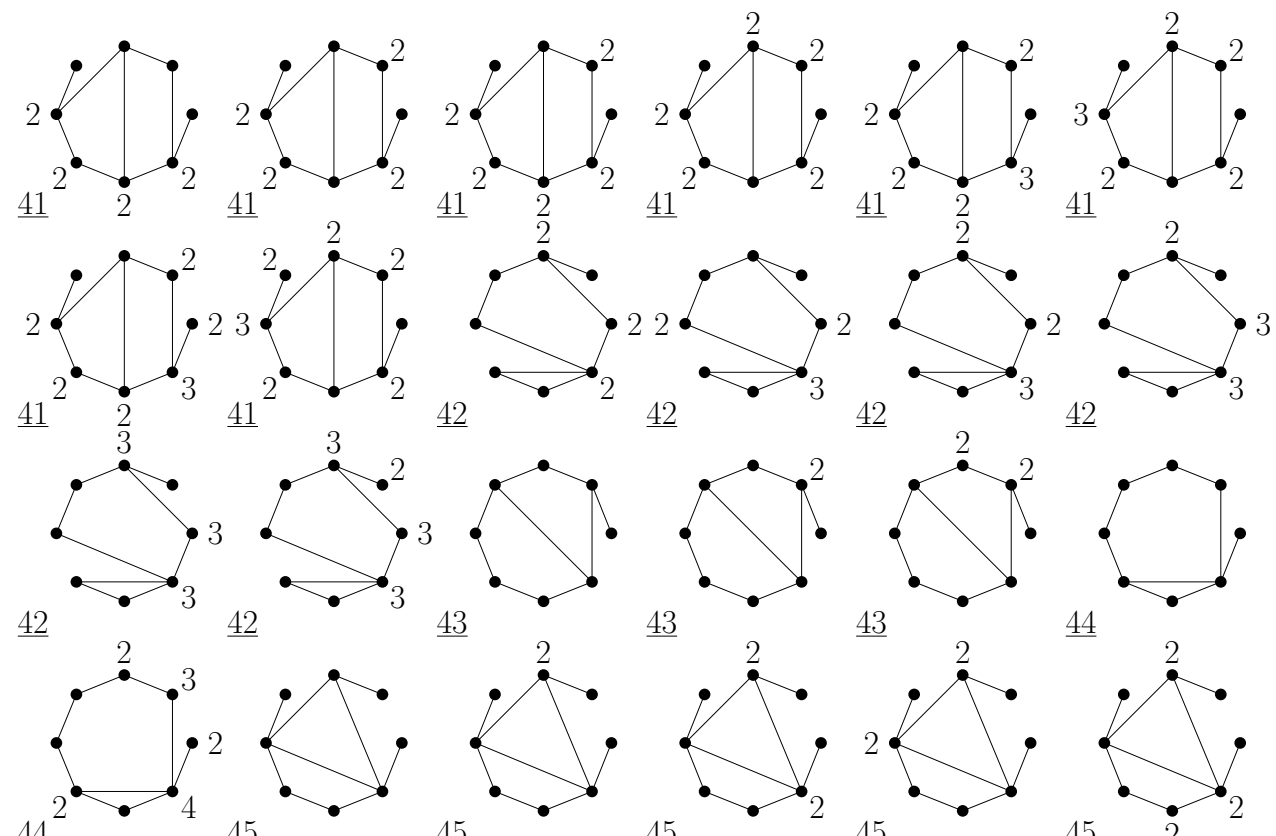

$\underline{43}$
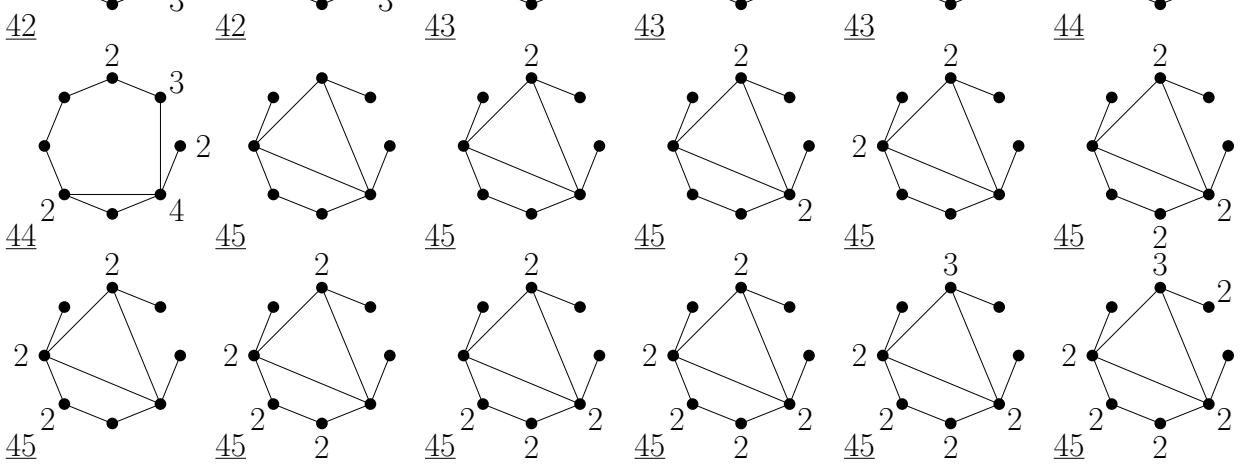

$45-45$

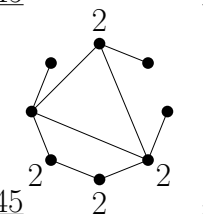

45

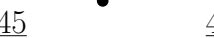

年
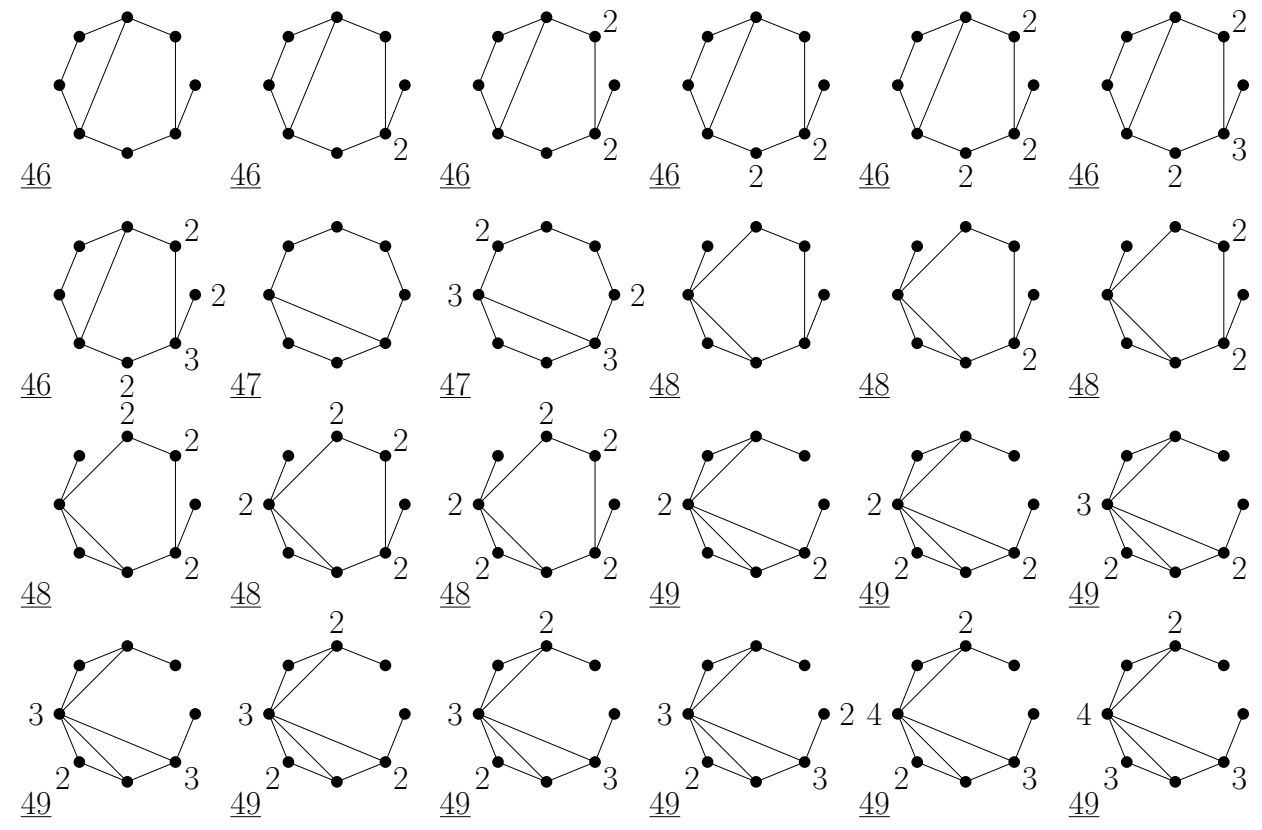

Figure B.34: The set $\mathcal{W}\left(E_{8}\right)$ (continued). 

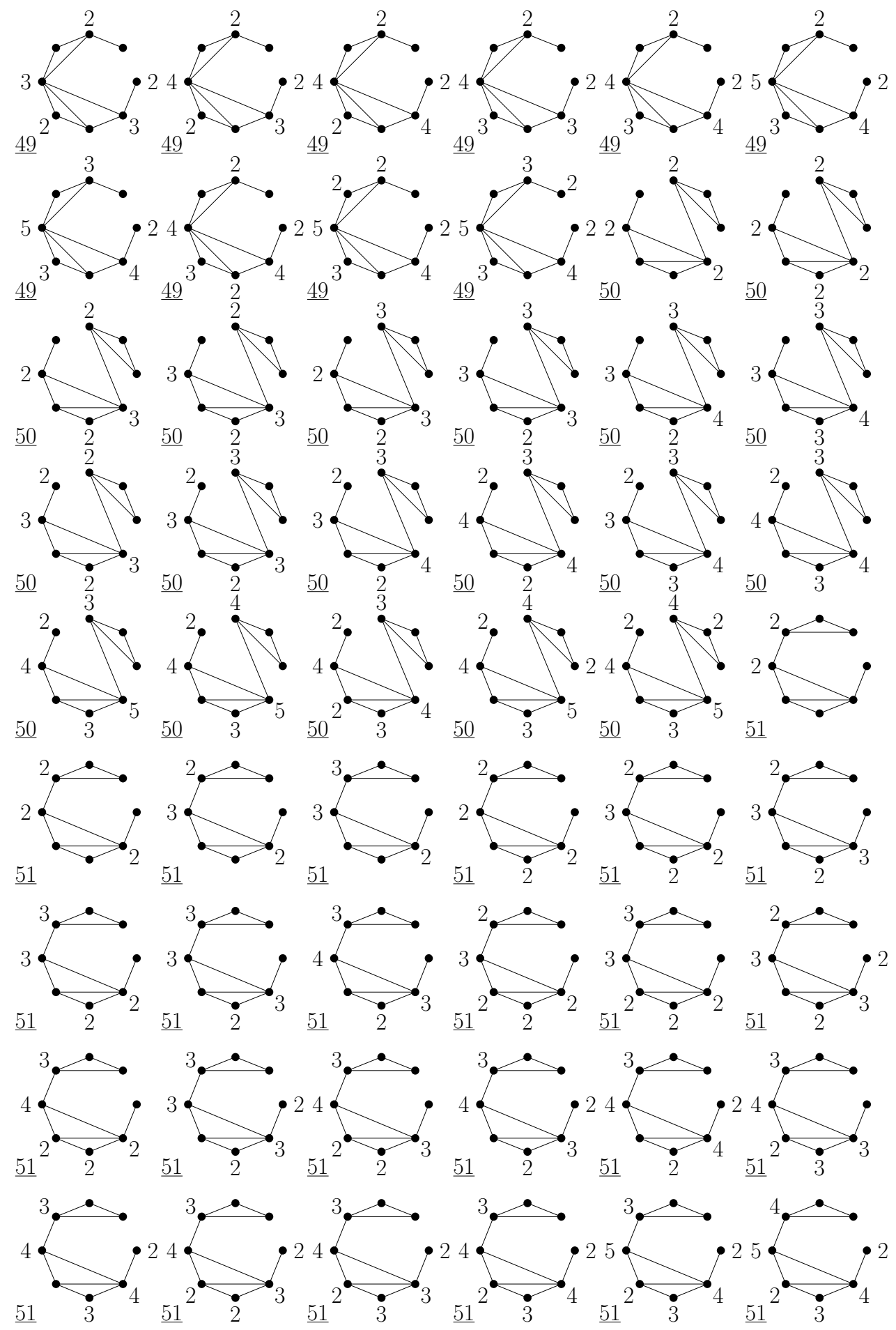

Figure B.35: The set $\mathcal{W}\left(E_{8}\right)$ (continued). 


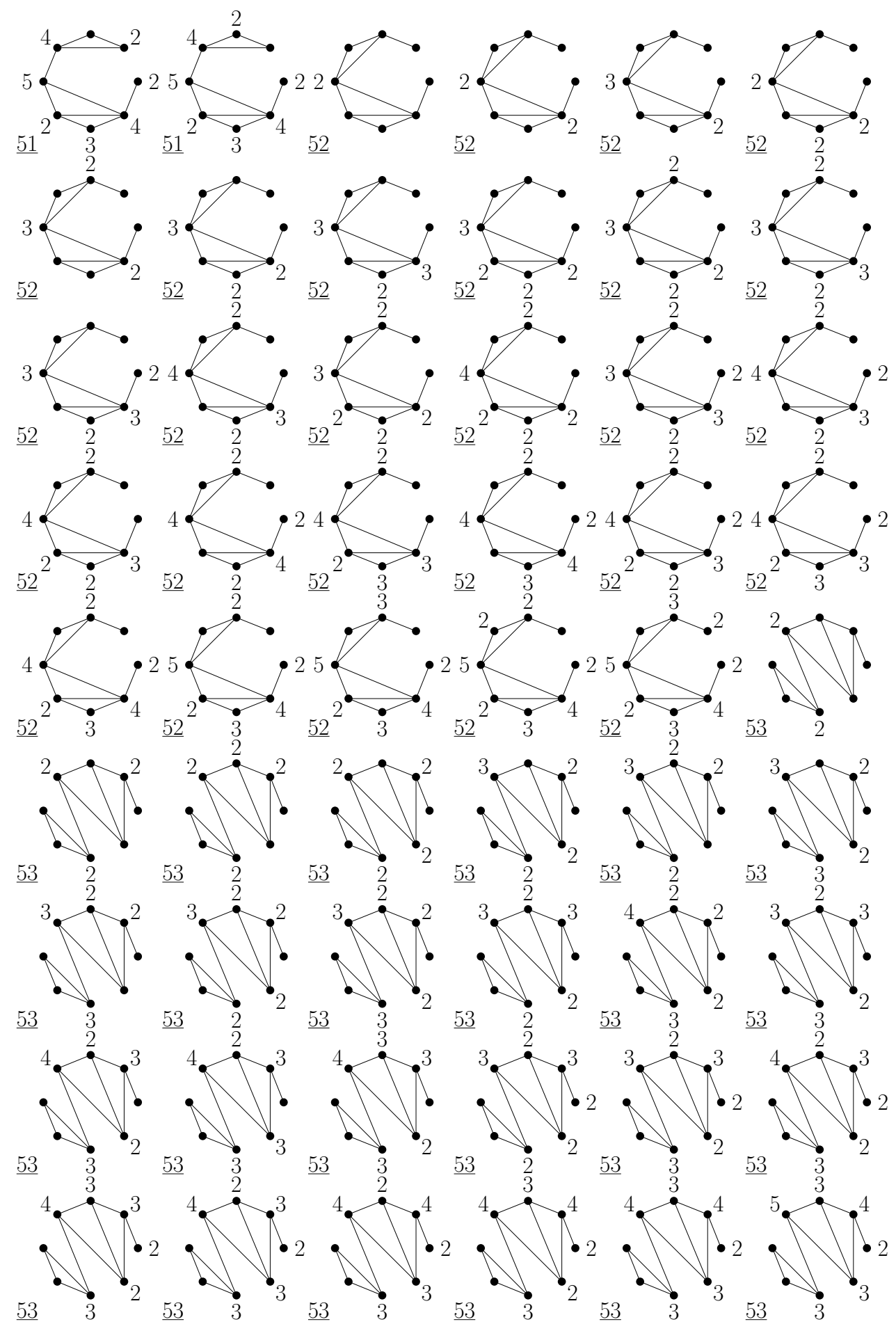

Figure B.36: The set $\mathcal{W}\left(E_{8}\right)$ (continued). 


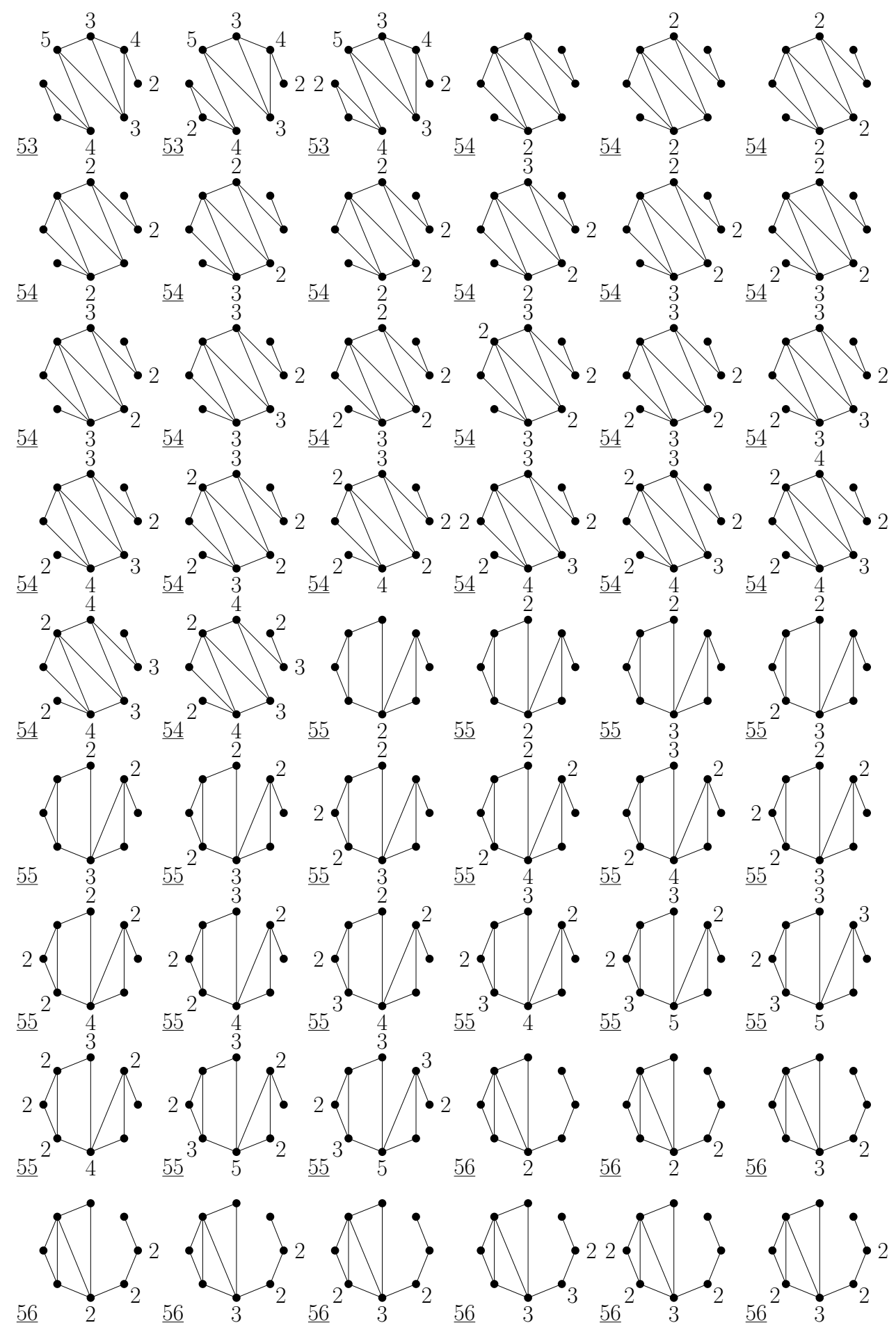

Figure B.37: The set $\mathcal{W}\left(E_{8}\right)$ (continued). 

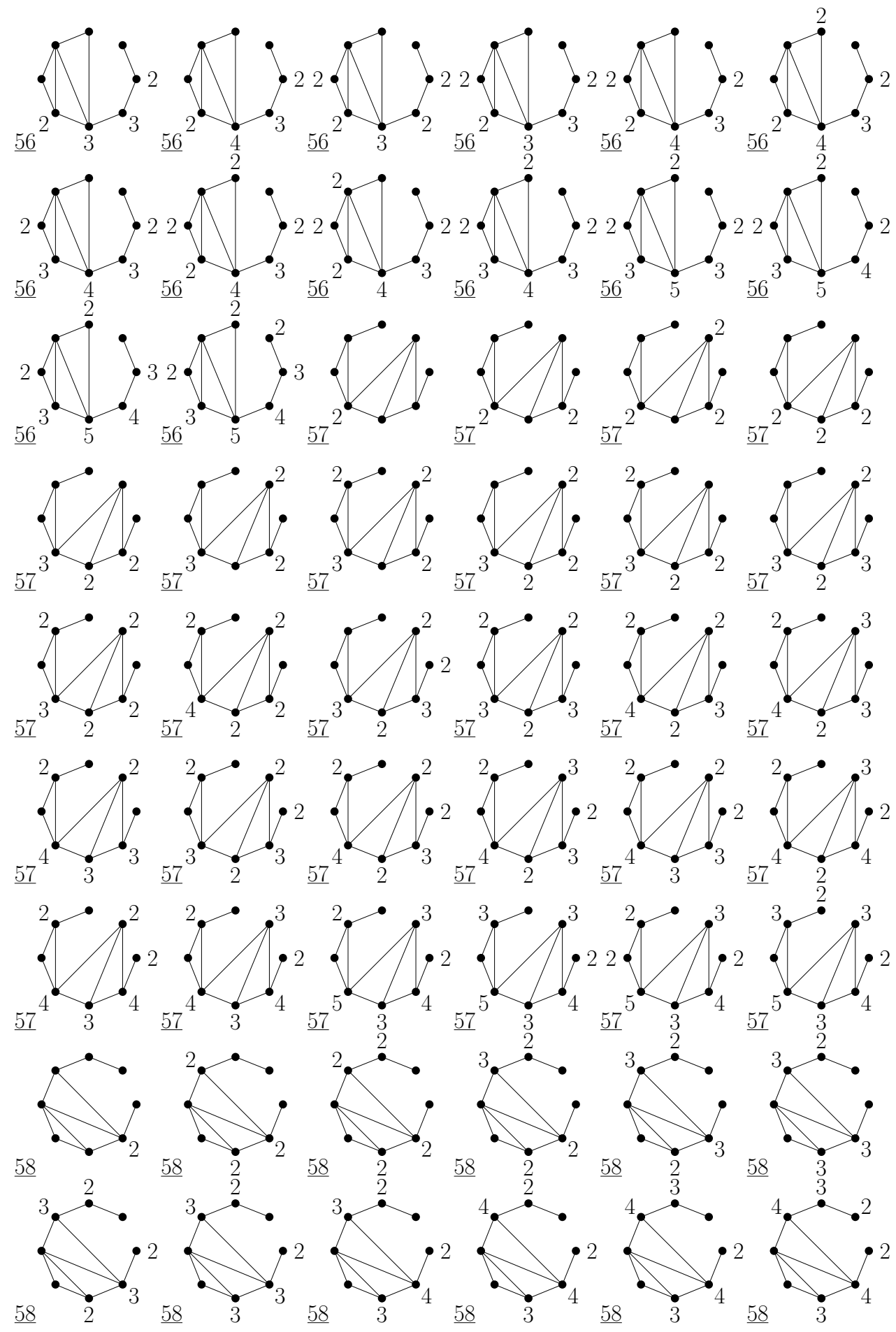

Figure B.38: The set $\mathcal{W}\left(E_{8}\right)$ (continued). 


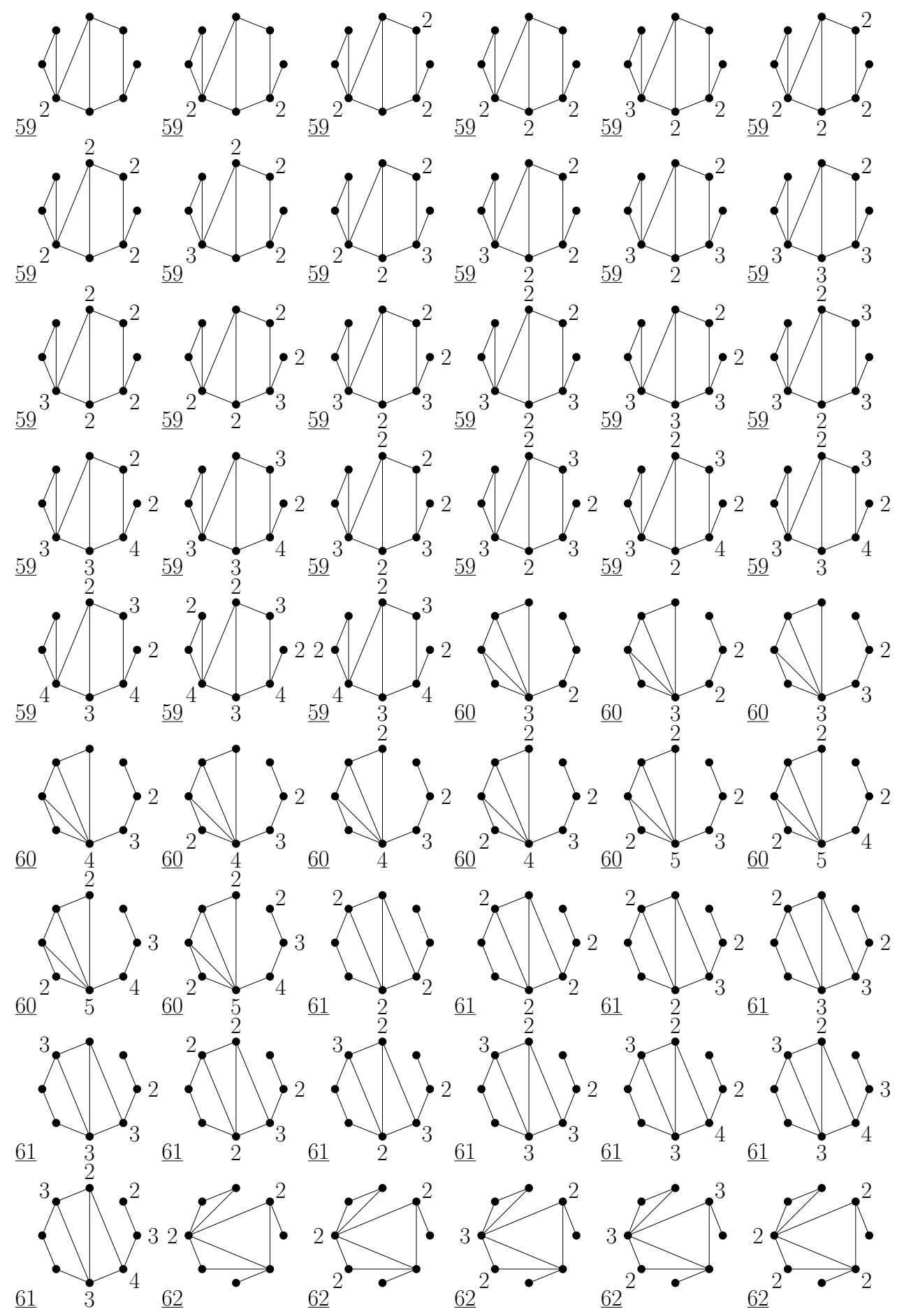

Figure B.39: The set $\mathcal{W}\left(E_{8}\right)$ (continued). 

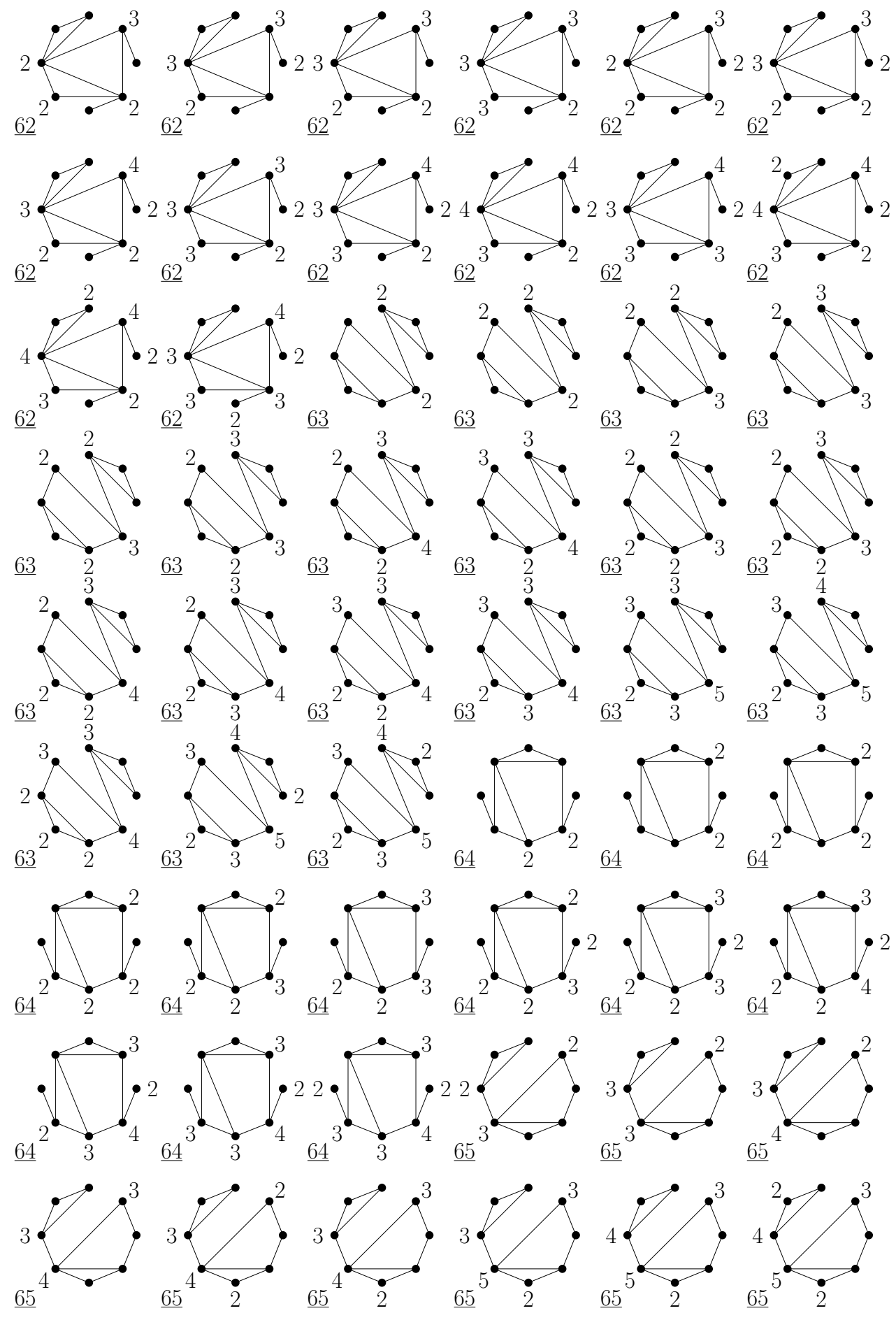

Figure B.40: The set $\mathcal{W}\left(E_{8}\right)$ (continued). 

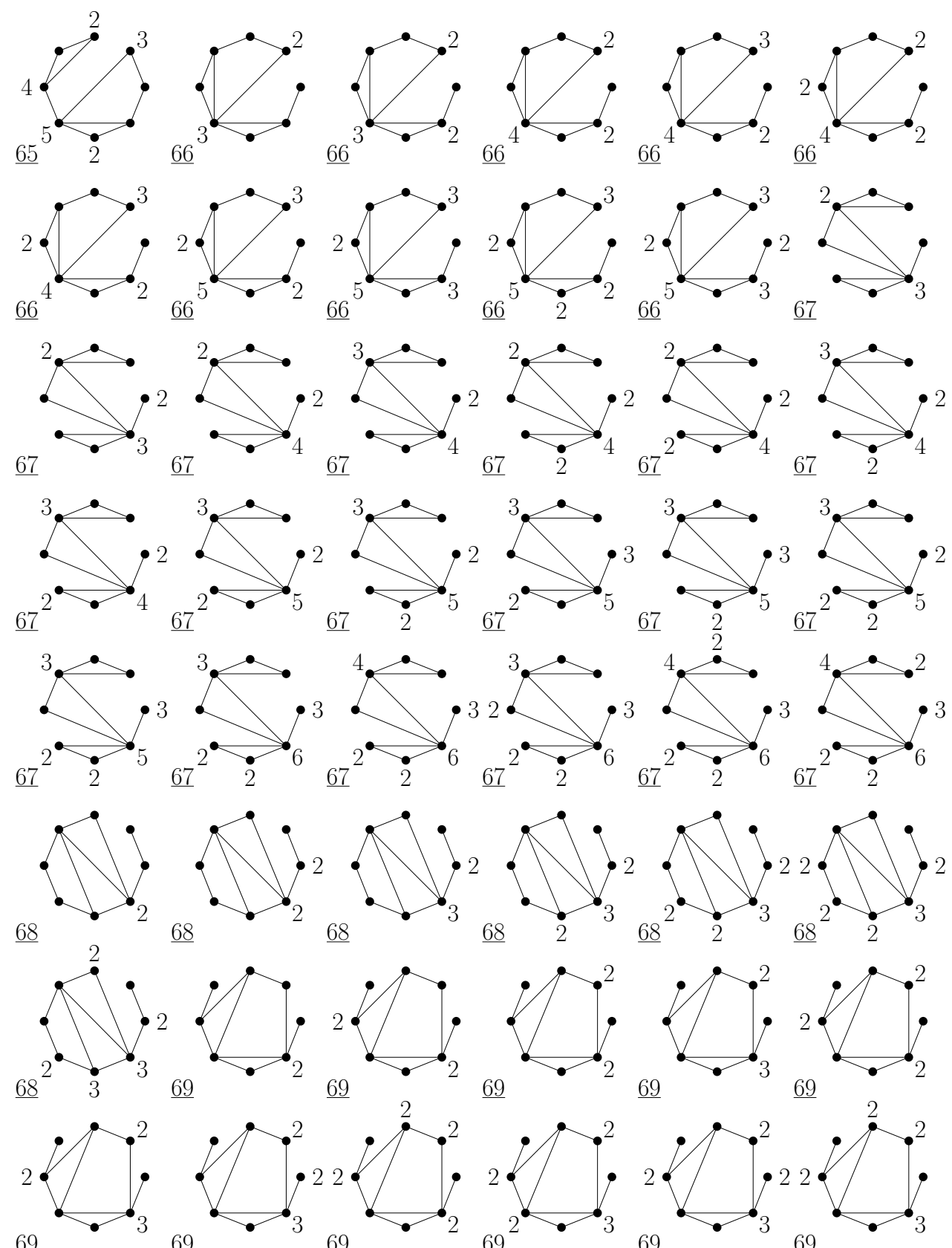

$\underline{69}$
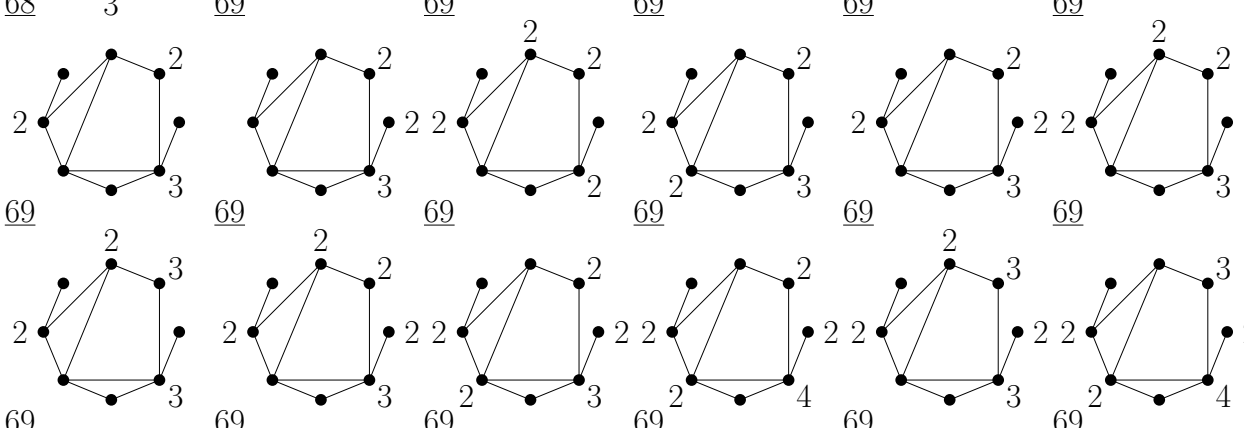

$\underline{69}$
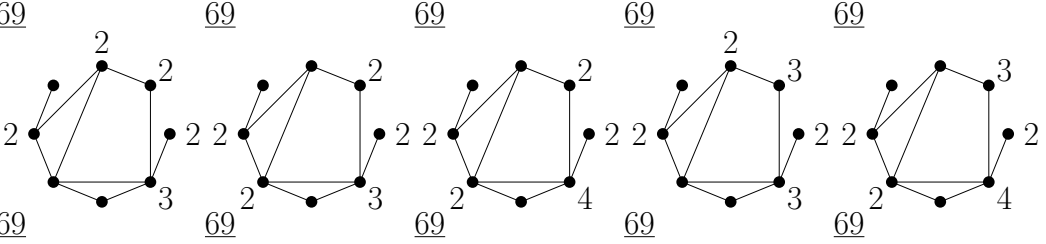

Figure B.41: The set $\mathcal{W}\left(E_{8}\right)$ (continued). 

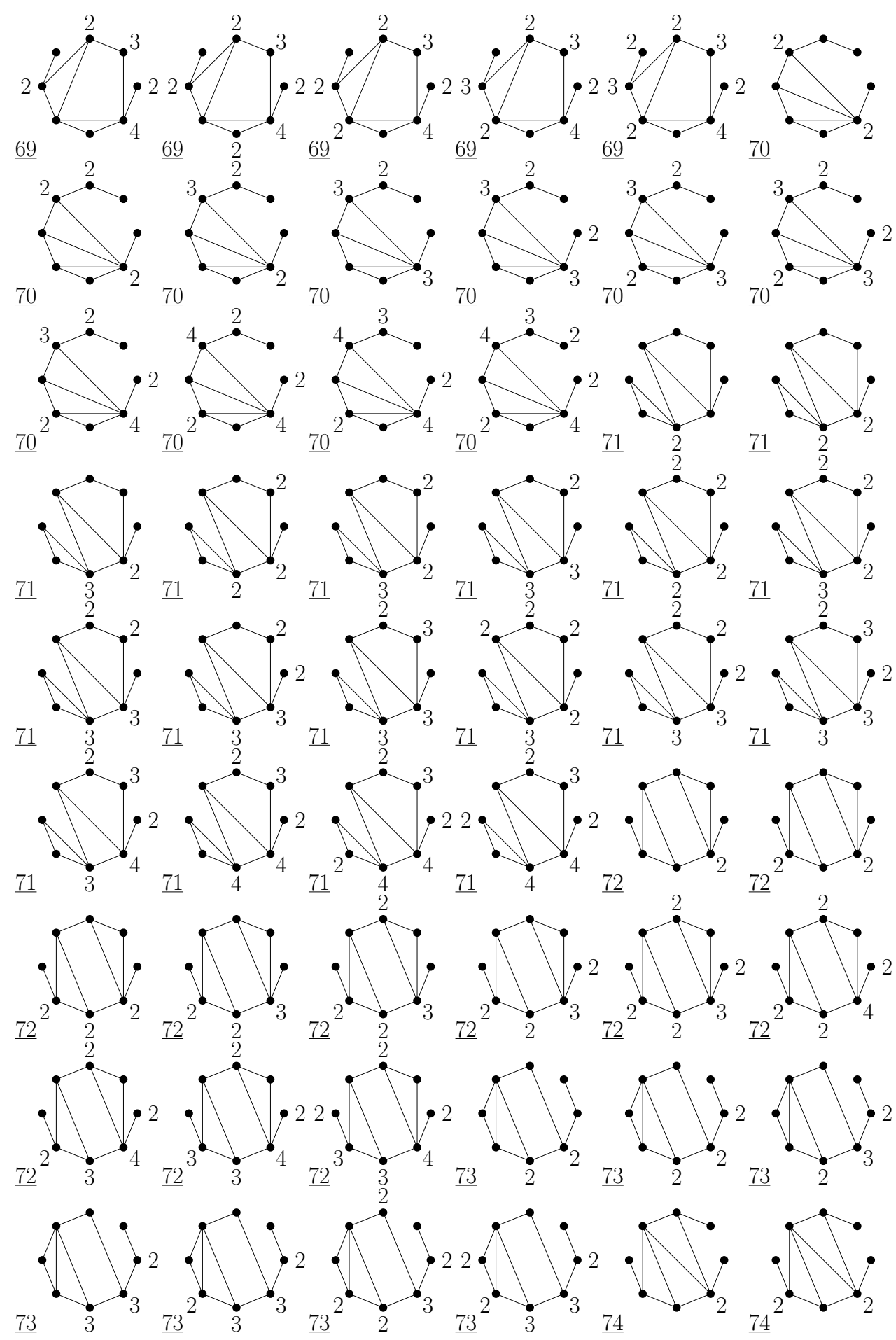

Figure B.42: The set $\mathcal{W}\left(E_{8}\right)$ (continued). 

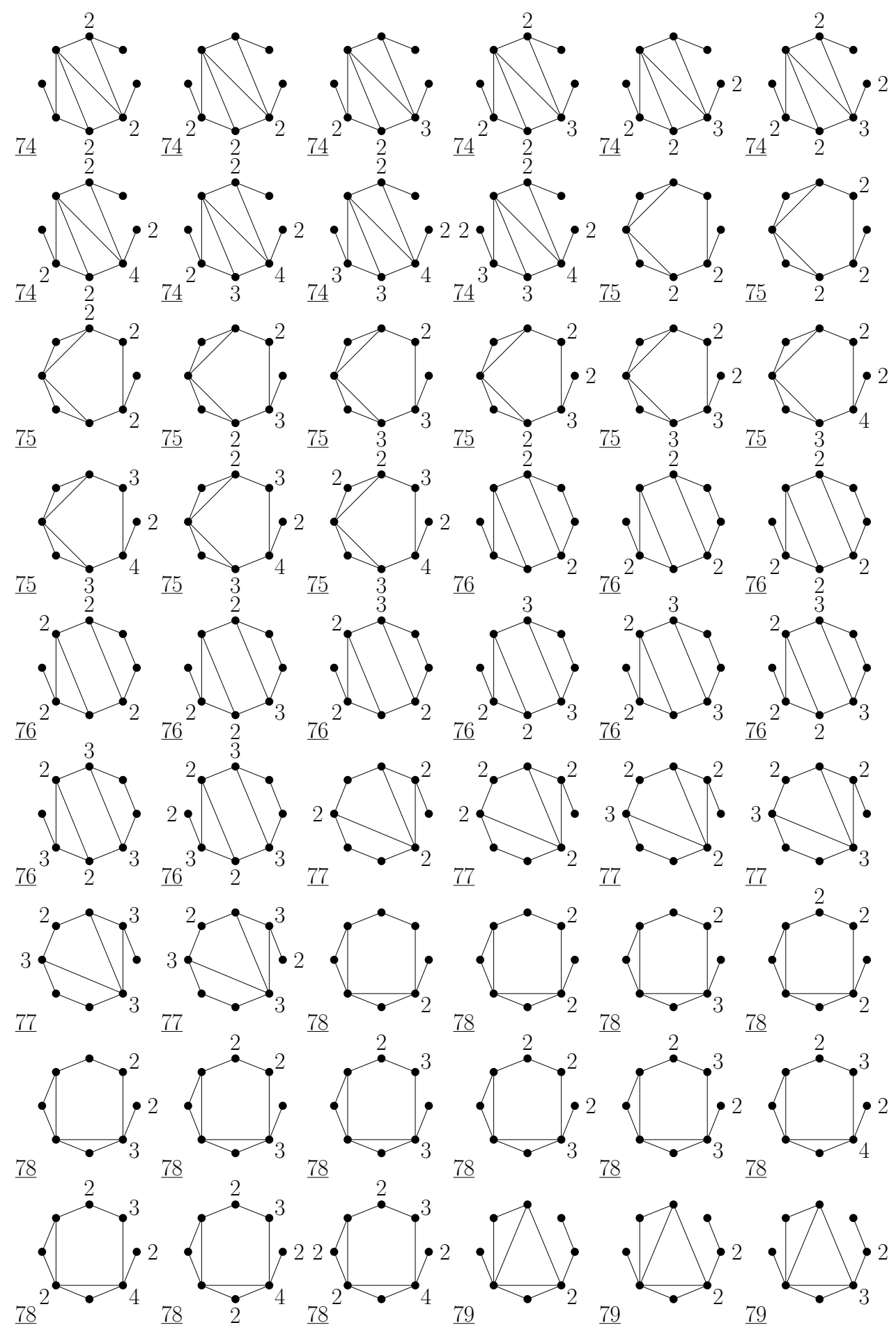

Figure B.43: The set $\mathcal{W}\left(E_{8}\right)$ (continued). 

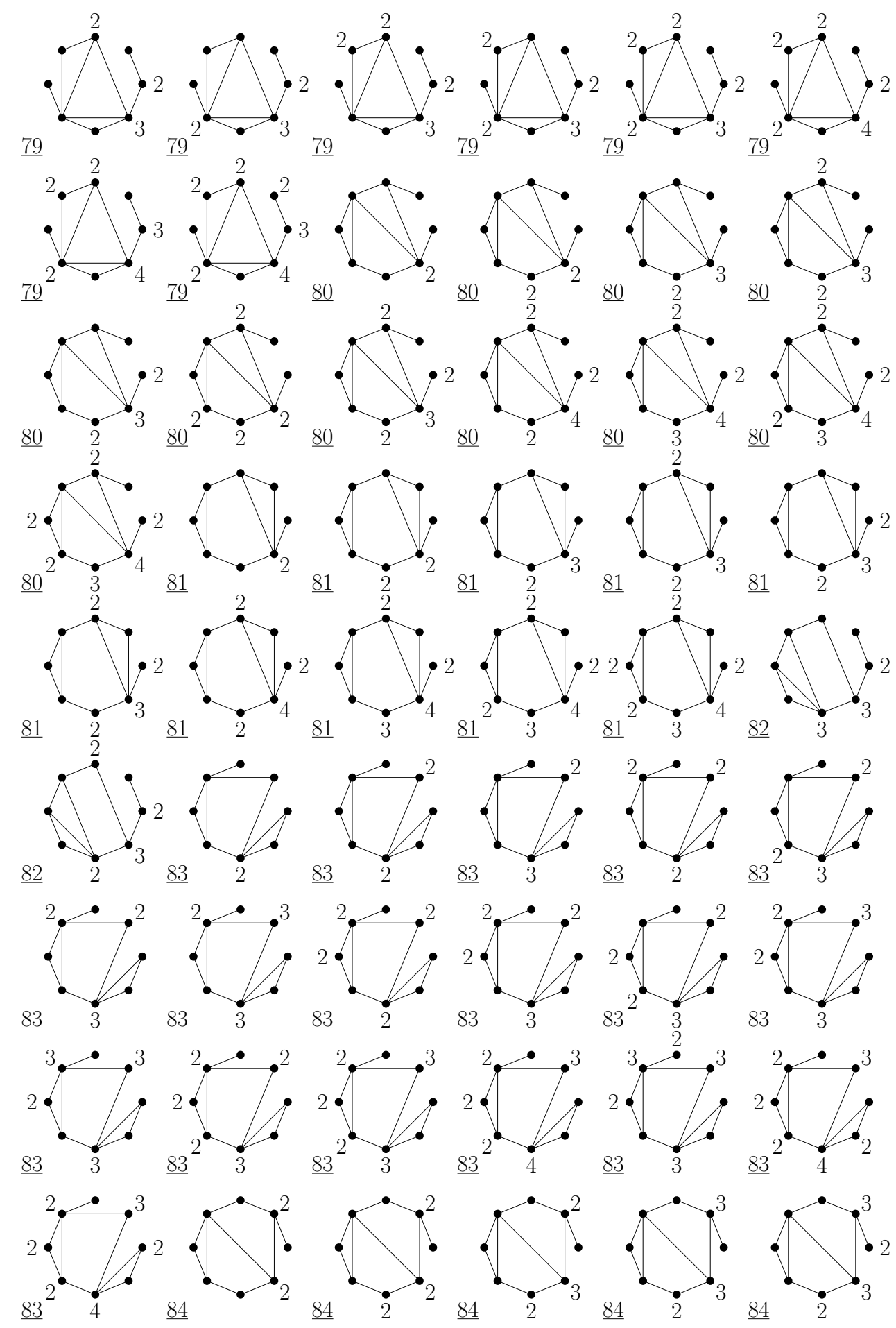

Figure B.44: The set $\mathcal{W}\left(E_{8}\right)$ (continued). 

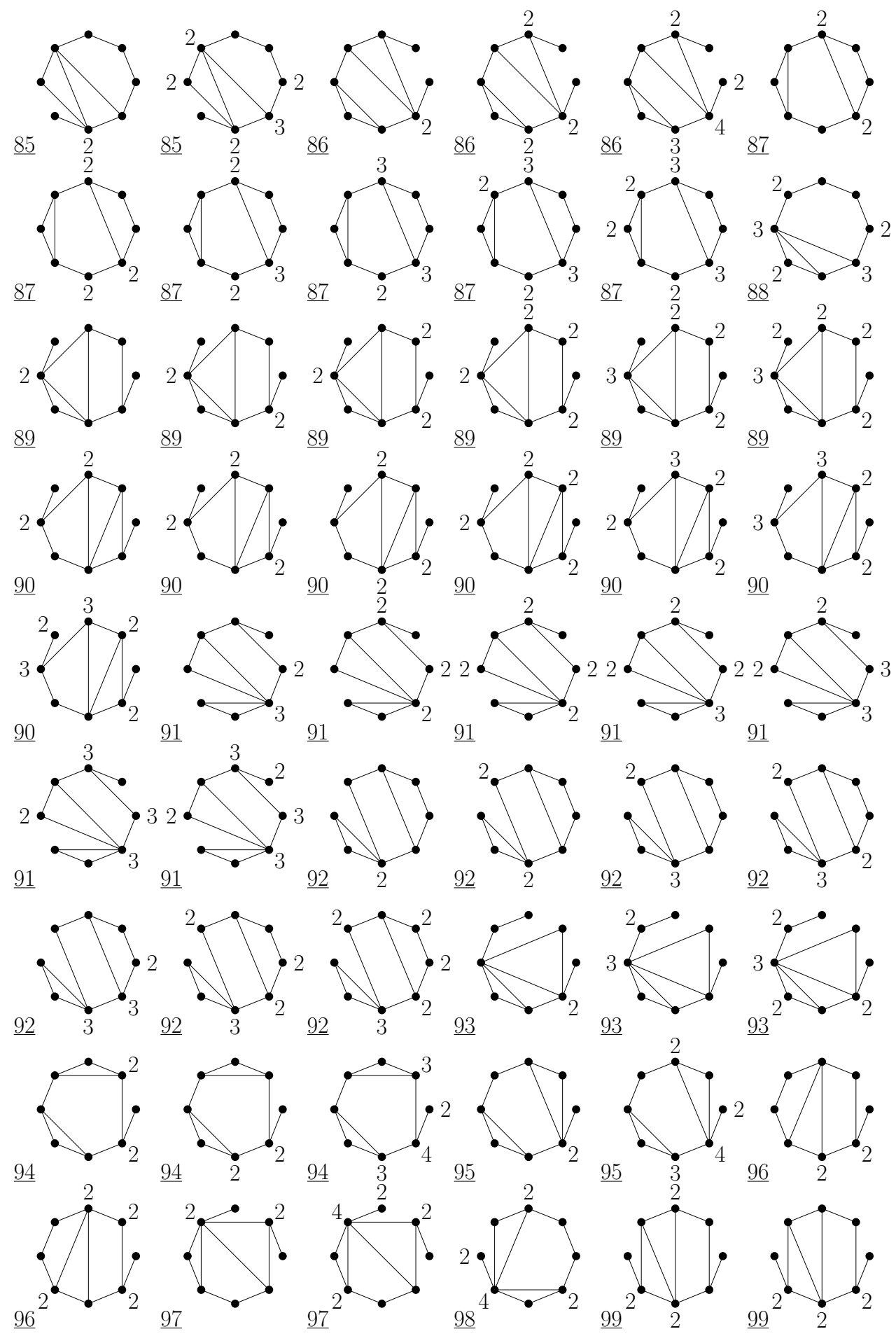

Figure B.45: The set $\mathcal{W}\left(E_{8}\right)$ (continued). 

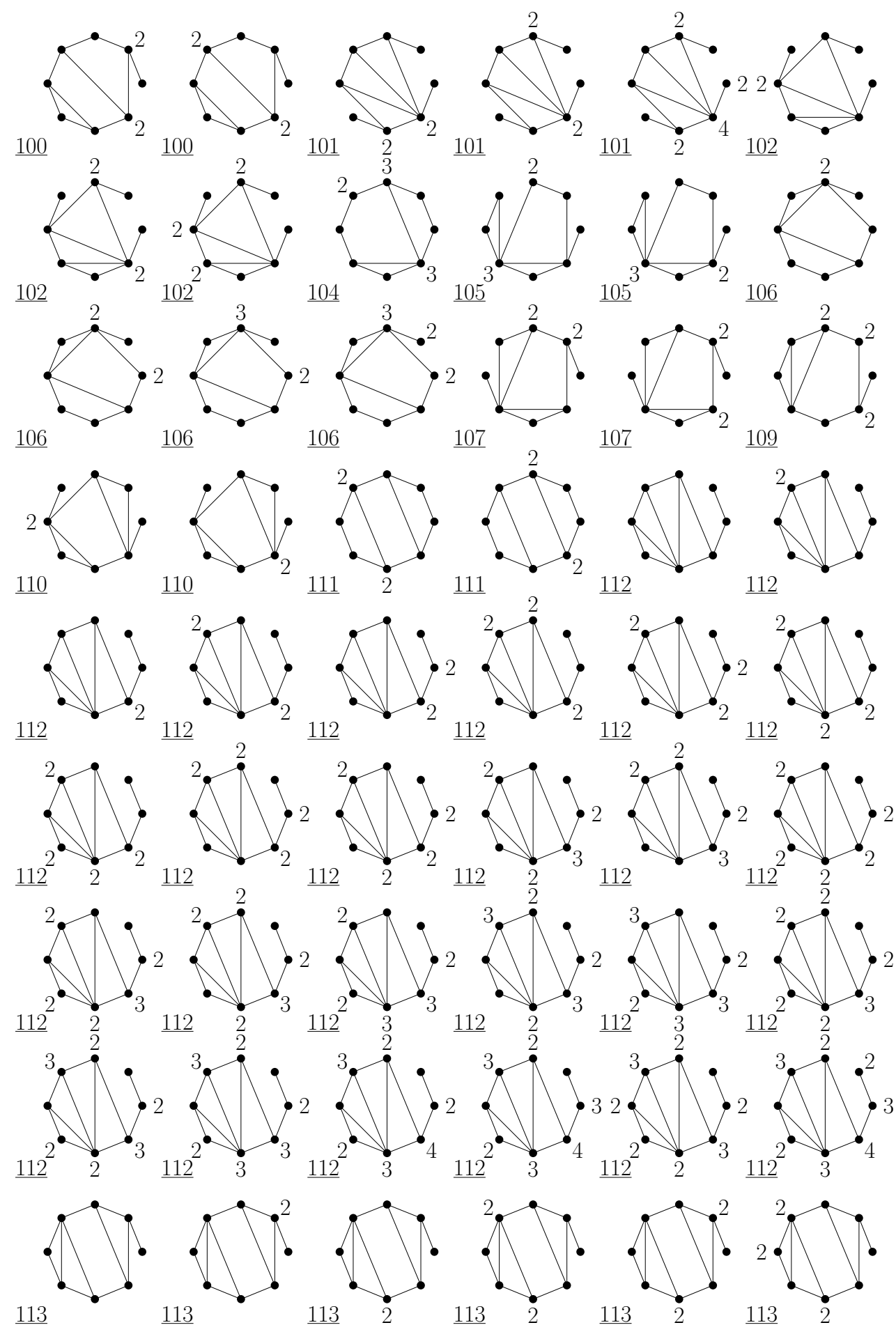

Figure B.46: The set $\mathcal{W}\left(E_{8}\right)$ (continued). 

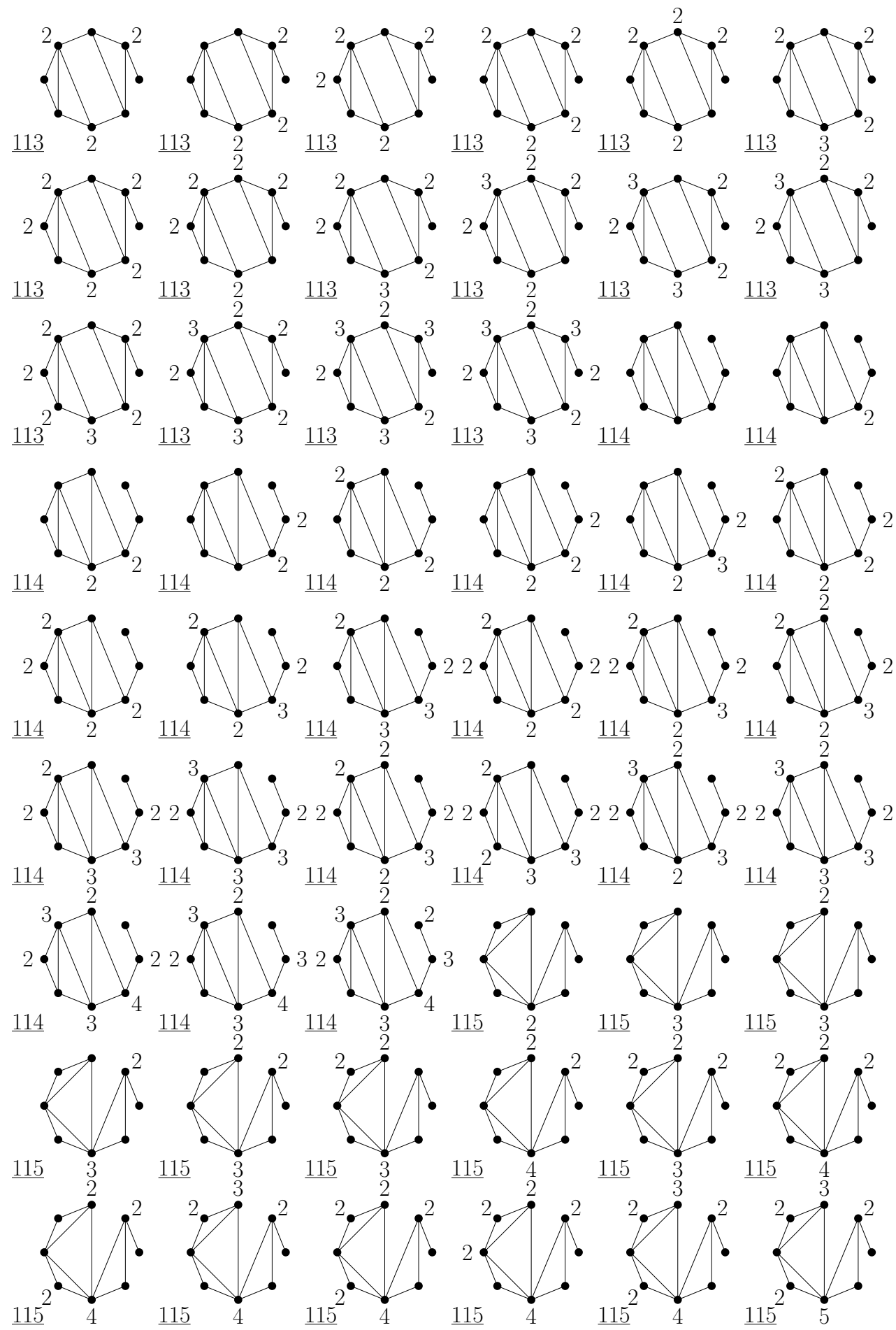

Figure B.47: The set $\mathcal{W}\left(E_{8}\right)$ (continued). 

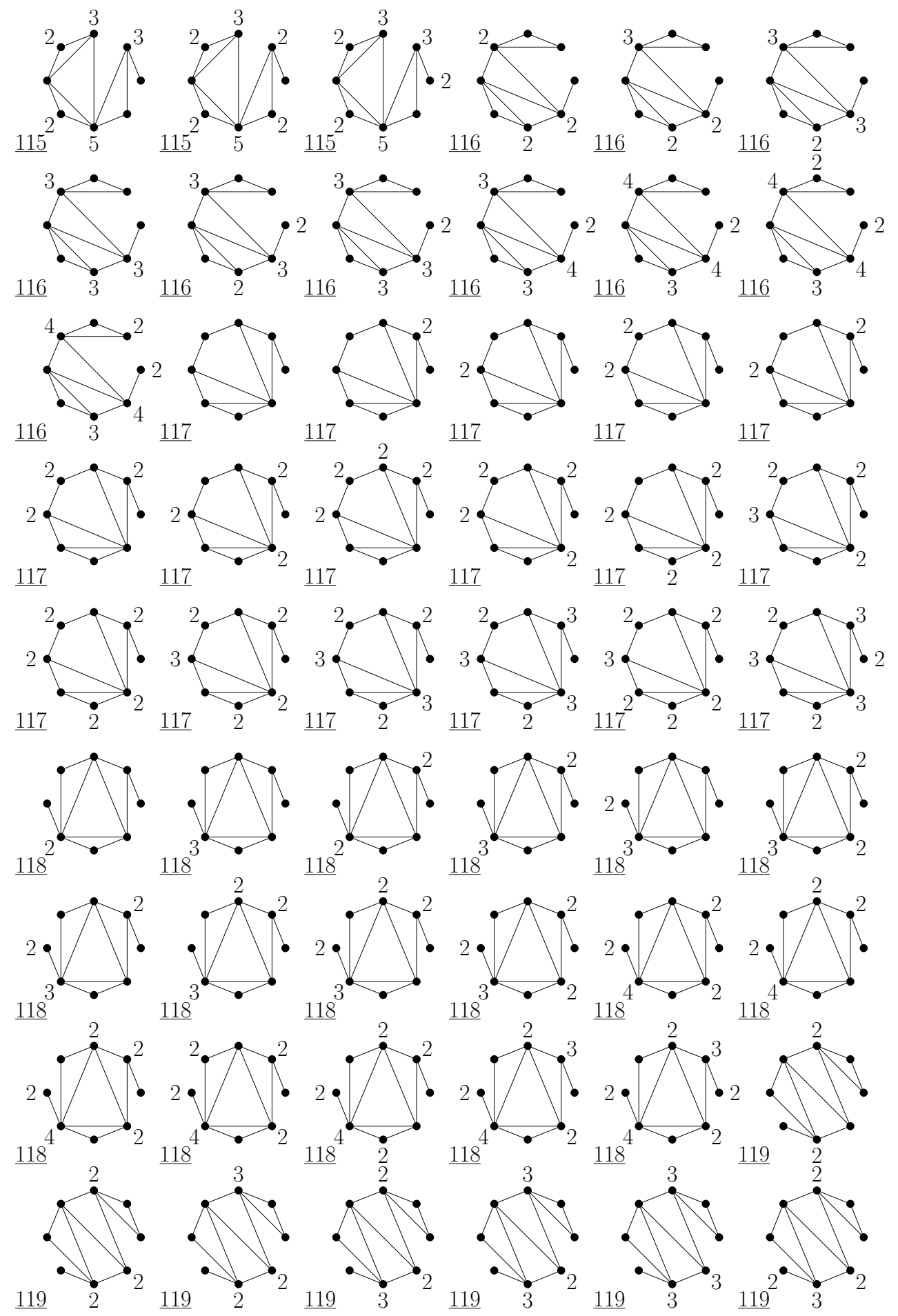

Figure B.48: The set $\mathcal{W}\left(E_{8}\right)$ (continued). 

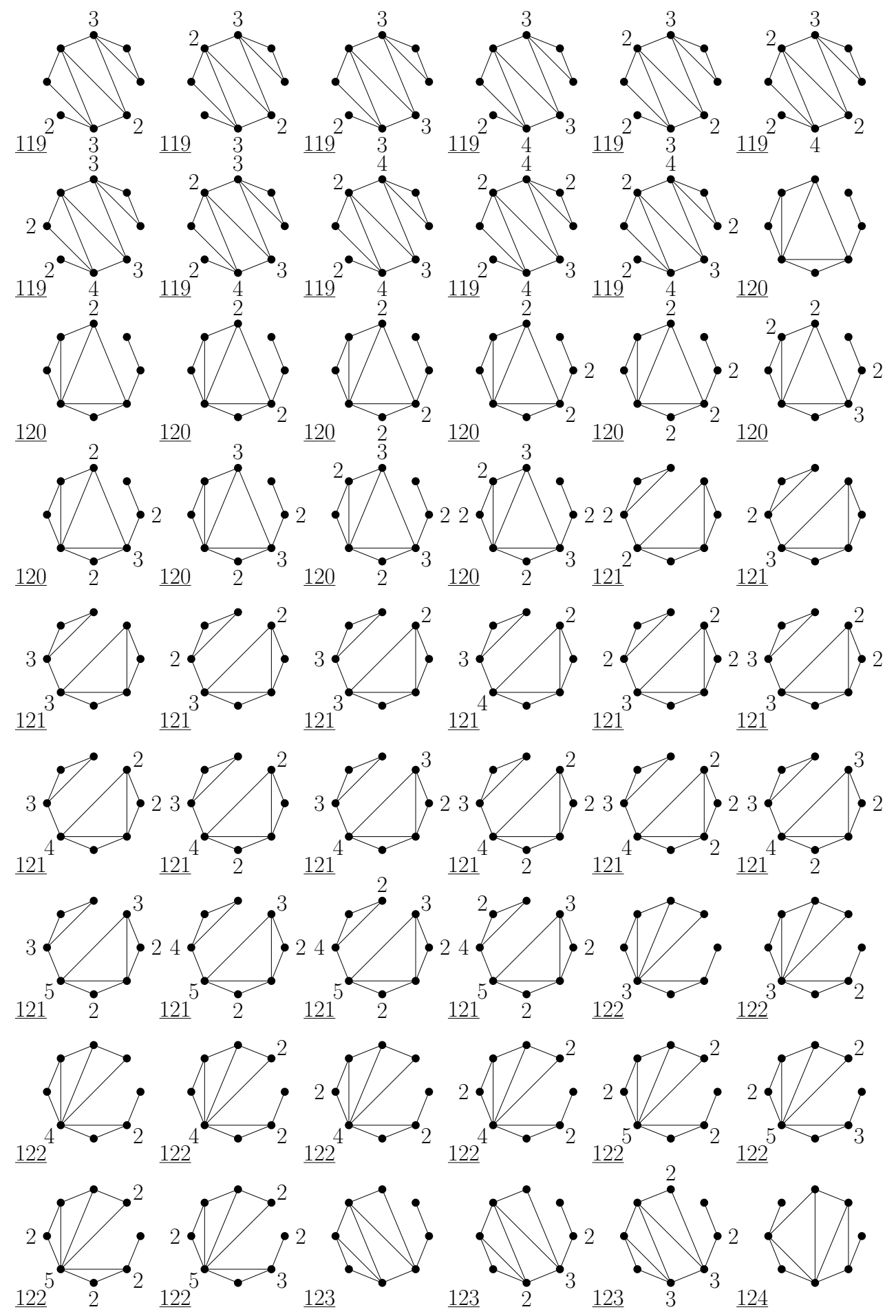

Figure B.49: The set $\mathcal{W}\left(E_{8}\right)$ (continued). 


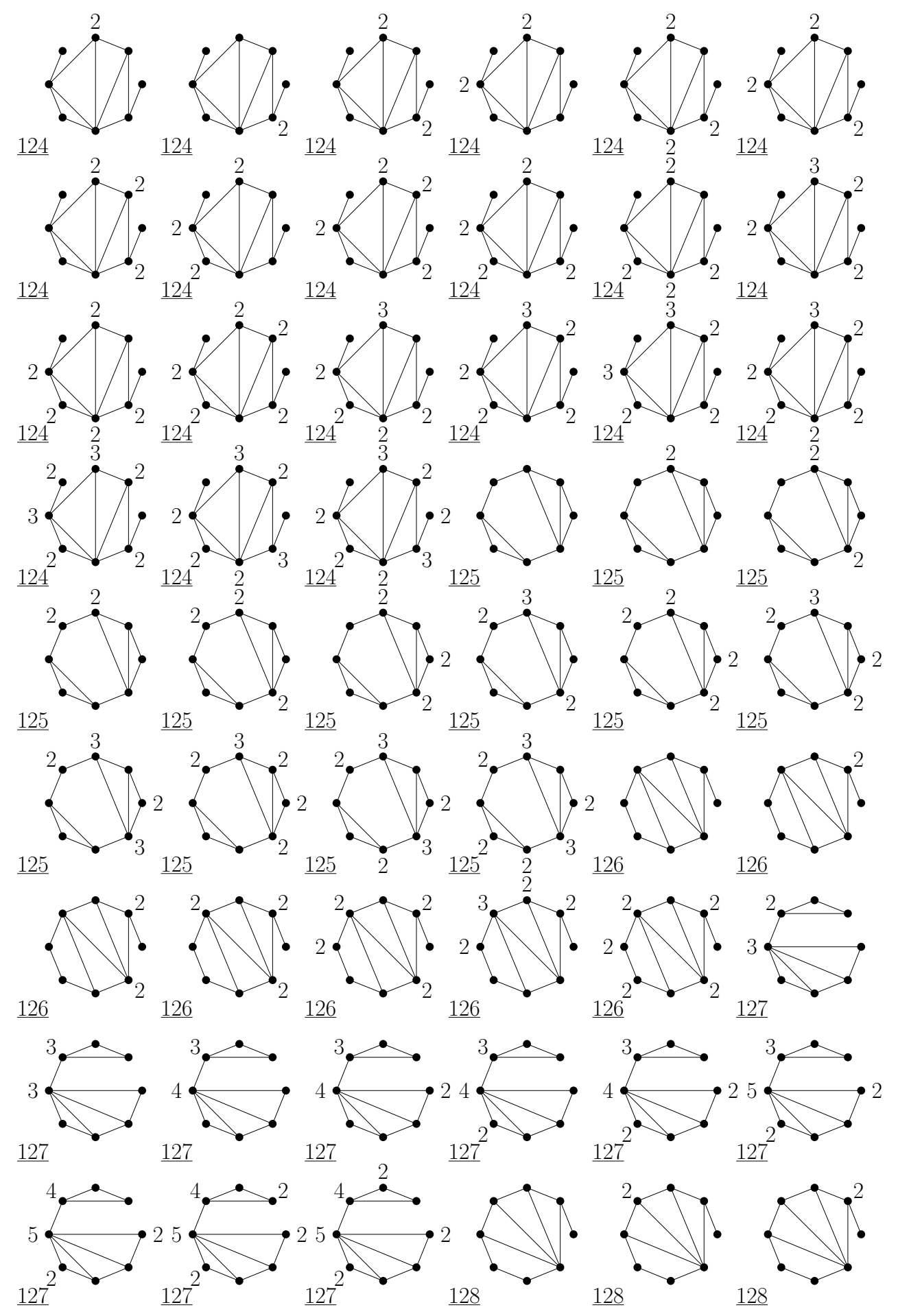

Figure B.50: The set $\mathcal{W}\left(E_{8}\right)$ (continued). 

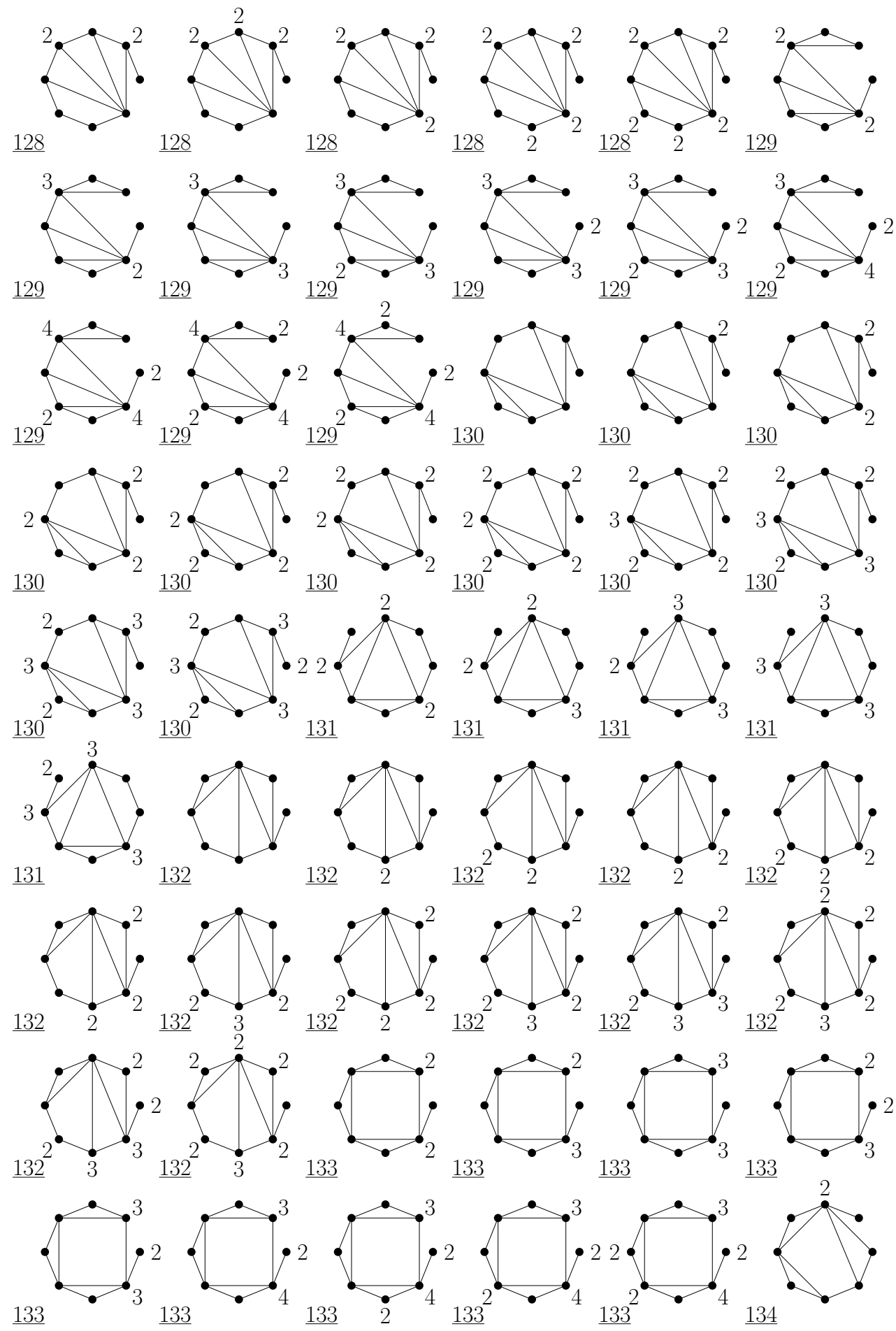

Figure B.51: The set $\mathcal{W}\left(E_{8}\right)$ (continued). 

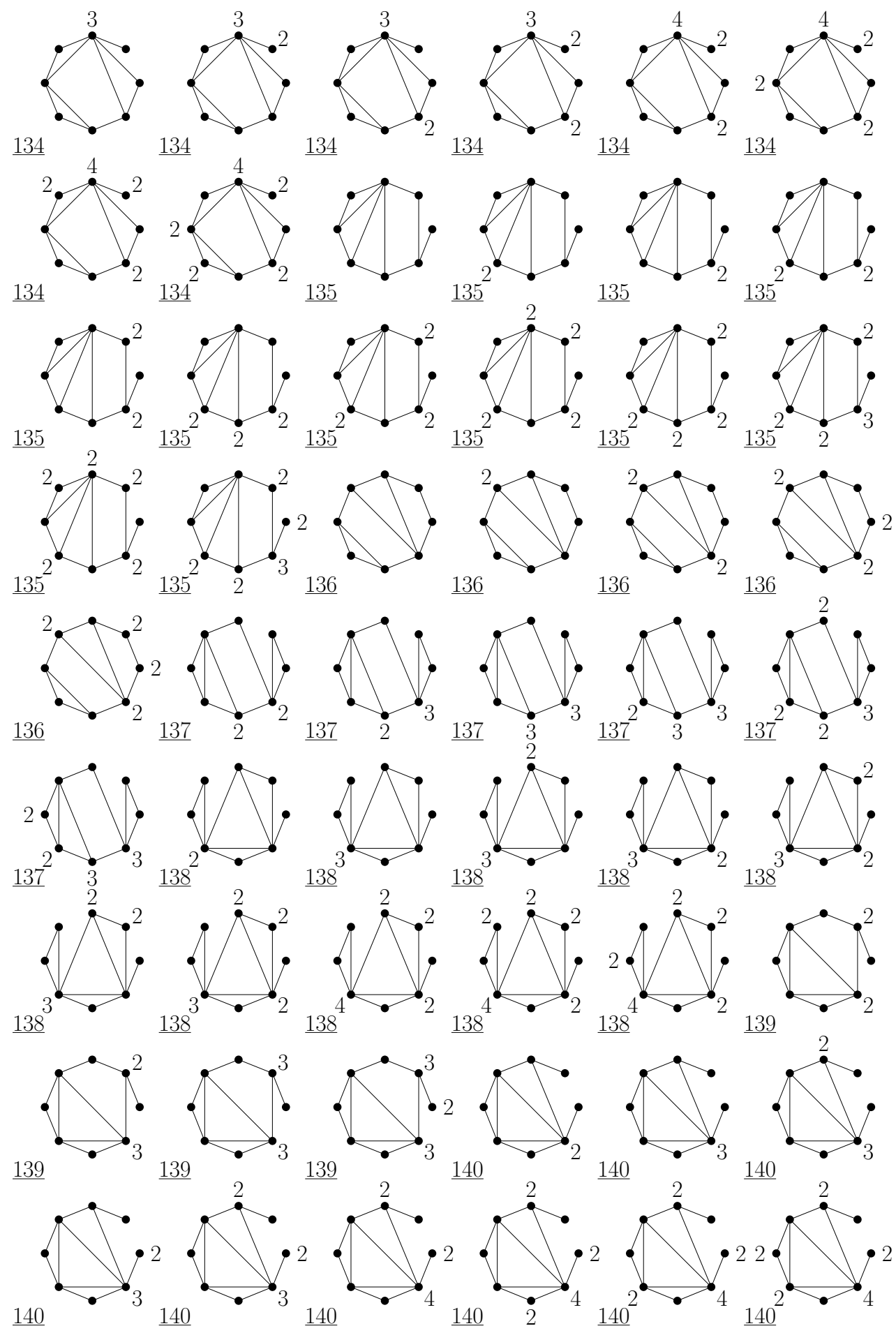

Figure B.52: The set $\mathcal{W}\left(E_{8}\right)$ (continued). 

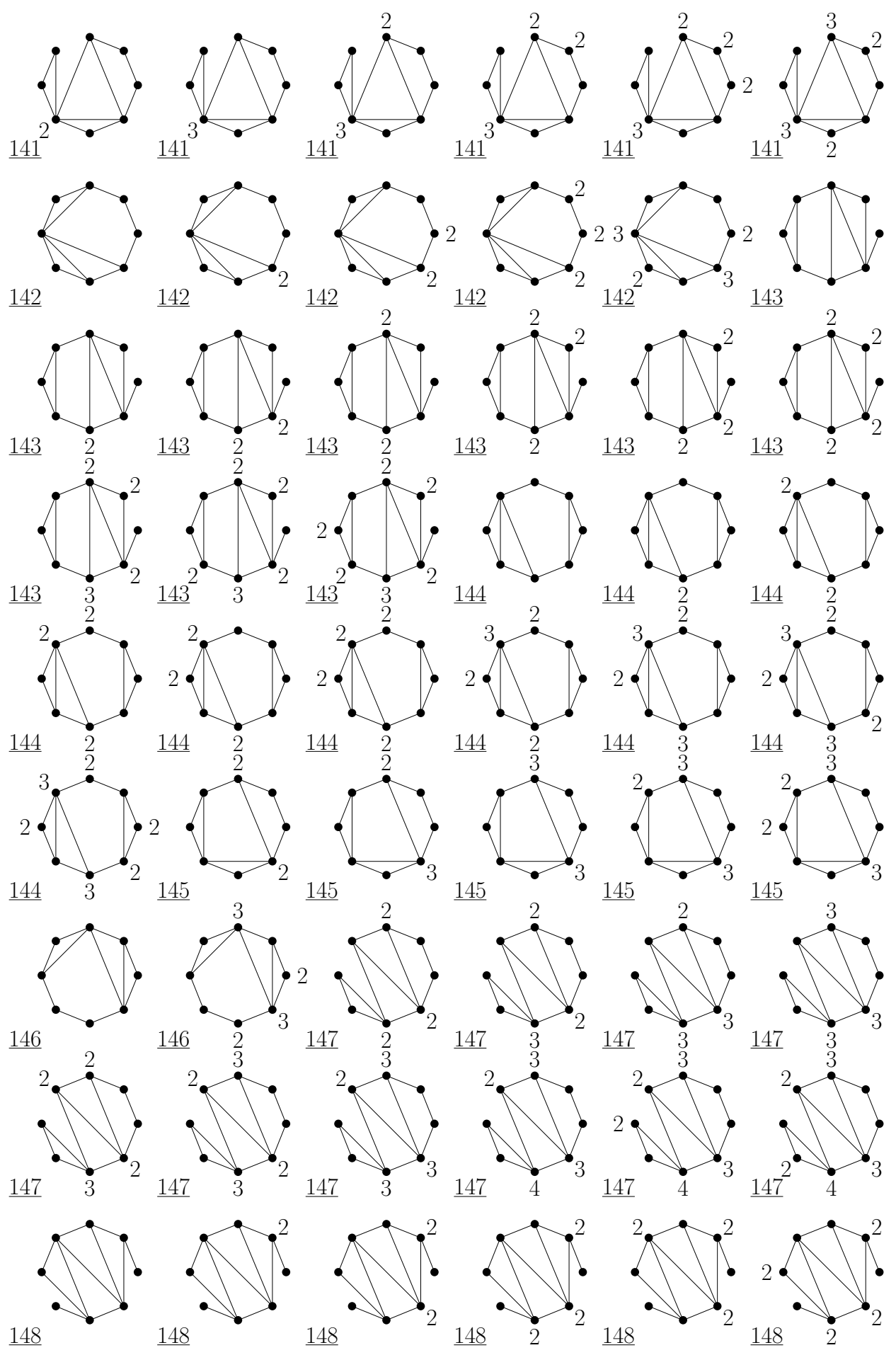

Figure B.53: The set $\mathcal{W}\left(E_{8}\right)$ (continued). 

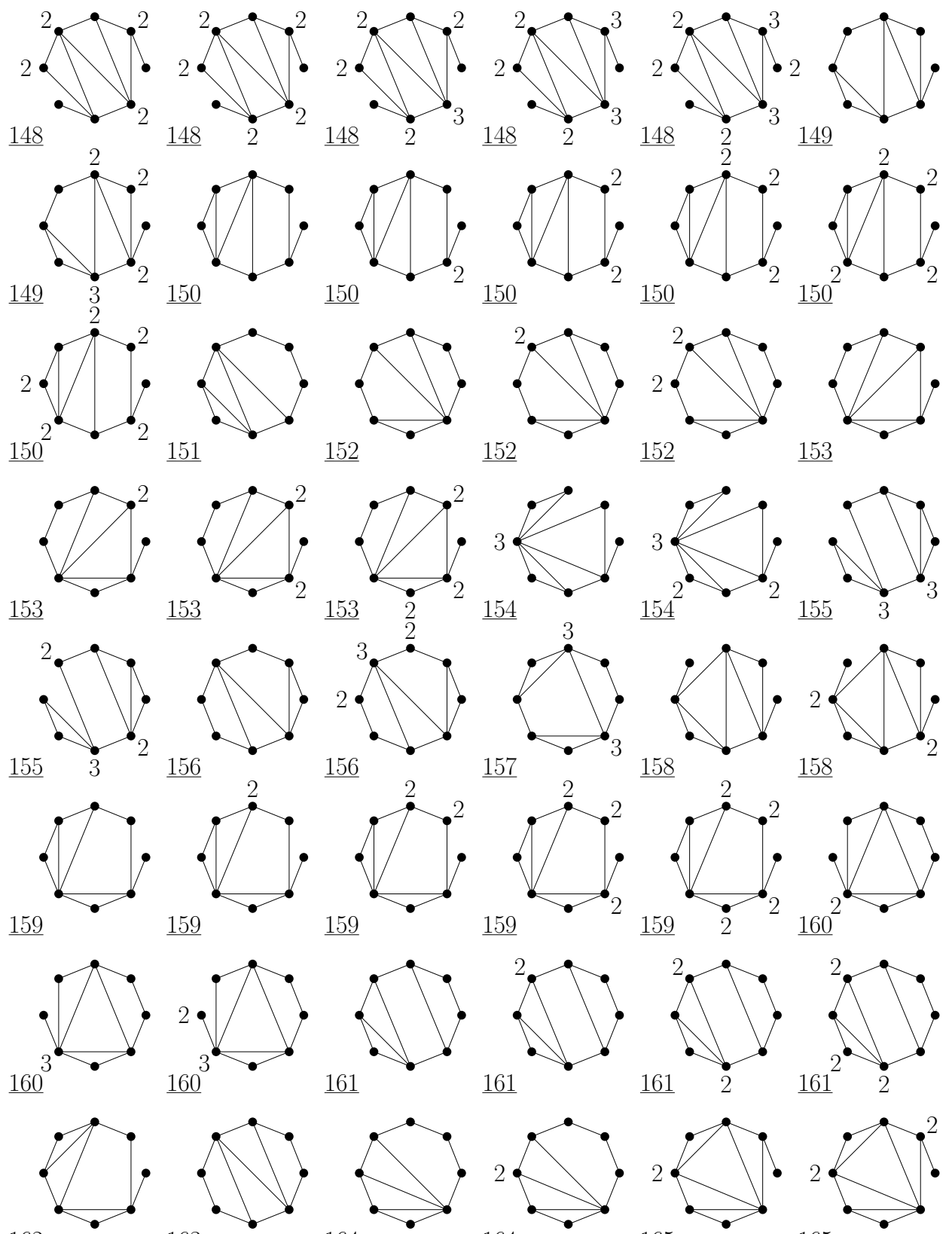

$\underline{162}$
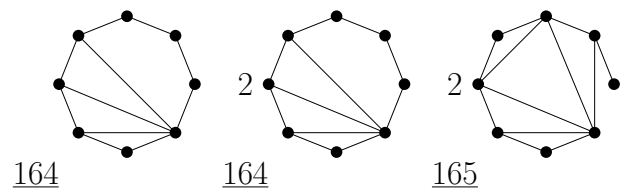

165
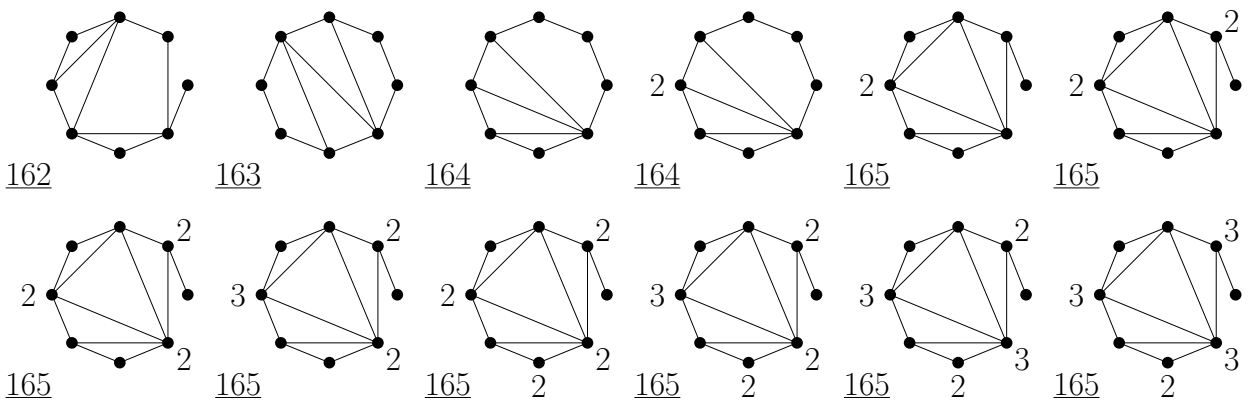

$\underline{165}$ 165

Figure B.54: The set $\mathcal{W}\left(E_{8}\right)$ (continued). 


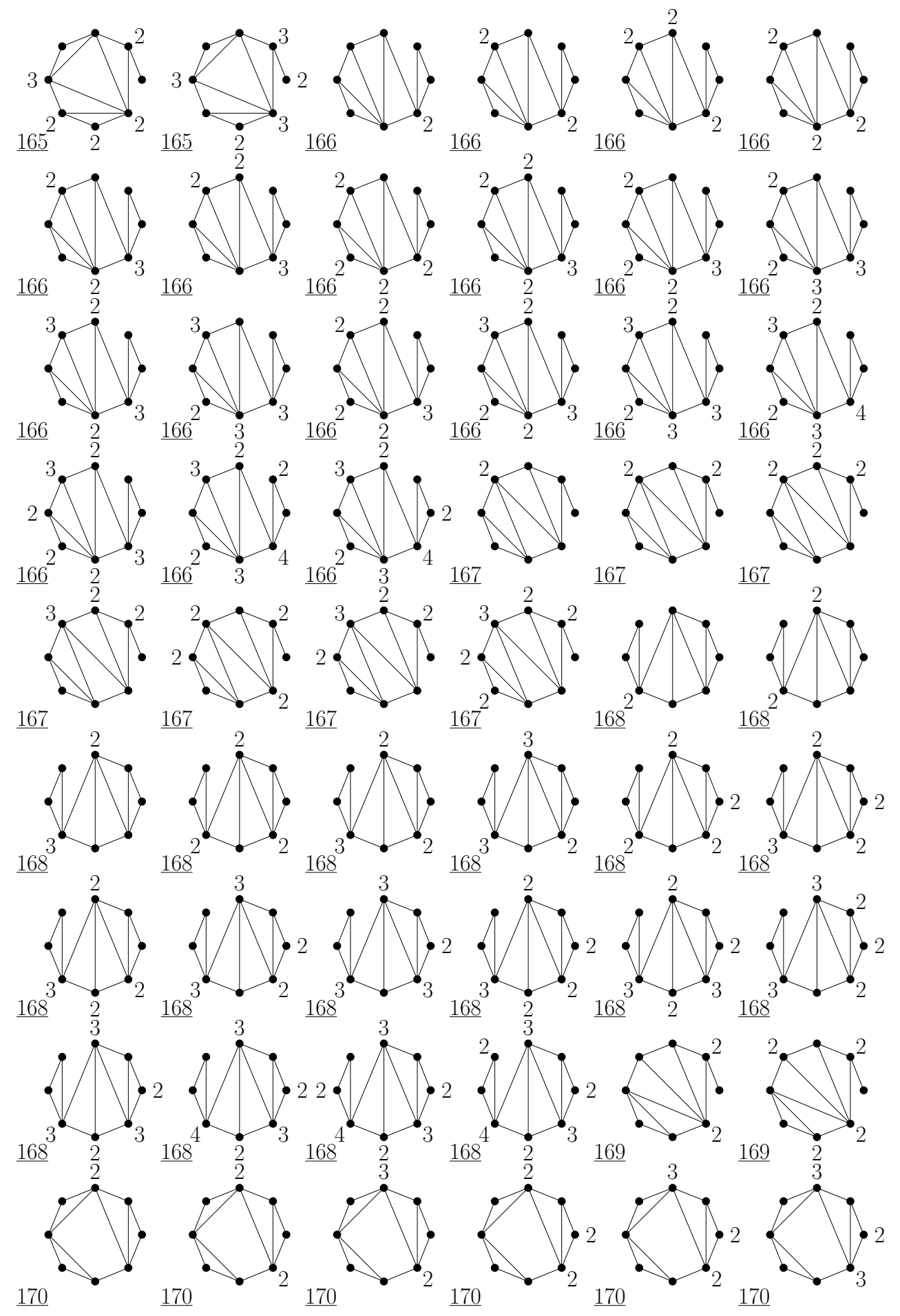

Figure B.55: The set $\mathcal{W}\left(E_{8}\right)$ (continued). 

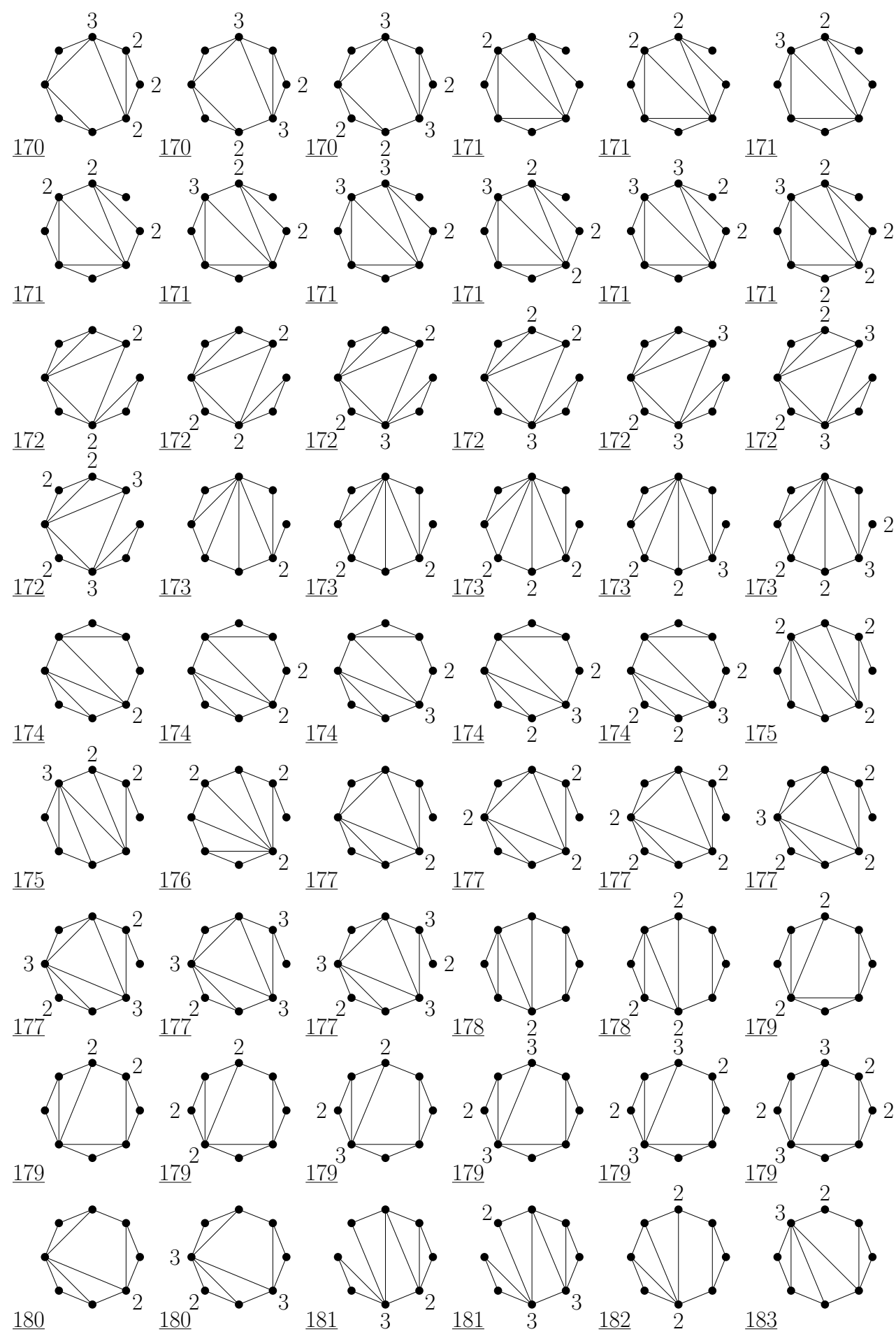

Figure B.56: The set $\mathcal{W}\left(E_{8}\right)$ (continued). 

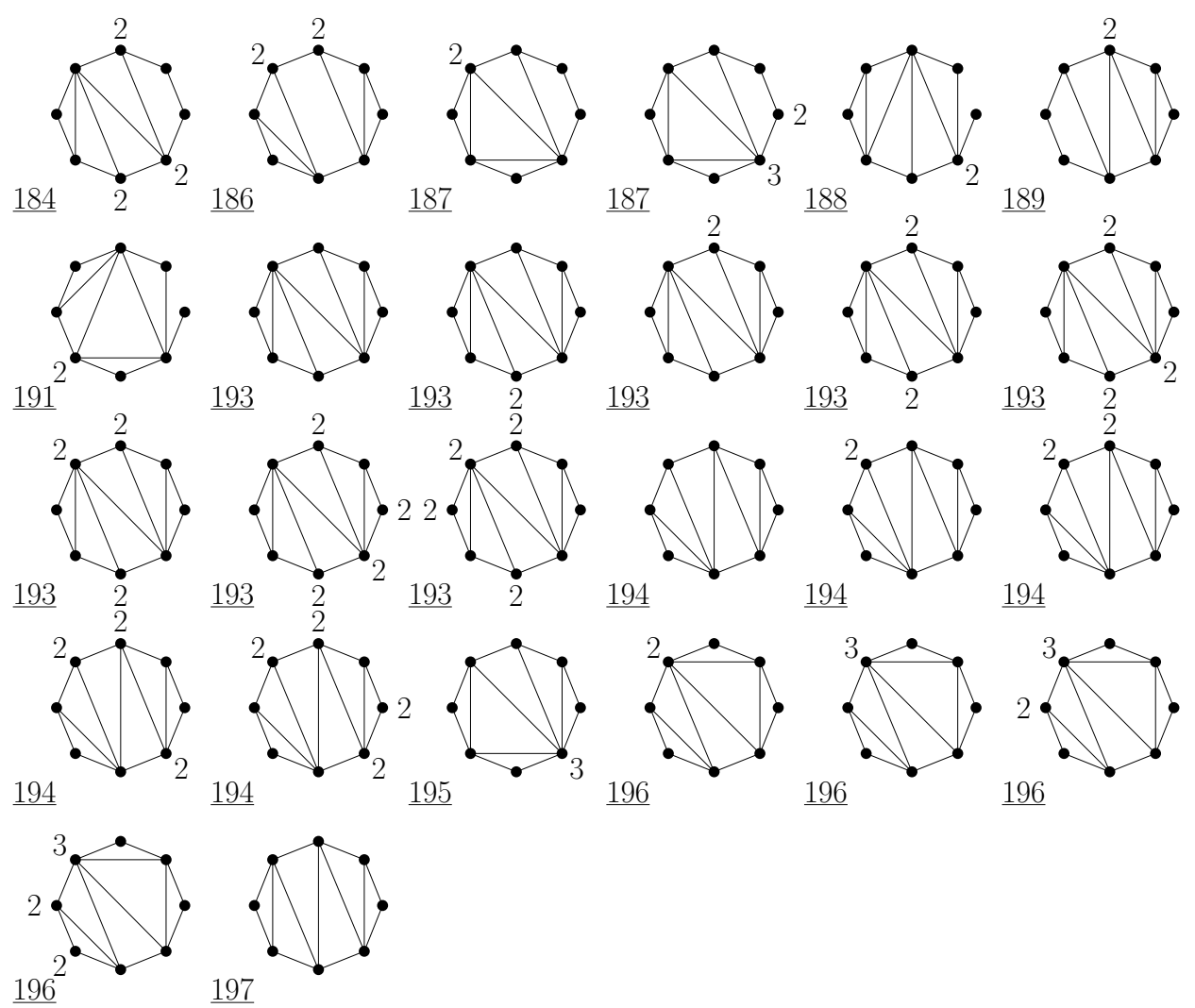

Figure B.57: The set $\mathcal{W}\left(E_{8}\right)$ (continued). 